\title{
Positive Solutions and Iterative Approximations for a Nonlinear Two-Dimensional Difference System with Multiple Delays
}

\author{
Zeqing Liu, ${ }^{1}$ Shin Min Kang, ${ }^{2}$ and Young Chel Kwun ${ }^{3}$ \\ ${ }^{1}$ Department of Mathematics, Liaoning Normal University, Dalian, Liaoning 116029, China \\ ${ }^{2}$ Department of Mathematics and RINS, Gyeongsang National University, Jinju 660-701, Republic of Korea \\ ${ }^{3}$ Department of Mathematics, Dong-A University, Busan 614-714, Republic of Korea
}

Correspondence should be addressed to Young Chel Kwun, yckwun@dau.ac.kr

Received 16 March 2012; Accepted 30 May 2012

Academic Editor: Josef Diblík

Copyright (c) 2012 Zeqing Liu et al. This is an open access article distributed under the Creative Commons Attribution License, which permits unrestricted use, distribution, and reproduction in any medium, provided the original work is properly cited.

This paper studies the nonlinear two-dimensional difference system with multiple delays $\Delta\left(x_{n}+p_{1 n} x_{n-\tau_{1}}\right)+f_{1}\left(n, x_{a_{1 n}}, \ldots, x_{a_{h n}}, y_{b_{1 n}}, \ldots, y_{b_{k n}}\right)=q_{1 n}, \Delta\left(y_{n}+p_{2 n} y_{n-\tau_{2}}\right)+f_{2}\left(n, x_{c_{1 n}}, \ldots, x_{c_{h n}}\right.$, $\left.y_{d_{1 n}}, \ldots, y_{d_{k n}}\right)=q_{2 n}, n \geq n_{0}$. Using the Banach fixed point theorem and a few new analysis techniques, we show the existence of uncountably many bounded positive solutions for the system, suggest Mann iterative algorithms with errors, and discuss the error estimates between the positive solutions and iterative sequences generated by the Mann iterative algorithms. Examples to illustrates the results are included.

\section{Introduction and Preliminaries}

In recent years, there has been an increasing interest in the study of oscillation, nonoscillation, asymptotic behavior, existence and multiplicity of solutions, positive solutions, nonoscillatory solutions, and periodic solutions, respectively, for various difference equations and systems, see for example, [1-34] and the references cited therein.

Jiang and Tang [18] and Graef and Thandapani [14] studied the oscillation of the linear two-dimensional difference system

$$
\begin{gathered}
\Delta x_{n}=p_{n} y_{n}, \\
\Delta y_{n-1}=-q_{n} x_{n}, \quad n \geq n_{0},
\end{gathered}
$$


and nonlinear two-dimensional difference system

$$
\begin{gathered}
\Delta x_{n}=b_{n} g\left(y_{n}\right), \\
\Delta y_{n-1}=-a_{n} f\left(x_{n}\right), \quad n \geq n_{0},
\end{gathered}
$$

where $n_{0} \in \mathbb{N},\left\{p_{n}\right\}_{n \geq n_{0}},\left\{q_{n}\right\}_{n \geq n_{0}},\left\{a_{n}\right\}_{n \geq n_{0}}$, and $\left\{b_{n}\right\}_{n \geq n_{0}}$ are nonnegative sequences, $f, g \in$ $C(\mathbb{R}, \mathbb{R})$ with $u f(u)>0$ and $u g(u)>0$ for all $u \neq 0$. Jiang and Tang [19] also gave some necessary and sufficient conditions for all solutions of System (1.2) to be oscillatory. Agarwal et al. [2] discussed the two-dimensional nonlinear difference system of the form

$$
\begin{gathered}
\Delta x_{n}=a_{n} f\left(y_{n}\right), \\
\Delta y_{n-1}=b_{n} g\left(x_{n}\right), \quad n \geq n_{0},
\end{gathered}
$$

where $n_{0} \in \mathbb{N},\left\{a_{n}\right\}_{n \geq n_{0}}$ and $\left\{b_{n}\right\}_{n \geq n_{0}}$ are positive sequences, $f, g \in C(\mathbb{R}, \mathbb{R})$ are increasing with $u f(u)>0$ and $u g(u)>0$ for all $u \neq 0$, and they provided a classification scheme of positive solutions for System (1.3) and established conditions for the existence of solutions with designated asymptotic behavior. $\mathrm{Li}$ [21] introduced the two-dimensional nonlinear difference system:

$$
\begin{gathered}
\Delta x_{n}=a_{n} f\left(y_{n}\right), \\
\Delta y_{n}=-b_{n} g\left(x_{n}\right), \quad n \geq n_{0},
\end{gathered}
$$

where $n_{0} \in \mathbb{N},\left\{a_{n}\right\}_{n>n_{0}}$ and $\left\{b_{n}\right\}_{n \geq n_{0}}$ are nonnegative sequences, $f, g \in C(\mathbb{R}, \mathbb{R})$ with $u f(u)>0$, and $u g(u)>0$ for all $u \neq 0$, he showed both classification schemes for nonoscillatory solutions of System (1.4) and gave necessary and sufficient conditions for the existence of these solutions. Huo and $\mathrm{Li}[15,16]$ considered the nonlinear two-dimensional difference system

$$
\begin{gathered}
\Delta x_{n}=b_{n} g\left(y_{n}\right), \\
\Delta y_{n}=-a_{n} f\left(x_{n}\right)+r_{n}, \quad n \geq n_{0},
\end{gathered}
$$

and the Emden-Fowler difference system

$$
\begin{gathered}
\Delta x_{n}=b_{n} g\left(y_{n}\right), \\
\Delta y_{n-1}=-a_{n} f\left(x_{n}\right)+r_{n}, \quad n \geq 1,
\end{gathered}
$$

where $n_{0} \in \mathbb{N},\left\{a_{n}\right\}_{n \geq n_{0}}$ is a real sequence, $\left\{b_{n}\right\}_{n \geq n_{0}}$ is a nonnegative sequence, $\sum_{i=1}^{\infty}\left|r_{i}\right|<+\infty$, $f, g \in C(\mathbb{R}, \mathbb{R})$ with $u f(u)>0$, and $u g(u)>0$ for all $u \neq 0$, they proved some oscillation results for Systems (1.5) and (1.6). Jiang and Li [17] investigated the nonlinear two-dimensional difference system:

$$
\begin{gathered}
\Delta x_{n}=a_{n} g\left(y_{n}\right), \\
\Delta y_{n-1}=f\left(n, x_{n}\right), \quad n \geq n_{0},
\end{gathered}
$$


where $n_{0} \in \mathbb{N},\left\{a_{n}\right\}_{n \geq n_{0}}$ is a nonnegative sequence, $f \in C\left(\mathbb{N}_{n_{0}} \times \mathbb{R}, \mathbb{R}\right), g \in C(\mathbb{R}, \mathbb{R})$ with $u f(n, u)>0$ for all $n \in \mathbb{N}_{n_{0}}$, and $u \neq 0$ and $u g(u)>0$ for all $u \neq 0$, they obtained some necessary and sufficient conditions for all solutions of System (1.7) to be oscillatory. Thandapani and Kumar [31] studied the oscillation for the nonlinear two-dimensional difference system of the neutral type

$$
\begin{gathered}
\Delta\left(x_{n}-a_{n} x_{\sigma_{n}}\right)=p_{n} g\left(y_{n}\right), \\
\Delta y_{n}=\delta q_{n} f\left(x_{\tau_{n}}\right), \quad n \geq n_{0},
\end{gathered}
$$

where $\delta= \pm 1, n_{0} \in \mathbb{N},\left\{a_{n}\right\}_{n \geq n_{0}}$ is a positive sequence, $\left\{p_{n}\right\}_{n \geq n_{0}}$ and $\left\{q_{n}\right\}_{n \geq n_{0}}$ are nonnegative sequences with $\sum_{i=n_{0}}^{\infty} p_{i}=+\infty, \lim _{n \rightarrow \infty} \sigma_{n}=\lim _{n \rightarrow \infty} \tau_{n}=+\infty, f, g \in C(\mathbb{R}, \mathbb{R})$ are nondecreasing with $u f(u)>0$ and $u g(u)>0$ for all $u \neq 0$. Wu and Liu [33] established the existence and multiplicity of periodic solutions for the first-order neutral difference system

$$
\begin{aligned}
& \Delta\left(x_{n}-c x_{n-\tau}\right)=a_{1 n} g_{1}\left(x_{n}\right) x_{n}-\lambda b_{1 n} f_{1}\left(x_{n-\tau_{1 n}}, y_{n-\rho_{1 n}}\right), \\
& \Delta\left(y_{n}-c y_{n-\tau}\right)=a_{2 n} g_{2}\left(y_{n}\right) y_{n}-\mu b_{2 n} f_{2}\left(x_{n-\tau_{2 n}}, y_{n-\rho_{2 n}}\right),
\end{aligned}
$$

where $\tau \in \mathbb{N}, \lambda, \mu \in \mathbb{R}^{+} \backslash\{0\}, c \in \mathbb{R}$ with $|c| \neq 1,\left\{a_{1 n}\right\},\left\{a_{2 n}\right\},\left\{b_{1 n}\right\}$, and $\left\{b_{2 n}\right\}$ are positive $T$-periodic sequences, $\left\{\tau_{1 n}\right\},\left\{\tau_{2 n}\right\},\left\{\rho_{1 n}\right\}$, and $\left\{\rho_{2 n}\right\}$ are positive $T$-periodic integer sequences. Tang [30] proved the existence results of a bounded nonoscillatory solution for the secondorder linear delay difference equation

$$
\Delta^{2} x_{n}=p_{n} x_{n-k}, \quad n \geq 0,
$$

where $k \in \mathbb{N}$ and $\left\{p_{n}\right\}_{n \geq 0}$ is a nonnegative sequence. Cheng [20] utilized the Banach fixed point theorem to discuss the existence of a nonoscillatory solution for the second-order neutral delay difference equation with positive and negative coefficients:

$$
\Delta^{2}\left(x_{n}+p x_{n-m}\right)+p_{n} x_{n-k}-q_{n} x_{n-l}=0, \quad n \geq n_{0},
$$

where $m, k, l \in \mathbb{N}, p \in \mathbb{R} \backslash\{-1\},\left\{p_{n}\right\}_{n \geq 0}$ and $\left\{q_{n}\right\}_{n \geq 0}$ are nonnegative sequences with $\sum_{n=n_{0}}^{\infty} n \max \left\{p_{n}, q_{n}\right\}<+\infty$. M. Migda and J. Migda [29] obtained the asymptotic behavior of the second-order neutral difference equation:

$$
\Delta^{2}\left(x_{n}+p x_{n-k}\right)+f\left(n, x_{n}\right)=0, \quad n \geq 1,
$$

where $p \in \mathbb{R}, k \in \mathbb{N}_{0}$, and $f \in C(\mathbb{N} \times \mathbb{R}, \mathbb{R})$. Liu et al. [27] studied the global existence of uncountably many bounded nonoscillatory solutions for the second-order nonlinear neutral delay difference equation:

$$
\Delta\left(a_{n} \Delta\left(x_{n}+b x_{n-\tau}\right)\right)+f\left(n, x_{n-d_{1 n}}, x_{n-d_{2 n}}, \ldots, x_{n-d_{k n}}\right)=c_{n}, \quad n \geq n_{0},
$$


where $b \in \mathbb{R}, \tau, k \in \mathbb{N}, n_{0} \in \mathbb{N}_{0},\left\{a_{n}\right\}_{n \in \mathbb{N}_{n_{0}}}$ is a positive sequence, $\left\{c_{n}\right\}_{n \in \mathbb{N}_{n_{0}}}$ is a real sequence, $\bigcup_{l=1}^{k}\left\{d_{l n}\right\}_{n \in \mathbb{N}_{n_{0}}} \subseteq \mathbb{Z}$ with $\lim _{n \rightarrow \infty}\left(n-d_{l n}\right)=+\infty$ for $l \in\{1,2, \ldots, k\}$, and $f: \mathbb{N}_{n_{0}} \times \mathbb{R}^{k} \rightarrow \mathbb{R}$ is a mapping.

The purpose of this paper is to study the below nonlinear two-dimensional difference system with multiple delays

$$
\begin{gathered}
\Delta\left(x_{n}+p_{1 n} x_{n-\tau_{1}}\right)+f_{1}\left(n, x_{a_{1 n}}, \ldots, x_{a_{h n}}, y_{b_{1 n}}, \ldots, y_{b_{k n}}\right)=q_{1 n}, \\
\Delta\left(y_{n}+p_{2 n} y_{n-\tau_{2}}\right)+f_{2}\left(n, x_{c_{1 n}}, \ldots, x_{c_{h n}}, y_{d_{1 n}}, \ldots, y_{d_{k n}}\right)=q_{2 n}, \quad n \geq n_{0},
\end{gathered}
$$

where $h, k, \tau_{i} \in \mathbb{N}, n_{0} \in \mathbb{N}_{0},\left\{p_{i n}\right\}_{n \in \mathbb{N}_{n_{0}}},\left\{q_{i n}\right\}_{n \in \mathbb{N}_{n_{0}}} \subset \mathbb{R}$, and $f_{i} \in C\left(\mathbb{N}_{n_{0}} \times \mathbb{R}^{h+k}, \mathbb{R}\right)$ for $i \in \Lambda_{2}$, $\left\{a_{l n}\right\}_{n \in \mathbb{N}_{n_{0}}}\left\{c_{l n}\right\}_{n \in \mathbb{N}_{n_{0}}} \subset \mathbb{Z}$ with $\lim _{n \rightarrow \infty} a_{l n}=\lim _{n \rightarrow \infty} c_{l n}=+\infty$ for $l \in \Lambda_{h}$ and $\left\{b_{j n}\right\}_{n \in \mathbb{N}_{n_{0}}}$ $\left\{d_{j n}\right\}_{n \in \mathbb{N}_{n_{0}}} \subset \mathbb{Z}$ with $\lim _{n \rightarrow \infty} b_{j n}=\lim _{n \rightarrow \infty} d_{j n}=+\infty$ for $j \in \Lambda_{k}$. It is easy to see that the system (1.14) includes Systems (1.1)-(1.9), (1.10)-(1.13), and a lot of the first- and second-order nonlinear, half-linear, and quasilinear difference equations as special cases. Using the Banach fixed point theorem and some analysis techniques, we prove the existence of uncountably many bounded positive solutions for System (1.14), establish their iterative approximations, and discuss the error estimates between the positive solutions and iterative approximations. Our results sharp, and improve [14, Theorem 1] and [27, Theorems 2.1-2.7]. To illustrate our results, fifteen examples are also included.

Throughout this paper, we assume that $\Delta$ is the forward difference operator defined by $\Delta x_{n}=x_{n+1}-x_{n}, \mathbb{R}=(-\infty,+\infty), \mathbb{R}^{+}=[0,+\infty), \mathbb{Z}, \mathbb{N}$, and $\mathbb{N}_{0}$ stand for the sets of all integers, positive integers, and nonnegative integers, respectively,

$$
\begin{gathered}
\mathbb{N}_{n_{0}}=\left\{n: n \in \mathbb{N}_{0} \text { with } n \geq n_{0}\right\}, \quad \Lambda_{n}=\{1, \ldots, n\}, \quad \forall n \in \mathbb{N}, \\
\alpha=\inf \left\{n_{0}-\tau_{1}, n_{0}-\tau_{2}, a_{l n}, c_{l n}, b_{j n}, d_{j n}: n \in \mathbb{N}_{n_{0}}, l \in \Lambda_{h}, j \in \Lambda_{k}\right\}, \\
\mathbb{Z}_{\alpha}=\{n: n \in \mathbb{Z} \text { with } n \geq \alpha\},
\end{gathered}
$$

$l_{\alpha}^{\infty}$ denotes the Banach space of all bounded sequences on $\mathbb{Z}_{\alpha}$ with norm

$$
\|x\|=\sup _{n \in \mathbb{Z}_{\alpha}}\left|x_{n}\right| \quad \text { for } x=\left\{x_{n}\right\}_{n \in \mathbb{Z}_{\alpha}} \in l_{\alpha}^{\infty} .
$$

Let

$A(N, M)$

$$
=\left\{(x, y)=\left(\left\{x_{n}\right\}_{n \in \mathbb{Z}_{\alpha}}\left\{y_{n}\right\}_{n \in \mathbb{Z}_{\alpha}}\right) \in l_{\alpha}^{\infty} \times l_{\alpha}^{\infty}: N \leq x_{n} \leq M, N \leq y_{n} \leq M, n \in \mathbb{Z}_{\alpha}\right\},
$$

$A\left(N, M, N_{0}, M_{0}\right)$

$$
=\left\{(x, y)=\left(\left\{x_{n}\right\}_{n \in \mathbb{Z}_{\alpha}}\left\{y_{n}\right\}_{n \in \mathbb{Z}_{\alpha}}\right) \in l_{\alpha}^{\infty} \times l_{\alpha}^{\infty}: N \leq x_{n} \leq M, N_{0} \leq y_{n} \leq M_{0}, n \in \mathbb{Z}_{\alpha}\right\},
$$


for any $M>N>0$ and $M_{0}>N_{0}>0$. It is easy to see that $A(N, M)$ and $A\left(N, M, N_{0}, M_{0}\right)$ are bounded closed and convex subsets of the Banach space $l_{\alpha}^{\infty} \times l_{\alpha}^{\infty}$ with norm

$$
\|(x, y)\|_{1}=\max \{\|x\|,\|y\|\} \quad \text { for }(x, y) \in l_{\alpha}^{\infty} \times l_{\alpha}^{\infty} \text {. }
$$

By a solution of System (1.14), we mean a sequence $\left(\left\{x_{n}\right\}_{n \in \mathbb{Z}_{\alpha}},\left\{y_{n}\right\}_{n \in \mathbb{Z}_{\alpha}}\right)$ with a positive integer $T \geq n_{0}+\tau_{1}+\tau_{2}+|\alpha|$ such that System (1.14) is satisfied for all $n \geq T$. A solution $\left(\left\{x_{n}\right\}_{n \in \mathbb{Z}_{\alpha}},\left\{y_{n}\right\}_{n \in \mathbb{Z}_{\alpha}}\right.$ ) of System (1.14) is said to be positive if both components are positive.

Lemma 1.1 (see [35]). Let $\left\{A_{n}\right\}_{n \geq 0},\left\{B_{n}\right\}_{n \geq 0},\left\{C_{n}\right\}_{n \geq 0}$, and $\left\{D_{n}\right\}_{n \geq 0}$ be four nonnegative real sequences satisfying the inequality

$$
A_{n+1} \leq\left(1-D_{n}\right) A_{n}+D_{n} B_{n}+C_{n}, \quad \forall n \geq 0,
$$

where $\left\{D_{n}\right\}_{n \geq 0} \subset[0,1], \sum_{n=0}^{\infty} D_{n}=+\infty, \lim _{n \rightarrow \infty} B_{n}=0$, and $\sum_{n=0}^{\infty} C_{n}<+\infty$. Then $\lim _{n \rightarrow \infty} A_{n}=$ 0 .

Lemma 1.2 (see [36]). Let $\tau \in \mathbb{N}, n_{0} \in \mathbb{N}_{0}$, and $\left\{B_{n}\right\}_{n \in \mathbb{N}_{n_{0}}}$ be a nonnegative sequence. Then

$$
\sum_{i=0}^{\infty} \sum_{n=n_{0}+i \tau}^{\infty} B_{n}<+\infty \Longleftrightarrow \sum_{n=n_{0}}^{\infty} n B_{n}<+\infty
$$

\section{Main Results}

In this section, we investigate the existence of uncountably many bounded positive solutions for System (1.14) and their iterative approximations and the error estimates between the positive solutions and iterative approximations.

Theorem 2.1. Assume that there exist constants $M, N \in \mathbb{R}^{+} \backslash\{0\}, n_{1} \in \mathbb{N}_{n_{0}}$, and four nonnegative sequences $\left\{r_{1 n}\right\}_{n \in \mathbb{N}_{n_{0}}},\left\{r_{2 n}\right\}_{n \in \mathbb{N}_{n_{0}}},\left\{t_{1 n}\right\}_{n \in \mathbb{N}_{n_{0}}}$ and $\left\{t_{2 n}\right\}_{n \in \mathbb{N}_{n_{0}}}$ satisfying

$$
\begin{gathered}
\left|f_{i}\left(n, u_{1}, \ldots, u_{h}, v_{1}, \ldots, v_{k}\right)\right| \leq r_{i n}, \quad \forall\left(n, u_{l}, v_{j}\right) \in \mathbb{N}_{n_{1}} \times[N, M]^{2}, l \in \Lambda_{h}, j \in \Lambda_{k}, i \in \Lambda_{2} \\
\left|f_{i}\left(n, u_{1}, \ldots, u_{h}, v_{1}, \ldots, v_{k}\right)-f_{i}\left(n, w_{1}, \ldots, w_{h}, z_{1}, \ldots, z_{k}\right)\right| \\
\leq t_{i n} \max \left\{\left|u_{l}-w_{l}\right|,\left|v_{j}-z_{j}\right|: l \in \Lambda_{h}, j \in \Lambda_{k}\right\} \\
\forall\left(n, u_{l}, w_{l}, v_{j}, z_{j}\right) \in \mathbb{N}_{n_{1}} \times[N, M]^{4}, l \in \Lambda_{h}, j \in \Lambda_{k}, i \in \Lambda_{2} \\
\sum_{n=n_{0}}^{\infty} n \max \left\{r_{i n}, t_{i n},\left|q_{i n}\right|: i \in \Lambda_{2}\right\}<+\infty \\
N<M, \quad p_{i n}=-1, \quad \forall n \geq n_{1}, i \in \Lambda_{2} .
\end{gathered}
$$


Then,

(a) for each $L \in(N, M)$, there exist $\theta_{L} \in(0,1)$ and $T_{L} \geq \max \left\{\tau_{1}, \tau_{2}\right\}+n_{1}+|\alpha|$ such that for any $\left(x^{0}, y^{0}\right)=\left(\left\{x_{n}^{0}\right\}_{n \in \mathbb{Z}_{\alpha}},\left\{y_{n}^{0}\right\}_{n \in \mathbb{Z}_{\alpha}}\right) \in A(N, M)$, the Mann iterative sequence with errors $\left\{\left(x^{\mu}, y^{\mu}\right)\right\}_{\mu \in \mathbb{N}_{0}}=\left\{\left(\left\{x_{n}^{\mu}\right\}_{n \in \mathbb{Z}_{\alpha}},\left\{y_{n}^{\mu}\right\}_{n \in \mathbb{Z}_{\alpha}}\right)\right\}_{\mu \in \mathbb{N}_{0}}$ generated by the schemes

$$
\begin{aligned}
& \left\{\begin{array}{l}
\left(1-\beta_{\mu}-\gamma_{\mu}\right) x_{n}^{\mu} \\
+\beta_{\mu}\left\{L-\sum_{l=0}^{\infty} \sum_{m=n+(l+1) \tau_{1}}^{\infty}\left[f_{1}\left(m, x_{a_{1 m}}^{\mu}, \ldots, x_{a_{h m}}^{\mu}, y_{b_{1 m}}^{\mu}, \ldots, y_{b_{k m}}^{\mu}\right)-q_{1 m}\right]\right\}
\end{array}\right. \\
& x_{n}^{\mu+1}= \begin{cases}+\gamma_{\mu} \delta_{1 n^{\prime}}^{\mu} & \mu \geq 0, n \geq T_{L},\end{cases} \\
& \left(1-\beta_{\mu}-\gamma_{\mu}\right) x_{T_{L}}^{\mu} \\
& +\beta_{\mu}\left\{L-\sum_{l=0}^{\infty} \sum_{m=T_{L}+(l+1) \tau_{1}}^{\infty}\left[f_{1}\left(m, x_{a_{1 m}}^{\mu}, \ldots, x_{a_{h m}}^{\mu}, y_{b_{1 m}}^{\mu}, \ldots, y_{b_{k m}}^{\mu}\right)-q_{1 m}\right]\right\} \\
& \left(+\gamma_{\mu} \delta_{1 T_{L}{ }^{\mu}}^{\mu} \quad \mu \geq 0, \alpha \leq n<T_{L},\right.
\end{aligned}
$$

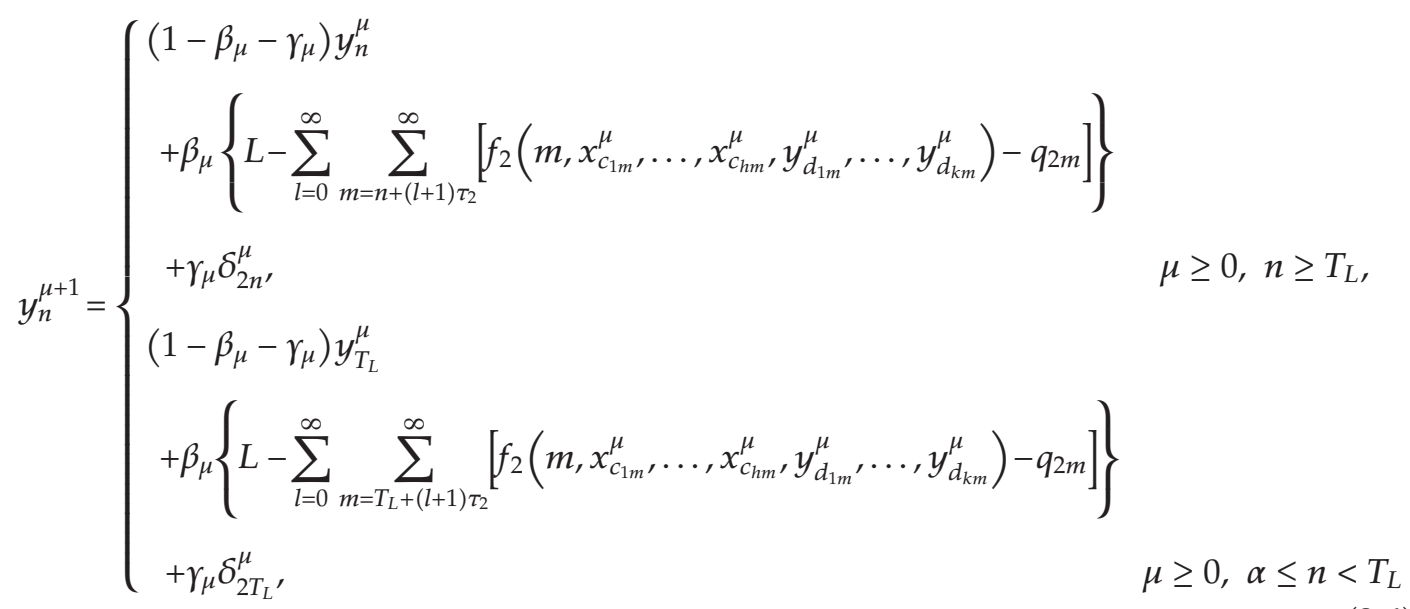

converges to a bounded positive solution $(x, y) \in A(N, M)$ of System (1.14) and has the error estimate:

$$
\left\|\left(x^{\mu+1}, y^{\mu+1}\right)-(x, y)\right\|_{1} \leq\left(1-\beta_{\mu}\left(1-\theta_{L}\right)\right)\left\|\left(x^{\mu}, y^{\mu}\right)-(x, y)\right\|_{1}+2 M \gamma_{\mu}, \quad \forall \mu \geq 0
$$


where $\left\{\beta_{\mu}\right\}_{\mu \in \mathbb{N}_{0}},\left\{\gamma_{\mu}\right\}_{\mu \in \mathbb{N}_{0}} \subset[0,1]$ and $\left\{\left(\delta_{1}^{\mu}, \delta_{2}^{\mu}\right)\right\}_{\mu \in \mathbb{N}_{0}}=\left\{\left(\left\{\delta_{1 n}^{\mu}\right\}_{n \in \mathbb{Z}_{\alpha}}\left\{\delta_{2 n}^{\mu}\right\}_{n \in \mathbb{Z}_{\alpha}}\right)\right\}_{\mu \in \mathbb{N}_{0}} \subset$ $A(N, M)$ are arbitrary sequences with

$$
\begin{gathered}
\qquad \sum_{\mu=0}^{\infty} \beta_{\mu}=+\infty \\
\sum_{\mu=0}^{\infty} \gamma_{\mu}<+\infty \text { or there exists a sequence }\left\{\eta_{\mu}\right\}_{\mu \in \mathbb{N}_{0}} \subset[0,+\infty) \text { satisfying } \\
\gamma_{\mu}=\beta_{\mu} \eta_{\mu}, \quad \forall \mu \in \mathbb{N}_{0}, \quad \lim _{\mu \rightarrow \infty} \eta_{\mu}=0,
\end{gathered}
$$

(b) System (1.14) has uncountably many bounded positive solutions in $A(N, M)$.

Proof. (a) Let $L \in(N, M)$. Now we construct a contraction mapping $S_{L}: A(N, M) \rightarrow$ $A(N, M)$ and prove that its fixed point is a bounded positive solution of System (1.14). It follows from (2.3) and (2.4) that there exist $\theta_{L} \in(0,1)$ and $T_{L} \geq \max \left\{\tau_{1}, \tau_{2}\right\}+n_{1}+|\alpha|$ satisfying

$$
\begin{gathered}
\theta_{L}=\sum_{m=T_{L}}^{\infty}(1+m) \max \left\{t_{i m}: i \in \Lambda_{2}\right\} \\
\sum_{m=T_{L}}^{\infty}(1+m) \max \left\{r_{i m}+\left|q_{i m}\right|: i \in \Lambda_{2}\right\}<\min \{M-L, L-N\} .
\end{gathered}
$$

Define three mappings $S_{L}: A(N, M) \rightarrow l_{\alpha}^{\infty} \times l_{\alpha}^{\infty}, S_{1 L}, S_{2 L}: A(N, M) \rightarrow l_{\alpha}^{\infty}$ by

$$
\begin{gathered}
S_{1 L}\left(x_{n}, y_{n}\right)= \begin{cases}L-\sum_{l=0}^{\infty} \sum_{m=n+(l+1) \tau_{1}}^{\infty}\left[f_{1}\left(m, x_{a_{1 m}}, \ldots, x_{a_{h m}}, y_{b_{1 m}}, \ldots, y_{b_{k m}}\right)-q_{1 m}\right], & n \geq T_{L}, \\
S_{1 L}\left(x_{T_{L}}, y_{T_{L}}\right), & \alpha \leq n<T_{L},\end{cases} \\
S_{2 L}\left(x_{n}, y_{n}\right)= \begin{cases}L-\sum_{l=0}^{\infty} \sum_{m=n+(l+1) \tau_{2}}^{\infty}\left[f_{2}\left(m, x_{c_{1 m}}, \ldots, x_{c_{h m}}, y_{d_{1 m}}, \ldots, y_{d_{k m}}\right)-q_{2 m}\right], & n \geq T_{L}, \\
S_{2 L}\left(x_{T_{L}}, y_{T_{L}}\right), & \alpha \leq n<T_{L},\end{cases} \\
S_{L}\left(x_{n}, y_{n}\right)=\left(S_{1 L}\left(x_{n}, y_{n}\right), S_{2 L}\left(x_{n}, y_{n}\right)\right), \quad n \in \mathbb{Z}_{\alpha \prime}
\end{gathered}
$$

for all $(x, y)=\left(\left\{x_{n}\right\}_{n \in \mathbb{Z}_{\alpha}},\left\{y_{n}\right\}_{n \in \mathbb{Z}_{\alpha}}\right) \in A(N, M)$. It follows from (2.1), (2.3), (2.12), (2.13), and Lemma 1.2 that $S_{1 L}$ and $S_{2 L}$ are well defined. 
In view of (2.1), (2.2), and (2.10)-(2.14), we know that for any $(x, y)=\left(\left\{x_{n}\right\}_{n \in \mathbb{Z}_{\alpha^{\prime}}}\right.$ $\left.\left\{y_{n}\right\}_{n \in \mathbb{Z}_{\alpha}}\right) \in A(N, M)$ and $(u, v)=\left(\left\{u_{n}\right\}_{n \in \mathbb{Z}_{\alpha}},\left\{v_{n}\right\}_{n \in \mathbb{Z}_{\alpha}}\right) \in A(N, M)$,

$$
\begin{aligned}
& \left\|S_{L}(x, y)-S_{L}(u, v)\right\|_{1} \\
& =\max \left\{\left\|S_{i L}(x, y)-S_{i L}(u, v)\right\|: i \in \Lambda_{2}\right\} \\
& =\max \left\{\sup _{n \geq T_{L}}\left|S_{i L}\left(x_{n}, y_{n}\right)-S_{i L}\left(u_{n}, v_{n}\right)\right|: i \in \Lambda_{2}\right\} \\
& \leq \max \left\{\operatorname { s u p } _ { n \geq T _ { L } } \left\{\sum_{l=0}^{\infty} \sum_{m=n+(l+1) \tau_{1}}^{\infty} \mid f_{1}\left(m, x_{a_{1 m}}, \ldots, x_{a_{h m}}, y_{b_{1 m}}, \ldots, y_{b_{k m}}\right)\right.\right. \\
& \left.-f_{1}\left(m, u_{a_{1 m}}, \ldots, u_{a_{h m}}, v_{b_{1 m}}, \ldots, v_{b_{k m}}\right) \mid\right\}, \\
& \sup _{n \geq T_{L}}\left\{\sum_{l=0}^{\infty} \sum_{m=n+(l+1) \tau_{2}}^{\infty} \mid f_{2}\left(m, x_{c_{1 m}}, \ldots, x_{c_{h m}}, y_{d b_{1 m}}, \ldots, y_{d_{k m}}\right)\right. \\
& \left.\left.-f_{2}\left(m, u_{c_{1 m}}, \ldots, u_{c_{h m}}, v_{d_{1 m}}, \ldots, v_{d_{k m}}\right) \mid\right\}\right\} \\
& \leq \max \left\{\sum_{l=0}^{\infty} \sum_{m=T_{L}+(l+1) \tau_{1}}^{\infty} t_{1 m} \max \left\{\left|x_{a_{i m}}-u_{a_{i m}}\right|,\left|y_{b_{j m}}-v_{b_{j m}}\right|: i \in \Lambda_{h}, j \in \Lambda_{k}\right\},\right. \\
& \left.\sum_{l=0}^{\infty} \sum_{m=T_{L}+(l+1) \tau_{2}}^{\infty} t_{2 m} \max \left\{\left|x_{c_{i m}}-u_{c_{i m}}\right|,\left|y_{d_{j m}}-v_{d_{j m}}\right|: i \in \Lambda_{h}, j \in \Lambda_{k}\right\}\right\} \\
& \leq \max \left\{\sum_{l=0}^{\infty} \sum_{m=T_{L}+(l+1) \tau_{1}}^{\infty} t_{1 m}, \sum_{l=0}^{\infty} \sum_{m=T_{L}+(l+1) \tau_{2}}^{\infty} t_{2 m}\right\} \max \{\|x-u\|,\|y-v\|\} \\
& \leq \max \left\{\sum_{m=T_{L}}^{\infty}\left(1+\frac{m}{\tau_{1}}\right) t_{1 m}, \sum_{m=T_{L}}^{\infty}\left(1+\frac{m}{\tau_{2}}\right) t_{2 m}\right\}\|(x, y)-(u, v)\|_{1} \\
& \leq\left(\sum_{m=T_{L}}^{\infty}(1+m) \max \left\{t_{1 m}, t_{2 m}\right\}\right)\|(x, y)-(u, v)\|_{1} \\
& =\theta_{L}\|(x, y)-(u, v)\|_{1}, \\
& \max \left\{\left|S_{1 L}\left(x_{n}, y_{n}\right)-L\right|,\left|S_{2 L}\left(x_{n}, y_{n}\right)-L\right|\right\} \\
& \leq \max \left\{\sum_{l=0}^{\infty} \sum_{m=n+(l+1) \tau_{1}}^{\infty}\left|f_{1}\left(m, x_{a_{1 m}}, \ldots, x_{a_{h m}}, y_{b_{1 m}}, \ldots, y_{b_{k m}}\right)-q_{1 m}\right|,\right. \\
& \left.\sum_{l=0}^{\infty} \sum_{m=n+(l+1) \tau_{2}}^{\infty}\left|f_{2}\left(m, x_{c_{1 m}}, \ldots, x_{c_{h m}}, y_{d_{1 m}}, \ldots, y_{d_{k m}}\right)-q_{2 m}\right|\right\}
\end{aligned}
$$


Abstract and Applied Analysis

$$
\begin{aligned}
& \leq \max \left\{\sum_{l=0}^{\infty} \sum_{m=n+(l+1) \tau_{1}}^{\infty}\left(r_{1 m}+\left|q_{1 m}\right|\right), \sum_{l=0}^{\infty} \sum_{m=n+(l+1) \tau_{2}}^{\infty}\left(r_{2 m}+\left|q_{2 m}\right|\right)\right\} \\
& \leq \max \left\{\sum_{m=T_{L}}^{\infty}\left(1+\frac{m}{\tau_{1}}\right)\left(r_{1 m}+\left|q_{1 m}\right|\right), \sum_{m=T_{L}}^{\infty}\left(1+\frac{m}{\tau_{2}}\right)\left(r_{2 m}+\left|q_{2 m}\right|\right)\right\} \\
& \leq \sum_{m=T_{L}}^{\infty}(1+m) \max \left\{r_{i m}+\left|q_{i m}\right|: i \in \Lambda_{2}\right\} \\
& <\min \{M-L, L-N\}, \quad \forall n \geq T_{L \prime}
\end{aligned}
$$

which give that

$$
\begin{gathered}
\left\|S_{L}(x, y)-S_{L}(u, v)\right\|_{1} \leq \theta_{L}\|(x, y)-(u, v)\|_{1}, \quad \forall(x, y),(u, v) \in A(N, M), \\
N \leq S_{1 L}\left(x_{n}, y_{n}\right) \leq M, \quad N \leq S_{2 L}\left(x_{n}, y_{n}\right) \leq M, \quad \forall\left(\left\{x_{n}\right\}_{n \in \mathbb{Z}_{\alpha}},\left\{y_{n}\right\}_{n \in \mathbb{Z}_{\alpha}}\right) \in A(N, M), n \geq T_{L} .
\end{gathered}
$$

Observe that $\theta_{L} \in(0,1)$. It is easy to see that (2.12)-(2.17) give that $S_{L}: A(N, M) \rightarrow$ $A(N, M)$ is a contraction. Consequently, $S_{L}$ possesses a unique fixed point $(x, y)=$ $\left(\left\{x_{n}\right\}_{n \in \mathbb{Z}_{\alpha}},\left\{y_{n}\right\}_{n \in \mathbb{Z}_{\alpha}}\right) \in A(N, M)$, which implies that

$$
\begin{array}{ll}
x_{n}=L-\sum_{l=0}^{\infty} \sum_{m=n+(l+1) \tau_{1}}^{\infty}\left[f_{1}\left(m, x_{a_{1 m}}, \ldots, x_{a_{h m}}, y_{b_{1 m}}, \ldots, y_{b_{k m}}\right)-q_{1 m}\right], & n \geq T_{L}, \\
y_{n}=L-\sum_{l=0}^{\infty} \sum_{m=n+(l+1) \tau_{2}}^{\infty}\left[f_{2}\left(m, x_{c_{1 m}}, \ldots, x_{c_{h m}}, y_{d_{1 m}}, \ldots, y_{d_{k m}}\right)-q_{2 m}\right], \quad n \geq T_{\mathrm{L}},
\end{array}
$$

which yield that

$$
\begin{aligned}
x_{n}-x_{n-\tau_{1}}= & L-\sum_{l=0}^{\infty} \sum_{m=n+(l+1) \tau_{1}}^{\infty}\left[f_{1}\left(m, x_{a_{1 m}}, \ldots, x_{a_{h m}}, y_{b_{1 m}}, \ldots, y_{b_{k m}}\right)-q_{1 m}\right] \\
& -L+\sum_{l=0}^{\infty} \sum_{m=n+l \tau_{1}}^{\infty}\left[f_{1}\left(m, x_{a_{1 m}}, \ldots, x_{a_{h m}}, y_{b_{1 m}}, \ldots, y_{b_{k m}}\right)-q_{1 m}\right] \\
= & \sum_{m=n}^{\infty}\left[f_{1}\left(m, x_{a_{1 m}}, \ldots, x_{a_{h m}}, y_{b_{1 m}}, \ldots, y_{b_{k m}}\right)-q_{1 m}\right], \quad \forall n \geq T_{L}+\tau_{1},
\end{aligned}
$$




$$
\begin{aligned}
y_{n}-y_{n-\tau_{2}}= & L-\sum_{l=0}^{\infty} \sum_{m=n+(l+1) \tau_{2}}^{\infty}\left[f_{2}\left(m, x_{c_{1 m}}, \ldots, x_{c_{h m}}, y_{d_{1 m}}, \ldots, y_{d_{k m}}\right)-q_{2 m}\right] \\
& -L+\sum_{l=0}^{\infty} \sum_{m=n+l \tau_{2}}^{\infty}\left[f_{2}\left(m, x_{c_{1 m}}, \ldots, x_{c_{h m}}, y_{d_{1 m}}, \ldots, y_{d_{k m}}\right)-q_{2 m}\right] \\
= & \sum_{m=n}^{\infty}\left[f_{2}\left(m, x_{c_{1 m}}, \ldots, x_{c_{h m}}, y_{d_{1 m}}, \ldots, y_{d_{k m}}\right)-q_{2 m}\right], \quad \forall n \geq T_{L}+\tau_{2},
\end{aligned}
$$

which give that

$$
\begin{array}{ll}
\Delta\left(x_{n}-x_{n-\tau_{1}}\right)=-f_{1}\left(n, x_{a_{1 n}}, \ldots, x_{a_{h n}}, y_{b_{1 n}}, \ldots, y_{b_{k n}}\right)+q_{1 n}, & \forall n \geq T_{L}+\tau_{1} \\
\Delta\left(y_{n}-y_{n-\tau_{2}}\right)=-f_{2}\left(n, x_{c_{1 n}}, \ldots, x_{c_{h n}}, y_{d_{1 n}}, \ldots, y_{d_{k n}}\right)+q_{2 n}, \quad \forall n \geq T_{L}+\tau_{2}
\end{array}
$$

that is, $(x, y)$ is a bounded positive solution of System $(1.14)$ in $A(N, M)$.

In light of (2.5), (2.6), and (2.11)-(2.16), we arrive at

$$
\begin{aligned}
& \left\|\left(x^{\mu+1}, y^{\mu+1}\right)-(x, y)\right\|_{1} \\
& =\max \left\{\sup _{n \geq T_{L}}\left|x_{n}^{\mu+1}-x_{n}\right|, \sup _{n \geq T_{L}}\left|y_{n}^{\mu+1}-y_{n}\right|\right\} \\
& \leq \max \left\{\operatorname { s u p } _ { n \geq T _ { L } } \left[\left(1-\beta_{\mu}-\gamma_{\mu}\right)\left|x_{n}^{\mu}-x_{n}\right|\right.\right. \\
& +\beta_{\mu}\left|L-\sum_{l=0}^{\infty} \sum_{m=n+(l+1) \tau_{1}}^{\infty}\left[f_{1}\left(m, x_{a_{1 m}}^{\mu}, \ldots, x_{a_{h m}}^{\mu}, y_{b_{1 m}}^{\mu}, \ldots, y_{b_{k m}}^{\mu}\right)-q_{1 m}\right]-x_{n}\right| \\
& \left.+\gamma_{\mu}\left|\delta_{1 n}^{\mu}-x_{n}\right|\right] \\
& \sup _{n \geq T_{L}}\left[\left(1-\beta_{\mu}-\gamma_{\mu}\right)\left|y_{n}^{\mu}-y_{n}\right|\right. \\
& +\beta_{\mu}\left|L-\sum_{l=0}^{\infty} \sum_{m=n+(l+1) \tau_{2}}^{\infty}\left[f_{2}\left(m, x_{c_{1 m}}^{\mu}, \ldots, x_{c_{h m}}^{\mu}, y_{d_{1 m}}^{\mu}, \ldots, y_{d_{k m}}^{\mu}\right)-q_{2 m}\right]-y_{n}\right| \\
& \left.\left.+\gamma_{\mu}\left|\delta_{2 n}^{\mu}-y_{n}\right|\right]\right\}
\end{aligned}
$$




$$
\begin{gathered}
\leq \max \left\{\sup _{n \geq T_{L}}\left[\left(1-\beta_{\mu}-\gamma_{\mu}\right)\left|x_{n}^{\mu}-x_{n}\right|+\beta_{\mu}\left|S_{1 L}\left(x_{n}^{\mu}, y_{n}^{\mu}\right)-S_{1 L}\left(x_{n}, y_{n}\right)\right|+2 M \gamma_{\mu}\right],\right. \\
\left.\sup _{n \geq T_{L}}\left[\left(1-\beta_{\mu}-\gamma_{\mu}\right)\left|y_{n}^{\mu}-y_{n}\right|+\beta_{\mu}\left|S_{2 L}\left(x_{n}^{\mu}, y_{n}^{\mu}\right)-S_{2 L}\left(x_{n}, y_{n}\right)\right|+2 M \gamma_{\mu}\right]\right\} \\
\leq \max \left\{\left(1-\beta_{\mu}-\gamma_{\mu}\right)\left\|x^{\mu}-x\right\|+\theta_{L} \beta_{\mu}\left\|\left(x^{\mu}, y^{\mu}\right)-(x, y)\right\|_{1}+2 M \gamma_{\mu},\right. \\
\left.\quad\left(1-\beta_{\mu}-\gamma_{\mu}\right)\left\|y^{\mu}-y\right\|+\theta_{L} \beta_{\mu}\left\|\left(x^{\mu}, y^{\mu}\right)-(x, y)\right\|_{1}+2 M \gamma_{\mu}\right\} \\
\leq\left(1-\beta_{\mu}\left(1-\theta_{L}\right)\right)\left\|\left(x^{\mu}, y^{\mu}\right)-(x, y)\right\|_{1}+2 M \gamma_{\mu}, \quad \forall \mu \geq 0,
\end{gathered}
$$

that is, (2.7) holds. It follows from (2.7)-(2.9) and Lemma 1.1 that $\lim _{\mu \rightarrow \infty}\left\|\left(x^{\mu}, y^{\mu}\right)-(x, y)\right\|_{1}=$ 0 .

(b) Let $L_{1}, L_{2} \in(N, M)$, and $L_{1} \neq L_{2}$. As in the proof of (a), we similarly infer that for each $i \in \Lambda_{2}$, there exist a constant $\theta_{L_{i}} \in(0,1)$, a positive integer $T_{L_{i}} \geq \max \left\{\tau_{1}, \tau_{2}\right\}+n_{1}+|\alpha|$, and mappings $S_{L_{i}}, S_{1 L_{i}}, S_{2 L_{i}}$ satisfying (2.10)-(2.14), where $L, \theta_{L}$ and $T_{L}$ are replaced by $L_{i}, \theta_{L_{i}}$ and $T_{L_{i}}$, respectively, and the contraction mappings $S_{L_{1}}$ and $S_{L_{2}}$ have the unique fixed points $(u, v)=\left(\left\{u_{n}\right\}_{n \in \mathbb{Z}_{\alpha}}\left\{v_{n}\right\}_{n \in \mathbb{Z}_{\alpha}}\right),(w, z)=\left(\left\{w_{n}\right\}_{n \in \mathbb{Z}_{\alpha^{\prime}}}\left\{z_{n}\right\}_{n \in \mathbb{Z}_{\alpha}}\right) \in A(N, M)$, respectively, $(u, v)$ and $(w, z)$ are bounded positive solutions of System $(1.14)$ in $A(N, M)$. In order to show that System (1.14) possesses uncountably many bounded positive solutions in $A(N, M)$, we prove only that $(u, v) \neq(w, z)$. By means of (2.10) and (2.12)-(2.16), we conclude that

$$
\begin{aligned}
& \|(u, v)-(w, z)\|_{1} \\
& =\max \{\|u-w\|,\|v-z\|\} \\
& \geq \max \left\{\sup _{n \geq \max \left\{T_{L_{1}}, T_{L_{2}}\right\}}\left|u_{n}-w_{n}\right|, \sup _{n \geq \max \left\{T_{L_{1}}, T_{L_{2}}\right\}}\left|v_{n}-z_{n}\right|\right\} \\
& \geq \max \left\{\left|L_{1}-L_{2}\right|-\sum_{l=0}^{\infty} \sum_{m=\max \left\{T_{L_{1}}, T_{L_{2}}\right\}+(l+1) \tau_{1}}^{\infty} \mid f_{1}\left(m, u_{a_{1 m}}, \ldots, u_{a_{h m}}, v_{b_{1 m}}, \ldots, v_{b_{k m}}\right)\right. \\
& \\
& \left|L_{1}-L_{2}\right|-\sum_{l=0}^{\infty} \sum_{m=\max \left\{T_{L_{1}}, T_{L_{2}}\right\}+(l+1) \tau_{2}}^{\infty} \mid f_{2}\left(m, u_{c_{1 m}}, \ldots, u_{c_{h m}}, v_{d_{1 m}}, \ldots, v_{d_{k m}}\right) \\
& \geq \max \left\{\left|L_{1}-L_{2}\right|-\sum_{l=0}^{\infty} \sum_{m=\max \left\{T_{\left.L_{1}, T_{L_{2}}\right\}+(l+1) \tau_{1}}^{\infty} t_{1 m} \max \left\{\left|u_{a_{i m}}-w_{a_{i m}}\right|,\left|v_{b_{j m}}-z_{b_{j m}}\right|: i \in \Lambda_{h}, j \in \Lambda_{K}\right\},\right.}^{\infty},\right.
\end{aligned}
$$




$$
\begin{gathered}
\left|L_{1}-L_{2}\right|-\sum_{l=0}^{\infty} \sum_{m=\max \left\{T_{L_{1}}, T_{L_{2}}\right\}+(l+1) \tau_{2}}^{\infty} t_{2 m} \\
\left.\times \max \left\{\left|u_{c_{i m}}-w_{c_{i m}}\right|,\left|v_{d_{j m}}-z_{d_{j m}}\right|: i \in \Lambda_{h}, j \in \Lambda_{K}\right\}\right\} \\
\geq \max \left\{\left|L_{1}-L_{2}\right|-\sum_{l=0}^{\infty} \sum_{m=\max \left\{T_{L_{1}}, T_{L_{2}}\right\}+(l+1) \tau_{1}}^{\infty} t_{1 m} \max \{\|u-w\|,\|v-z\|\},\right. \\
\left.\left|L_{1}-L_{2}\right|-\sum_{l=0}^{\infty} \sum_{m=\max \left\{T_{L_{1}}, T_{L_{2}}\right\}+(l+1) \tau_{2}}^{\infty} t_{2 m} \max \{\|u-w\|,\|v-z\|\}\right\} \\
\geq\left|L_{1}-L_{2}\right|-\left(\sum_{m=\max \left\{T_{L_{1}}, T_{L_{2}}\right\}}^{\infty}(1+m) \max \left\{t_{i m}: i \in \Lambda_{2}\right\}\right)\|(u, v)-(w, z)\|_{1} \\
\geq\left|L_{1}-L_{2}\right|-\max \left\{\theta_{L_{1}}, \theta_{L_{2}}\right\}\|(u, v)-(w, z)\|_{1},
\end{gathered}
$$

which implies that

$$
\|(u, v)-(w, z)\|_{1} \geq \frac{\left|L_{1}-L_{2}\right|}{1+\max \left\{\theta_{L_{1}}, \theta_{L_{2}}\right\}}>0,
$$

that is, $(u, v) \neq(w, z)$. This completes the proof.

Theorem 2.2. Assume that there exist constants $M, N \in \mathbb{R}^{+} \backslash\{0\}, n_{1} \in \mathbb{N}_{n_{0}}$, and four nonnegative sequences $\left\{r_{1 n}\right\}_{n \in \mathbb{N}_{n_{0}}},\left\{r_{2 n}\right\}_{n \in \mathbb{N}_{n_{0}}},\left\{t_{1 n}\right\}_{n \in \mathbb{N}_{n_{0}}}$, and $\left\{t_{2 n}\right\}_{n \in \mathbb{N}_{n_{0}}}$ satisfying (2.1), (2.2),

$$
\begin{aligned}
& \sum_{n=n_{0}}^{\infty} \max \left\{r_{i n}, t_{i n},\left|q_{i n}\right|: i \in \Lambda_{2}\right\}<+\infty, \\
& N<M, \quad p_{i n}=1, \quad \forall n \geq n_{1}, i \in \Lambda_{2} .
\end{aligned}
$$


Then,

(a) for each $L \in(N, M)$, there exist $\theta_{L} \in(0,1)$ and $T_{L} \geq \max \left\{\tau_{1}, \tau_{2}\right\}+n_{1}+|\alpha|$ such that for any $\left(x^{0}, y^{0}\right)=\left(\left\{x_{n}^{0}\right\}_{n \in \mathbb{Z}_{\alpha}},\left\{y_{n}^{0}\right\}_{n \in \mathbb{Z}_{\alpha}}\right) \in A(N, M)$, the Mann iterative sequence with errors $\left\{\left(x^{\mu}, y^{\mu}\right)\right\}_{\mu \in \mathbb{N}_{0}}=\left\{\left(\left\{x_{n}^{\mu}\right\}_{n \in \mathbb{Z}_{\alpha}},\left\{y_{n}^{\mu}\right\}_{n \in \mathbb{Z}_{\alpha}}\right)\right\}_{\mu \in \mathbb{N}_{0}}$ generated by the schemes

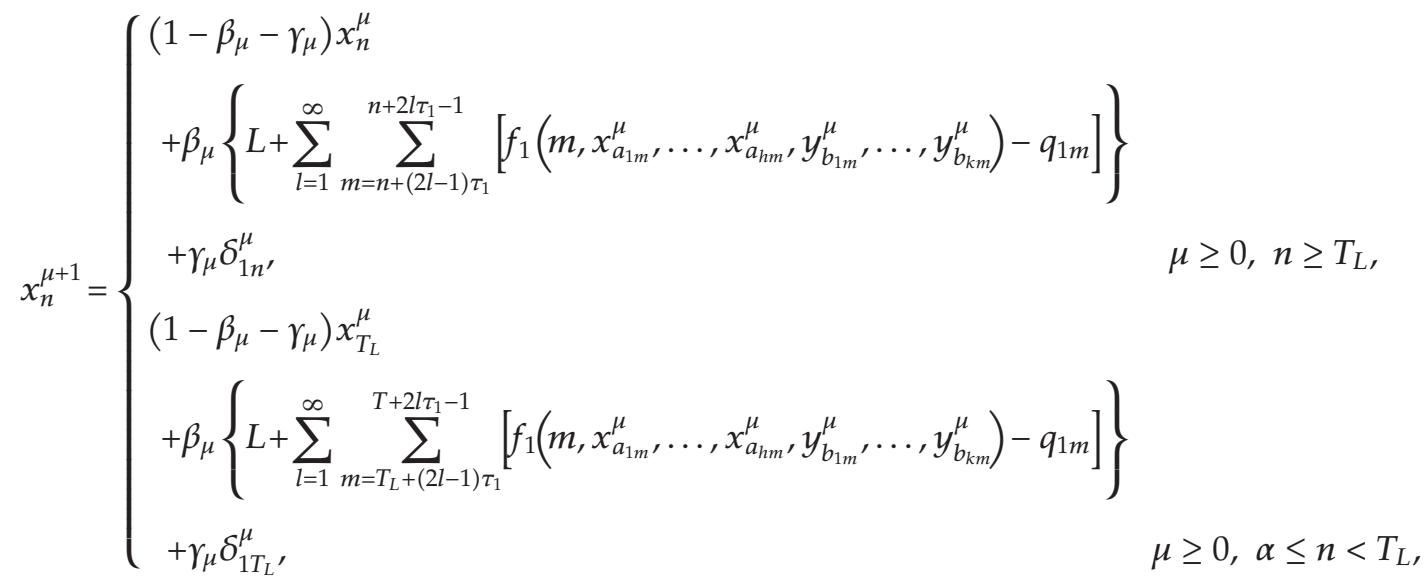

$$
y_{n}^{\mu+1}= \begin{cases}\left(1-\beta_{\mu}-\gamma_{\mu}\right) y_{n}^{\mu} & \\ +\beta_{\mu}\left\{L+\sum_{l=1}^{\infty} \sum_{m=n+(2 l-1) \tau_{2}}^{n+2 l \tau_{2}-1}\left[f_{2}\left(m, x_{c_{1 m}}^{\mu}, \ldots, x_{c_{h m}}^{\mu}, y_{d_{1 m}}^{\mu}, \ldots, y_{d_{k m}}^{\mu}\right)-q_{2 m}\right]\right\} & \\ +\gamma_{\mu} \delta_{2 n^{\prime}}^{\mu} & \mu \geq 0, n \geq T_{L}, \\ \left(1-\beta_{\mu}-\gamma_{\mu}\right) y_{T_{L}}^{\mu} & \\ +\beta_{\mu}\left\{L+\sum_{l=1}^{\infty} \sum_{m=T_{L}+(2 l-1) \tau_{2}}^{T_{L}+2 l \tau_{2}-1}\left[f_{2}\left(m, x_{c_{1 m}}^{\mu}, \ldots, x_{c_{h m}}^{\mu}, y_{d_{1 m^{\prime}}}^{\mu}, \ldots, y_{d_{k m}}^{\mu}\right)-q_{2 m}\right]\right\} & \\ +\gamma_{\mu} \delta_{2 T_{L}}^{\mu}, & \mu \geq 0, \alpha \leq n<T_{L}\end{cases}
$$

converges to a bounded positive solution $(x, y) \in A(N, M)$ of System (1.14) and has the error estimate (2.7), where $\left\{\beta_{\mu}\right\}_{\mu \in \mathbb{N}_{0}},\left\{\gamma_{\mu}\right\}_{\mu \in \mathbb{N}_{0}} \subset[0,1]$, and $\left\{\left(\delta_{1}^{\mu}, \delta_{2}^{\mu}\right)\right\}_{\mu \in \mathbb{N}_{0}}=\left\{\left(\left\{\delta_{1 n}^{\mu}\right\}_{n \in \mathbb{Z}_{\alpha^{\prime}}}\right.\right.$ $\left.\left.\left\{\delta_{2 n}^{\mu}\right\}_{n \in \mathbb{Z}_{\alpha}}\right)\right\}_{\mu \in \mathbb{N}_{0}} \subset A(N, M)$ are arbitrary sequences with (2.8) and (2.9),

(b) System (1.14) has uncountably many bounded positive solutions in $A(N, M)$.

Proof. (a) Let $L \in(N, M)$. Now we construct a contraction mapping $S_{L}: A(N, M) \rightarrow$ $A(N, M)$ and prove that its fixed point is a bounded positive solution of System (1.14). Note 
that (2.24) and (2.25) guarantee that there exist $\theta_{L} \in(0,1)$ and $T_{L} \geq \max \left\{\tau_{1}, \tau_{2}\right\}+n_{1}+|\alpha|$ satisfying

$$
\begin{gathered}
\theta_{L}=\sum_{m=T_{L}}^{\infty} \max \left\{t_{i m}: i \in \Lambda_{2}\right\}, \\
\sum_{m=T_{L}}^{\infty} \max \left\{r_{i m}+\left|q_{i m}\right|: i \in \Lambda_{2}\right\}<\min \{M-L, L-N\} .
\end{gathered}
$$

Define three mappings $S_{L}: A(N, M) \rightarrow l_{\alpha}^{\infty} \times l_{\alpha}^{\infty}, S_{1 L}, S_{2 L}: A(N, M) \rightarrow l_{\alpha}^{\infty}$ by (2.14),

$$
\begin{aligned}
& S_{1 L}\left(x_{n}, y_{n}\right)= \begin{cases}L+\sum_{l=1}^{\infty} \sum_{m=n+(2 l-1) \tau_{1}}^{n+2 l \tau_{1}-1}\left[f_{1}\left(m, x_{a_{1 m}}, \ldots, x_{a_{h m}}, y_{b_{1 m}}, \ldots, y_{b_{k m}}\right)-q_{1 m}\right], & n \geq T_{L}, \\
S_{1 L}\left(x_{T_{L}}, y_{T_{L}}\right), & \alpha \leq n<T_{L},\end{cases} \\
& S_{2 L}\left(x_{n}, y_{n}\right)= \begin{cases}L+\sum_{l=1}^{\infty} \sum_{m=n+(2 l-1) \tau_{2}}^{n+2 l \tau_{2}-1}\left[f_{2}\left(m, x_{c_{1 m}}, \ldots, x_{c_{h m}}, y_{d_{1 m}}, \ldots, y_{d_{k m}}\right)-q_{2 m}\right], & n \geq T_{L}, \\
S_{2 L}\left(x_{T_{L}}, y_{T_{L}}\right), & \alpha \leq n<T_{L},\end{cases}
\end{aligned}
$$

for all $(x, y)=\left(\left\{x_{n}\right\}_{n \in \mathbb{Z}_{\alpha}},\left\{y_{n}\right\}_{n \in \mathbb{Z}_{\alpha}}\right) \in A(N, M)$.

Using (2.1), (2.2), (2.14), and (2.28)-(2.31), we deduce that for any $(x, y)=$ $\left(\left\{x_{n}\right\}_{n \in \mathbb{Z}_{\alpha}}\left\{y_{n}\right\}_{n \in \mathbb{Z}_{\alpha}}\right)$ and $(u, v)=\left(\left\{u_{n}\right\}_{n \in \mathbb{Z}_{\alpha}}\left\{v_{n}\right\}_{n \in \mathbb{Z}_{\alpha}}\right) \in A(N, M)$,

$$
\begin{aligned}
& \left\|S_{L}(x, y)-S_{L}(u, v)\right\|_{1} \\
& =\max \left\{\left\|S_{i L}(x, y)-S_{i L}(u, v)\right\|: i \in \Lambda_{2}\right\} \\
& =\max \left\{\sup _{n \geq T_{L}}\left|S_{i L}\left(x_{n}, y_{n}\right)-S_{i L}\left(u_{n}, v_{n}\right)\right|: i \in \Lambda_{2}\right\} \\
& \leq \max \left\{\operatorname { s u p } _ { n \geq T _ { L } } \left\{\sum_{l=1}^{\infty} \sum_{m=n+(2 l-1) \tau_{1}}^{m=n+2 l \tau_{1}-1} \mid f_{1}\left(m, x_{a_{1 m}}, \ldots, x_{a_{h m}}, y_{b_{1 m}}, \ldots, y_{b_{k m}}\right)\right.\right. \\
& \sup _{n \geq T_{L}}\left\{\sum_{l=1}^{\infty} \sum_{m=n+(2 l-1) \tau_{2}}^{m=n+2 l \tau_{2}-1} \mid f_{2}\left(m, x_{c_{1 m}}, \ldots, x_{c_{h m}}, y_{d_{1 m}}, \ldots, y_{d_{k m}}\right)\right. \\
& \left.-f_{2}\left(m, u_{a_{1 m}}, \ldots, u_{a_{h m}}, v_{b_{1 m}}, \ldots, v_{b_{k m}}\right) \mid\right\}, \\
& \left.\left.\left.u_{c_{1 m}}, \ldots, u_{c_{h m}}, v_{d_{1 m}}, \ldots, v_{d_{k m}}\right) \mid\right\}\right\}
\end{aligned}
$$


Abstract and Applied Analysis

$$
\begin{aligned}
& \leq \max \left\{\sum_{m=T_{L}+\tau_{1}}^{\infty} t_{1 m} \max \left\{\left|x_{a_{i m}}-u_{a_{i m}}\right|,\left|y_{b_{j m}}-v_{b_{j m}}\right|: i \in \Lambda_{h}, j \in \Lambda_{k}\right\},\right. \\
& \left.\sum_{m=T_{L}+\tau_{2}}^{\infty} t_{2 m} \max \left\{\left|x_{c_{i m}}-u_{c_{i m}}\right|,\left|y_{d_{j m}}-v_{d_{j m}}\right|: i \in \Lambda_{h}, j \in \Lambda_{k}\right\}\right\} \\
& \leq \max \left\{\sum_{m=T_{L}+\tau_{1}}^{\infty} t_{1 m}, \sum_{m=T_{L}+\tau_{2}}^{\infty} t_{2 m}\right\} \max \{\|x-u\|,\|y-v\|\} \\
& \leq\left(\sum_{m=T_{L}}^{\infty} \max \left\{t_{i m}: i \in \Lambda_{2}\right\}\right)\|(x, y)-(u, v)\|_{1} \\
& =\theta_{L}\|(x, y)-(u, v)\|_{1} \text {, } \\
& \max \left\{\left|S_{1 L}\left(x_{n}, y_{n}\right)-L\right|,\left|S_{2 L}\left(x_{n}, y_{n}\right)-L\right|\right\} \\
& \leq \max \left\{\sum_{l=1}^{\infty} \sum_{m=n+(2 l-1) \tau_{1}}^{n+2 l \tau_{1}-1}\left|f_{1}\left(m, x_{a_{1 m}}, \ldots, x_{a_{h m}}, y_{b_{1 m}}, \ldots, y_{b_{k m}}\right)-q_{1 m}\right|,\right. \\
& \left.\sum_{l=1}^{\infty} \sum_{m=n+(2 l-1) \tau_{2}}^{n+2 l \tau_{2}-1}\left|f_{2}\left(m, x_{c_{1 m}}, \ldots, x_{c_{h m}}, y_{d_{1 m}}, \ldots, y_{d_{k m}}\right)-q_{2 m}\right|\right\} \\
& \leq \max \left\{\sum_{l=1}^{\infty} \sum_{m=n+(2 l-1) \tau_{1}}^{n+2 l \tau_{1}-1}\left(r_{1 m}+\left|q_{1 m}\right|\right), \sum_{l=1}^{\infty} \sum_{m=n+(2 l-1) \tau_{2}}^{n+2 l \tau_{2}-1}\left(r_{2 m}+\left|q_{2 m}\right|\right)\right\} \\
& \leq \max \left\{\sum_{m=T_{L}+\tau_{1}}^{\infty}\left(r_{1 m}+\left|q_{1 m}\right|\right), \sum_{m=T_{L}+\tau_{2}}^{\infty}\left(r_{2 m}+\left|q_{2 m}\right|\right)\right\} \\
& \leq \sum_{m=T_{L}}^{\infty} \max \left\{r_{i m}+\left|q_{i m}\right|: i \in \Lambda_{2}\right\} \\
& <\min \{M-L, L-N\}, \quad \forall n \geq T_{L},
\end{aligned}
$$

which imply (2.16) and (2.17) and which ensure that $S_{L}$ is a self-mapping from $A(N, M)$ into itself and is a contraction by $\theta_{L} \in(0,1)$. The Banach fixed point theorem means that $S_{L}$ has a unique fixed point $(x, y)=\left(\left\{x_{n}\right\}_{n \in \mathbb{Z}_{\alpha}},\left\{y_{n}\right\}_{n \in \mathbb{Z}_{\alpha}}\right) \in A(N, M)$, that is,

$$
\begin{array}{ll}
x_{n}=L+\sum_{l=1}^{\infty} \sum_{m=n+(2 l-1) \tau_{1}}^{n+2 l \tau_{1}-1}\left[f_{1}\left(m, x_{a_{1 m}}, \ldots, x_{a_{h m}}, y_{b_{1 m}}, \ldots, y_{b_{k m}}\right)-q_{1 m}\right], \quad n \geq T_{L}, \\
y_{n}=L+\sum_{l=1}^{\infty} \sum_{m=n+(2 l-1) \tau_{2}}^{n+2 l \tau_{2}-1}\left[f_{2}\left(m, x_{c_{1 m}}, \ldots, x_{c_{h m}}, y_{d_{1 m}}, \ldots, y_{d_{k m}}\right)-q_{2 m}\right], \quad n \geq T_{L},
\end{array}
$$


which reveal that

$$
\begin{aligned}
x_{n}+x_{n-\tau_{1}}= & L+\sum_{l=1}^{\infty} \sum_{m=n+(2 l-1) \tau_{1}}^{n+2 l \tau_{1}-1}\left[f_{1}\left(m, x_{a_{1 m}}, \ldots, x_{a_{h m}}, y_{b_{1 m}}, \ldots, y_{b_{k m}}\right)-q_{1 m}\right] \\
& +L+\sum_{l=1}^{\infty} \sum_{m=n+2(l-1) \tau_{1}}^{n+(2 l-1) \tau_{1}-1}\left[f_{1}\left(m, x_{a_{1 m}}, \ldots, x_{a_{h m}}, y_{b_{1 m}}, \ldots, y_{b_{k m}}\right)-q_{1 m}\right] \\
= & 2 L+\sum_{m=n}^{\infty}\left[f_{1}\left(m, x_{\mathrm{a}_{1 m}}, \ldots, x_{a_{h m}}, y_{b_{1 m}}, \ldots, y_{b_{k m}}\right)-q_{1 m}\right], \quad \forall n \geq T_{L}+\tau_{1}, \\
y_{n}+y_{n-\tau_{2}}= & L+\sum_{l=1}^{\infty} \sum_{m=n+(2 l-1) \tau_{2}}^{n+2 l \tau_{2}-1}\left[f_{2}\left(m, x_{c_{1 m}}, \ldots, x_{c_{h m}}, y_{d_{1 m}}, \ldots, y_{d_{k m}}\right)-q_{2 m}\right] \\
& +L+\sum_{l=1}^{\infty} \sum_{m=n+2(l-1) \tau_{2}}^{n+(2 l-1) \tau_{2}-1}\left[f_{2}\left(m, x_{c_{1 m}}, \ldots, x_{c_{h m}}, y_{d_{1 m}}, \ldots, y_{d_{k m}}\right)-q_{2 m}\right] \\
= & 2 L+\sum_{m=n}^{\infty}\left[f_{2}\left(m, x_{c_{1 m}}, \ldots, x_{c_{h m}}, y_{d_{1 m}}, \ldots, y_{d_{k m}}\right)-q_{2 m}\right], \quad \forall n \geq T_{L}+\tau_{2},
\end{aligned}
$$

which yield that

$$
\begin{array}{ll}
\Delta\left(x_{n}-x_{n-\tau_{1}}\right)=-f_{1}\left(n, x_{a_{1 n}}, \ldots, x_{a_{h n}}, y_{b_{1 n}}, \ldots, y_{b_{k n}}\right)+q_{1 n}, & \forall n \geq T_{L}+\tau_{1}, \\
\Delta\left(y_{n}-y_{n-\tau_{2}}\right)=-f_{2}\left(n, x_{c_{1 n}}, \ldots, x_{c_{h n}}, y_{d_{1 n}}, \ldots, y_{d_{k n}}\right)+q_{2 n}, & \forall n \geq T_{L}+\tau_{2},
\end{array}
$$

that is, $(x, y)$ is a bounded positive solution of System $(1.14)$ in $A(N, M)$.

It follows from $(2.14),(2.16),(2.26)-(2.28),(2.30)$, and (2.31) that

$$
\begin{gathered}
\left\|\left(x^{\mu+1}, y^{\mu+1}\right)-(x, y)\right\|_{1} \\
=\max \left\{\sup _{n \geq T_{L}}\left|x_{n}^{\mu+1}-x_{n}\right|, \sup _{n \geq T_{L}}\left|y_{n}^{\mu+1}-y_{n}\right|\right\} \\
\leq \max \left\{\operatorname { s u p } _ { n \geq T _ { L } } \left[\left(1-\beta_{\mu}-\gamma_{\mu}\right)\left|x_{n}^{\mu}-x_{n}\right|\right.\right. \\
+\beta_{\mu}\left|L+\sum_{l=1}^{\infty} \sum_{m=n+(2 l-1) \tau_{1}}^{n+2 l \tau_{1}-1}\left[f_{1}\left(m, x_{a_{1 m}}^{\mu}, \ldots, x_{a_{h m}}^{\mu}, y_{b_{1 m}}^{\mu}, \ldots, y_{b_{k m}}^{\mu}\right)-q_{1 m}\right]-x_{n}\right| \\
\left.+\gamma_{\mu}\left|\delta_{1 n}^{\mu}-x_{n}\right|\right],
\end{gathered}
$$




$$
\begin{aligned}
& \sup _{n \geq T_{L}}\left[\left(1-\beta_{\mu}-\gamma_{\mu}\right)\left|y_{n}^{\mu}-y_{n}\right|\right. \\
& \quad+\beta_{\mu}\left|L+\sum_{l=1}^{\infty} \sum_{m=n+(2 l-1) \tau_{2}}^{n+2 l \tau_{2}-1}\left[f_{2}\left(m, x_{c_{1 m}}^{\mu}, \ldots, x_{c_{h m}}^{\mu}, y_{d_{1 m}}^{\mu}, \ldots, y_{d_{k m}}^{\mu}\right)-q_{2 m}\right]-y_{n}\right| \\
& \left.\left.\quad+\gamma_{\mu}\left|\delta_{2 n}^{\mu}-y_{n}\right|\right]\right\} \\
& \leq \max \left\{\sup _{n \geq T_{L}}\left[\left(1-\beta_{\mu}-\gamma_{\mu}\right)\left|x_{n}^{\mu}-x_{n}\right|+\beta_{\mu}\left|S_{1 L}\left(x_{n}^{\mu}, y_{n}^{\mu}\right)-S_{1 L}\left(x_{n}, y_{n}\right)\right|+2 M \gamma_{\mu}\right],\right. \\
& \left.\sup _{n \geq T_{L}}\left[\left(1-\beta_{\mu}-\gamma_{\mu}\right)\left|y_{n}^{\mu}-y_{n}\right|+\beta_{\mu}\left|S_{2 L}\left(x_{n}^{\mu}, y_{n}^{\mu}\right)-S_{2 L}\left(x_{n}, y_{n}\right)\right|+2 M \gamma_{\mu}\right]\right\} \\
& \leq\left(1-\beta_{\mu}\left(1-\theta_{L}\right)\right)\left\|\left(x^{\mu}, y^{\mu}\right)-(x, y)\right\|_{1}+2 M \gamma_{\mu}, \quad \forall \mu \geq 0,
\end{aligned}
$$

which implies (2.7). Thus Lemma 1.1 and (2.7)-(2.9) mean that $\lim _{\mu \rightarrow \infty}\left\|\left(x^{\mu}, y^{\mu}\right)-(x, y)\right\|_{1}=0$.

(b) Let $L_{1}, L_{2} \in(N, M)$ and $L_{1} \neq L_{2}$. As in the proof of (a), we conclude that for each $i \in \Lambda_{2}$, there exist a constant $\theta_{L_{i}} \in(0,1)$, a positive integer $T_{L_{i}} \geq \max \left\{\tau_{1}, \tau_{2}\right\}+n_{1}+|\alpha|$, and mappings $S_{L_{i}}, S_{1 L_{i}}, S_{2 L_{i}}$ satisfying (2.14) and (2.28)-(2.31), where $L, \theta_{L}$, and $T_{L}$ are replaced by $L_{i}, \theta_{L_{i}}$, and $T_{L_{i}}$, respectively, and the contraction mappings $S_{L_{1}}$ and $S_{L_{2}}$ have the unique fixed points $(u, v)=\left(\left\{u_{n}\right\}_{n \in \mathbb{Z}_{\alpha}},\left\{v_{n}\right\}_{n \in \mathbb{Z}_{\alpha}}\right),(w, z)=\left(\left\{w_{n}\right\}_{n \in \mathbb{Z}_{\alpha}},\left\{z_{n}\right\}_{n \in \mathbb{Z}_{\alpha}}\right) \in A(N, M)$, respectively, $(u, v)$ and $(w, z)$ are bounded positive solutions of the system $(1.14)$ in $A(N, M)$. In order to show that System (1.14) possesses uncountably many bounded positive solutions in $A(N, M)$, we prove only that $(u, v) \neq(w, z)$. By virtue of (2.14) and (2.28)-(2.31), we infer that

$$
\begin{aligned}
& \|(u, v)-(w, z)\|_{1} \\
& =\max \{\|u-w\|,\|v-z\|\} \\
& \geq \max \left\{\sup _{n \geq \max \left\{T_{L_{1}}, T_{L_{2}}\right\}}\left|u_{n}-w_{n}\right|, \sup _{n \geq \max \left\{T_{L_{1}}, T_{L_{2}}\right\}}\left|v_{n}-z_{n}\right|\right\} \\
& \geq \max \left\{\left|L_{1}-L_{2}\right|-\sum_{l=1}^{\infty} \sum_{m=\max \left\{T_{L_{1}}, T_{L_{2}}\right\}+(2 l-1) \tau_{1}}^{\max \left\{T_{L_{1}}, T_{L_{2}}\right\}+2 l \tau_{1}-1} \mid f_{1}\left(m, u_{a_{1 m}}, \ldots, u_{a_{h m}}, v_{b_{1 m}}, \ldots, v_{b_{k m}}\right)\right. \\
& -f_{1}\left(m, w_{a_{1 m}}, \ldots, w_{a_{h m}}, z_{b_{1 m}}, \ldots, z_{b_{k m}}\right) \mid,
\end{aligned}
$$




$$
\begin{aligned}
& \left|L_{1}-L_{2}\right|-\sum_{l=1}^{\infty} \sum_{m=\max \left\{T_{L_{1}}, T_{L_{2}}\right\}+(2 l-1) \tau_{2}}^{\max \left\{T_{L_{1}}, T_{L_{2}}\right\}+2 l \tau_{2}-1} \mid f_{2}\left(m, u_{c_{1 m}}, \ldots, u_{c_{h m}}, v_{d_{1 m}}, \ldots, v_{d_{k m}}\right) \\
& \left.-f_{2}\left(m, w_{c_{1 m}}, \ldots, w_{c_{h m}}, z_{d_{1 m}}, \ldots, z_{d_{k m}}\right) \mid\right\}
\end{aligned}
$$

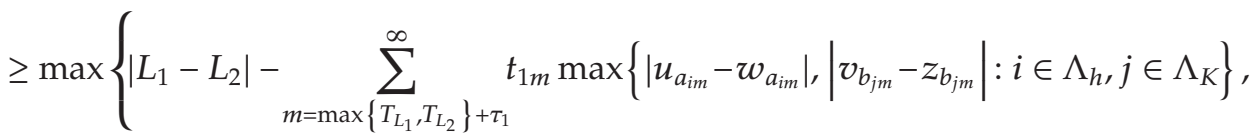

$$
\begin{aligned}
& \left.\left|L_{1}-L_{2}\right|-\sum_{m=\max \left\{T_{L_{1}}, T_{L_{2}}\right\}+\tau_{2}}^{\infty} t_{2 m} \max \left\{\left|u_{c_{i m}}-w_{c_{i m}}\right|,\left|v_{d_{j m}}-z_{d_{j m}}\right|: i \in \Lambda_{h}, j \in \Lambda_{K}\right\}\right\} \\
& \geq \max \left\{\left|L_{1}-L_{2}\right|-\sum_{m=\max \left\{T_{L_{1}}, T_{L_{2}}\right\}+\tau_{1}}^{\infty} t_{1 m} \max \{\|u-w\|,\|v-z\|\},\right. \\
& \left|L_{1}-L_{2}\right|-\sum_{m=\max }^{\infty}\left\{T_{\left.L_{1}, T_{L_{2}}\right\}+\tau_{2}}^{\infty} t_{2 m} \max \{\|u-w\|,\|v-z\|\}\right\} \\
& \geq\left|L_{1}-L_{2}\right|-\left(\sum_{m=\max \left\{T_{L_{1}}, T_{L_{2}}\right\}}^{\infty} \max \left\{t_{i m}: i \in \Lambda_{2}\right\}\right)\|(u, v)-(w, z)\|_{1} \\
& \geq\left|L_{1}-L_{2}\right|-\max \left\{\theta_{L_{1}}, \theta_{L_{2}}\right\}\|(u, v)-(w, z)\|_{1},
\end{aligned}
$$

which yields that

$$
\|(u, v)-(w, z)\|_{1} \geq \frac{\left|L_{1}-L_{2}\right|}{1+\max \left\{\theta_{L_{1}}, \theta_{L_{2}}\right\}}>0,
$$

that is, $(u, v) \neq(w, z)$. This completes the proof.

Theorem 2.3. Assume that there exist constants $M, N \in \mathbb{R}^{+} \backslash\{0\}, \bar{p}, p \in \mathbb{R}^{+}, n_{1} \in \mathbb{N}_{n_{0}}$, and four nonnegative sequences $\left\{r_{1 n}\right\}_{n \in \mathbb{N}_{n_{0}}},\left\{r_{2 n}\right\}_{n \in \mathbb{N}_{n_{0}}},\left\{t_{1 n}\right\}_{n \in \mathbb{N}_{n_{0}}}$, and $\left\{t_{2 n}\right\}_{n \in \mathbb{N}_{n_{0}}}$ satisfying (2.1), (2.2), (2.24), and

$$
N<(1-\underline{p}-\bar{p}) M, \quad \underline{p}+\bar{p}<1, \quad-\underline{p} \leq p_{\text {in }} \leq \bar{p}, \quad \forall n \geq n_{1}, i \in \Lambda_{2}
$$


Then,

(a) for each $L \in(N+\bar{p} M, M(1-p))$, there exist $\theta_{L} \in(0,1)$ and $T_{L} \geq \max \left\{\tau_{1}, \tau_{2}\right\}+n_{1}+|\alpha|$ such that for any $\left(x^{0}, y^{0}\right)=-\left(\left\{x_{n}^{0}\right\}_{n \in \mathbb{Z}_{\alpha}},\left\{y_{n}^{0}\right\}_{n \in \mathbb{Z}_{\alpha}}\right) \in A(N, M)$, the Mann iterative sequence with errors $\left\{\left(x^{\mu}, y^{\mu}\right)\right\}_{\mu \in \mathbb{N}_{0}}=\left\{\left(\left\{x_{n}^{\mu}\right\}_{n \in \mathbb{Z}_{\alpha}},\left\{y_{n}^{\mu}\right\}_{n \in \mathbb{Z}_{\alpha}}\right)\right\}_{\mu \in \mathbb{N}_{0}}$ generated by the schemes

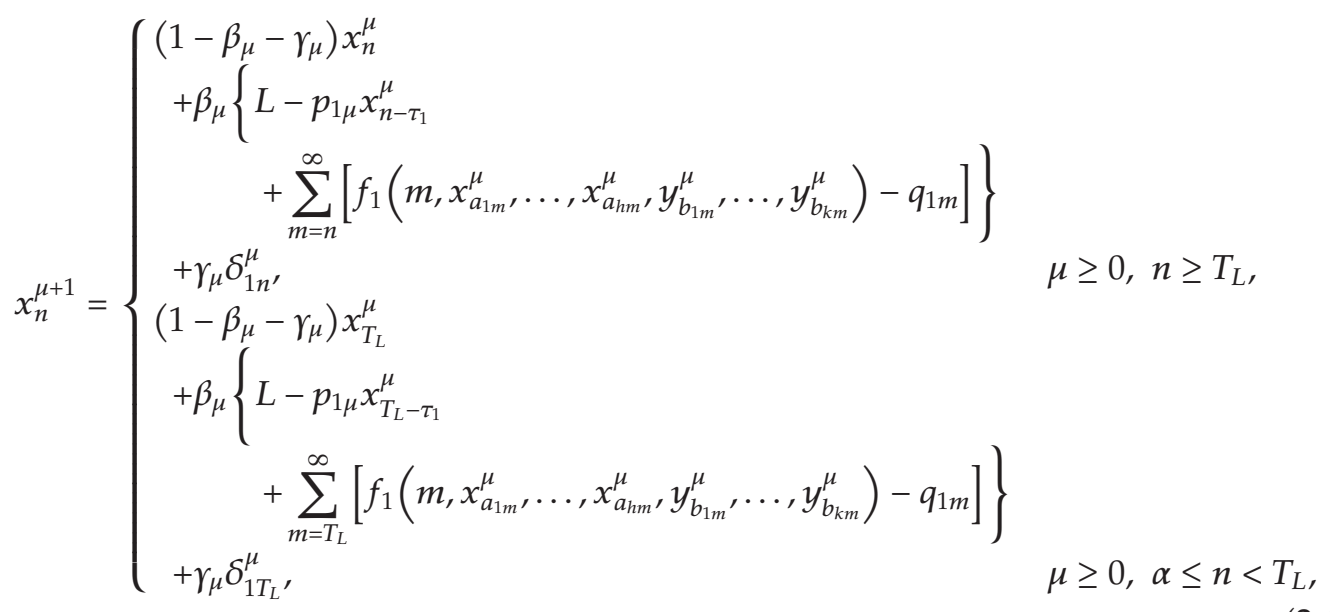

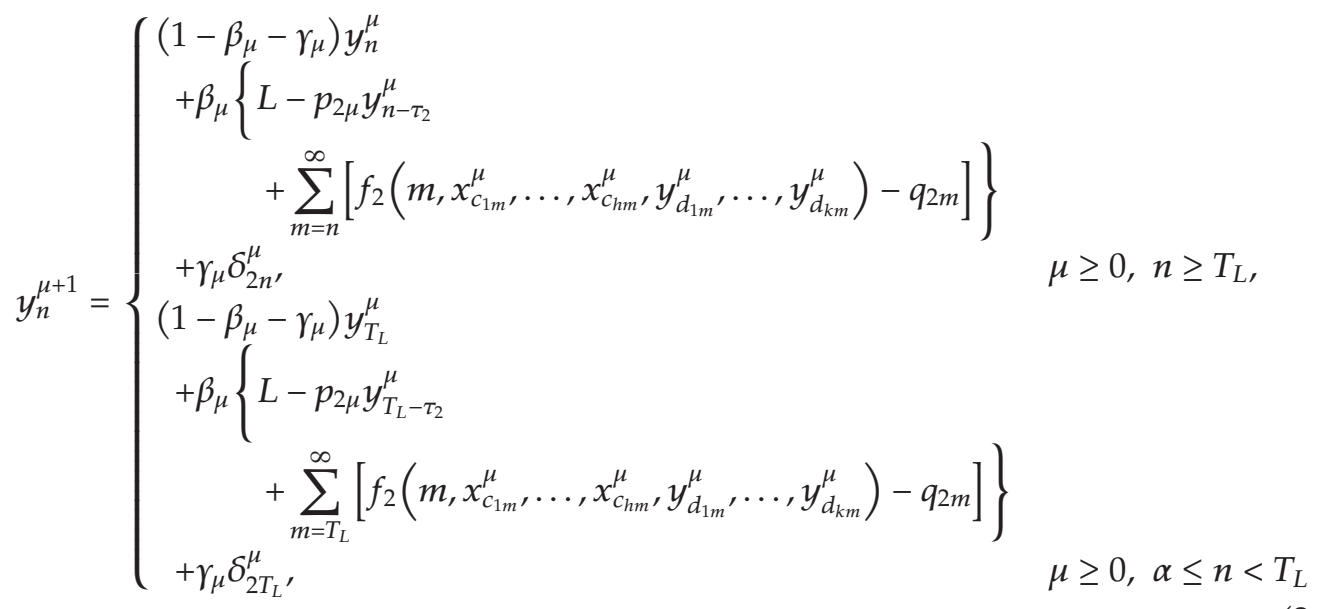

converges to a bounded positive solution $(x, y) \in A(N, M)$ of System (1.14) and has the error estimate (2.7), where $\left\{\beta_{\mu}\right\}_{\mu \in \mathbb{N}_{0}},\left\{\gamma_{\mu}\right\}_{\mu \in \mathbb{N}_{0}} \subset[0,1]$, and $\left\{\left(\delta_{1}^{\mu}, \delta_{2}^{\mu}\right)\right\}_{\mu \in \mathbb{N}_{0}}=\left\{\left(\left\{\delta_{1 n}^{\mu}\right\}_{n \in \mathbb{Z}_{\alpha^{\prime}}}\right.\right.$ $\left.\left.\left\{\delta_{2 n}^{\mu}\right\}_{n \in \mathbb{Z}_{\alpha}}\right)\right\}_{\mu \in \mathbb{N}_{0}} \subset A(N, M)$ are arbitrary sequences with (2.8) and (2.9),

(b) System (1.14) has uncountably many bounded positive solutions in $A(N, M)$.

Proof. (a) Let $L \in(N+\bar{p} M, M(1-p))$. Now we construct a contraction mapping $S_{L}$ : $A(N, M) \rightarrow A(N, M)$ and prove that its fixed point is a bounded positive solution of 
the system (1.14). Notice that (2.24) and (2.39) mean that there exist $\theta_{L} \in(0,1)$ and $T_{L} \geq$ $\max \left\{\tau_{1}, \tau_{2}\right\}+n_{1}+|\alpha|$ satisfying

$$
\begin{gathered}
\theta_{L}=\underline{p}+\bar{p}+\sum_{m=T_{L}}^{\infty} \max \left\{t_{i m}: i \in \Lambda_{2}\right\} \\
\sum_{m=T_{L}}^{\infty} \max \left\{r_{i m}+\left|q_{i m}\right|: i \in \Lambda_{2}\right\}<\min \{M(1-\underline{p})-L, L-N-\bar{p} M\} .
\end{gathered}
$$

Define three mappings $S_{L}: A(N, M) \rightarrow l_{\alpha}^{\infty} \times l_{\alpha}^{\infty}, S_{1 L}, S_{2 L}: A(N, M) \rightarrow l_{\alpha}^{\infty}$ by (2.14),

$$
\begin{aligned}
& S_{1 L}\left(x_{n}, y_{n}\right)= \begin{cases}L-p_{1 n} x_{n-\tau_{1}}+\sum_{m=n}^{\infty}\left[f_{1}\left(m, x_{a_{1 m}}, \ldots, x_{a_{h m}}, y_{b_{1 m}}, \ldots, y_{b_{k m}}\right)-q_{1 m}\right], & n \geq T_{L}, \\
S_{1 L}\left(x_{T_{L}}, y_{T_{L}}\right), & \alpha \leq n<T_{L},\end{cases} \\
& S_{2 L}\left(x_{n}, y_{n}\right)= \begin{cases}L-p_{2 n} y_{n-\tau_{2}}+\sum_{m=n}^{\infty}\left[f_{2}\left(m, x_{c_{1 m}}, \ldots, x_{c_{h m}}, y_{d_{1 m}}, \ldots, y_{d_{k m}}\right)-q_{2 m}\right], & n \geq T_{L}, \\
S_{2 L}\left(x_{T_{L}}, y_{T_{L}}\right), & \alpha \leq n<T_{L},\end{cases}
\end{aligned}
$$

for all $(x, y)=\left(\left\{x_{n}\right\}_{n \in \mathbb{Z}_{\alpha}},\left\{y_{n}\right\}_{n \in \mathbb{Z}_{\alpha}}\right) \in A(N, M)$.

It follows from (2.1), (2.2), (2.14), and (2.42)-(2.45) that for any $(x, y)=\left(\left\{x_{n}\right\}_{n \in \mathbb{Z}_{\alpha^{\prime}}}\right.$ $\left.\left\{y_{n}\right\}_{n \in \mathbb{Z}_{\alpha}}\right)$ and $(u, v)=\left(\left\{u_{n}\right\}_{n \in \mathbb{Z}_{\alpha}},\left\{v_{n}\right\}_{n \in \mathbb{Z}_{\alpha}}\right) \in A(N, M)$,

$$
\left.\begin{array}{l}
\left\|S_{L}(x, y)-S_{L}(u, v)\right\|_{1} \\
=\max \left\{\left\|S_{i L}(x, y)-S_{i L}(u, v)\right\|: i \in \Lambda_{2}\right\} \\
=\max \left\{\sup _{n \geq T_{L}}\left|S_{i L}\left(x_{n}, y_{n}\right)-S_{i L}\left(u_{n}, v_{n}\right)\right|: i \in \Lambda_{2}\right\} \\
\leq \max \left\{\operatorname { s u p } _ { n \geq T _ { L } } \left\{\left|p_{1 n}\left(x_{n-\tau_{1}}-u_{n-\tau_{1}}\right)\right|+\sum_{m=n}^{\infty} \mid f_{1}\left(m, x_{a_{1 m}}, \ldots, x_{a_{h m}}, y_{b_{1 m}}, \ldots, y_{b_{k m}}\right)\right.\right. \\
\left.\quad-f_{1}\left(m, u_{a_{1 m}}, \ldots, u_{a_{h m}}, v_{b_{1 m}}, \ldots, v_{b_{k m}}\right) \mid\right\}, \\
\sup _{n \geq T_{L}}\left\{\left|p_{2 n}\left(y_{n-\tau_{2}}-v_{n-\tau_{2}}\right)\right|+\sum_{m=n}^{\infty} \mid f_{2}\left(m, x_{c_{1 m}}, \ldots, x_{c_{h m}}, y_{d_{1 m}}, \ldots, y_{d_{k m}}\right)\right. \\
\leq \max \left\{(\underline{p}+\bar{p})\|x-u\|+\sum_{m=T_{L}}^{\infty} t_{1 m} \max \left\{\left|x_{a_{l m}}-u_{a_{l m}}\right|,\left|y_{b_{j m}}, \ldots, v_{b_{j m}}\right|: l \in \Lambda_{c_{h m}}, v_{d_{1 m}}, \ldots, v_{d_{k m}}\right) \mid\right\}
\end{array}\right\},
$$


Abstract and Applied Analysis

$$
\begin{aligned}
& \left.(\underline{p}+\bar{p})\|y-v\|+\sum_{m=T_{L}}^{\infty} t_{2 m} \max \left\{\left|x_{c_{l m}}-u_{c_{l m}}\right|,\left|y_{d_{j m}}-v_{d_{j m}}\right|: l \in \Lambda_{h}, j \in \Lambda_{k}\right\}\right\} \\
& \leq \max \left\{(\underline{p}+\bar{p})\|x-u\|+\sum_{m=T_{L}}^{\infty} t_{1 m} \max \{\|x-u\|,\|y-v\|\},\right. \\
& \left.(\underline{p}+\bar{p})\|y-v\|+\sum_{m=T_{L}}^{\infty} t_{2 m} \max \{\|x-u\|,\|y-v\|\}\right\} \\
& \leq\left(\underline{p}+\bar{p}+\sum_{m=T_{L}}^{\infty} \max \left\{t_{i m}: i \in \Lambda_{2}\right\}\right)\|(x, y)-(u, v)\|_{1} \\
& =\theta_{L}\|(x, y)-(u, v)\|_{1}, \\
& S_{1 L}\left(x_{n}, y_{n}\right) \\
& =L-p_{1 n} x_{n-\tau_{1}}+\sum_{m=n}^{\infty}\left[f_{1}\left(m, x_{a_{1 m}}, \ldots, x_{a_{h m}}, y_{b_{1 m}}, \ldots, y_{b_{k m}}\right)-q_{1 m}\right] \\
& \leq L+\underline{p} M+\sum_{m=T_{L}}^{\infty}\left(r_{1 m}+\left|q_{1 m}\right|\right) \\
& \leq L+\underline{p} M+\min \{M(1-\underline{p})-L, L-N-\bar{p} M\} \\
& \leq M, \quad \forall n \geq T_{L} \\
& S_{1 L}\left(x_{n}, y_{n}\right) \\
& =L-p_{1 n} x_{n-\tau_{1}}+\sum_{m=n}^{\infty}\left[f_{1}\left(m, x_{a_{1 m}}, \ldots, x_{a_{h m}}, y_{b_{1 m}}, \ldots, y_{b_{k m}}\right)-q_{1 m}\right] \\
& \geq L-\bar{p} M-\sum_{m=T_{L}}^{\infty}\left(r_{1 m}+\left|q_{1 m}\right|\right) \\
& \geq L-\bar{p} M-\min \{M(1-\underline{p})-L, L-N-\bar{p} M\} \\
& \geq N, \quad \forall n \geq T_{L}, \\
& N \leq S_{2 L}\left(x_{n}, y_{n}\right) \leq M, \quad \forall n \geq T_{L}
\end{aligned}
$$

which give (2.16) and (2.17), which together with $\theta_{L} \in(0,1)$ guarantee that $S_{L}: A(N, M) \rightarrow$ $A(N, M)$ is a contraction. Thus the Banach fixed point theorem ensures that $S_{L}$ has a unique fixed point $(x, y)=\left(\left\{x_{n}\right\}_{n \in \mathbb{Z}_{\alpha}}\left\{y_{n}\right\}_{n \in \mathbb{Z}_{\alpha}}\right) \in A(N, M)$, that is,

$$
\begin{gathered}
x_{n}=L-p_{1 n} x_{n-\tau_{1}}+\sum_{m=n}^{\infty}\left[f_{1}\left(m, x_{a_{1 m}}, \ldots, x_{a_{h m}}, y_{b_{1 m}}, \ldots, y_{b_{k m}}\right)-q_{1 m}\right], \\
y_{n}=L-p_{2 n} y_{n-\tau_{2}}+\sum_{m=n}^{\infty}\left[f_{2}\left(m, x_{c_{1 m}}, \ldots, x_{c_{h m}}, y_{d_{1 m}}, \ldots, y_{d_{k m}}\right)-q_{2 m}\right], \quad n \geq T_{L},
\end{gathered}
$$


which yield that

$$
\begin{gathered}
\Delta\left(x_{n}+p_{1 n} x_{n-\tau_{1}}\right)=-f_{1}\left(n, x_{a_{1 n}}, \ldots, x_{a_{h n}}, y_{b_{1 n}}, \ldots, y_{b_{k n}}\right)+q_{1 n}, \\
\Delta\left(y_{n}+p_{2 n} y_{n-\tau_{2}}\right)=f_{2}\left(n, x_{c_{1 n}}, \ldots, x_{c_{h n}}, y_{d_{1 n}}, \ldots, y_{d_{k n}}\right)+q_{2 n}, \quad \forall n \geq T_{L},
\end{gathered}
$$

that is, $(x, y)$ is a bounded positive solution of System (1.14) in $A(N, M)$.

In light of (2.14), (2.16), (2.40), (2.41), (2.44), and (2.45), we deduce that

$$
\begin{aligned}
& \left\|\left(x^{\mu+1}, y^{\mu+1}\right)-(x, y)\right\|_{1} \\
& =\max \left\{\sup _{n \geq T_{L}}\left|x_{n}^{\mu+1}-x_{n}\right|, \sup _{n \geq T_{L}}\left|y_{n}^{\mu+1}-y_{n}\right|\right\} \\
& \leq \max \left\{\operatorname { s u p } _ { n \geq T _ { L } } \left[\left(1-\beta_{\mu}-\gamma_{\mu}\right)\left|x_{n}^{\mu}-x_{n}\right|\right.\right. \\
& +\beta_{\mu}\left|L-p_{1 n} x_{n-\tau_{1}}+\sum_{m=n}^{\infty}\left[f_{1}\left(m, x_{a_{1 m}}^{\mu}, \ldots, x_{a_{h m}}^{\mu}, y_{b_{1 m}}^{\mu}, \ldots, y_{b_{k m}}^{\mu}\right)-q_{1 m}\right]-x_{n}\right| \\
& \left.+\gamma_{\mu}\left|\delta_{1 n}^{\mu}-x_{n}\right|\right] \\
& \sup _{n \geq T_{L}}\left[\left(1-\beta_{\mu}-\gamma_{\mu}\right)\left|y_{n}^{\mu}-y_{n}\right|\right. \\
& +\beta_{\mu}\left|L-p_{2 n} y_{n-\tau_{2}}+\sum_{m=n}^{\infty}\left[f_{2}\left(m, x_{c_{1 m}}^{\mu}, \ldots, x_{c_{h m}}^{\mu}, y_{d_{1 m}}^{\mu}, \ldots, y_{d_{k m}}^{\mu}\right)-q_{2 m}\right]-y_{n}\right| \\
& \left.\left.+r_{\mu}\left|\delta_{2 n}^{\mu}-y_{n}\right|\right]\right\} \\
& \leq \max \left\{\sup _{n \geq T_{L}}\left[\left(1-\beta_{\mu}-\gamma_{\mu}\right)\left\|x^{\mu}-x\right\|+\beta_{\mu}\left|S_{1 L}\left(x_{n}^{\mu}, y_{n}^{\mu}\right)-S_{1 L}\left(x_{n}, y_{n}\right)\right|+2 M \gamma_{\mu}\right]\right. \text {, } \\
& \left.\sup _{n \geq T_{L}}\left[\left(1-\beta_{\mu}-\gamma_{\mu}\right)\left\|y^{\mu}-y\right\|+\beta_{\mu}\left|S_{2 L}\left(x_{n}^{\mu}, y_{n}^{\mu}\right)-S_{2 L}\left(x_{n}, y_{n}\right)\right|+2 M \gamma_{\mu}\right]\right\} \\
& \leq\left(1-\beta_{\mu}\left(1-\theta_{L}\right)\right)\left\|\left(x^{\mu}, y^{\mu}\right)-(x, y)\right\|_{1}+2 M \gamma_{\mu}, \quad \forall \mu \geq 0,
\end{aligned}
$$

which implies (2.7). Thus Lemma 1.1 and (2.7)-(2.9) imply that $\lim _{\mu \rightarrow \infty}\left\|\left(x^{\mu}, y^{\mu}\right)-(x, y)\right\|_{1}=$ 0 . 
(b) Let $L_{1}, L_{2} \in(N, M)$ and $L_{1} \neq L_{2}$. Similar to the proof of (a), we know that for each $i \in \Lambda_{2}$, there exist a constant $\theta_{L_{i}} \in(0,1)$, a positive integer $T_{L_{i}} \geq \max \left\{\tau_{1}, \tau_{2}\right\}+n_{1}+|\alpha|$ and mappings $S_{L_{i}}, S_{1 L_{i}}, S_{2 L_{i}}$ satisfying (2.14) and (2.42)-(2.45), where $L, \theta_{L}$, and $T_{L}$ are replaced by $L_{i}, \theta_{L_{i}}$ and $T_{L_{i}}$, respectively, and the contraction mappings $S_{L_{1}}$ and $S_{L_{2}}$ have the unique fixed points $(u, v)=\left(\left\{u_{n}\right\}_{n \in \mathbb{Z}_{\alpha}},\left\{v_{n}\right\}_{n \in \mathbb{Z}_{\alpha}}\right),(w, z)=\left(\left\{w_{n}\right\}_{n \in \mathbb{Z}_{\alpha}},\left\{z_{n}\right\}_{n \in \mathbb{Z}_{\alpha}}\right) \in A(N, M)$, which are bounded positive solutions of System (1.14) in $A(N, M)$, respectively. In order to show that System (1.14) possesses uncountably many bounded positive solutions in $A(N, M)$, we prove only that $(u, v) \neq(w, z)$. In terms of $(2.14)$ and (2.42)-(2.45), we get that

$$
\begin{aligned}
& \|(u, v)-(w, z)\|_{1} \\
& =\max \{\|u-w\|,\|v-z\|\} \\
& \geq \max \left\{\sup _{n \geq \max \left\{T_{L_{1}}, T_{L_{2}}\right\}}\left|u_{n}-w_{n}\right|, \sup _{n \geq \max \left\{T_{L_{1}}, T_{L_{2}}\right\}}\left|v_{n}-z_{n}\right|\right\} \\
& \geq \max \left\{\operatorname { s u p } _ { n \geq \operatorname { m a x } \{ T _ { L _ { 1 } } , T _ { L _ { 2 } } \} } \left\{\left|L_{1}-L_{2}\right|-\left|p_{1 n}\right|\left|u_{n-\tau_{1}}-w_{n-\tau_{1}}\right|\right.\right. \\
&
\end{aligned}
$$




$$
\begin{gathered}
-\sum_{m=\max \left\{T_{\left.L_{1}, T_{L_{2}}\right\}} t_{2 m} \max \left\{\left|u_{c_{i m}}-w_{c_{i m}}\right|,\left|v_{d_{j m}}-z_{d_{j m}}\right|: i \in \Lambda_{h}, j \in \Lambda_{K}\right\}\right\}} \geq \max \left\{\left|L_{1}-L_{2}\right|-(\underline{p}+\bar{p})\|u-w\|-\sum_{m=\max \left\{T_{L_{1}}, T_{L_{2}}\right\}}^{\infty} t_{1 m} \max \{\|u-w\|,\|v-z\|\},\right. \\
\left.\left|L_{1}-L_{2}\right|-(\underline{p}+\bar{p})\|v-z\|-\sum_{m=\max \left\{T_{L_{1}}, T_{L_{2}}\right\}}^{\infty} t_{2 m} \max \{\|u-w\|,\|v-z\|\}\right\} \\
\geq\left|L_{1}-L_{2}\right|-\left(\underline{p}+\bar{p}+\sum_{m=\max \left\{T_{L_{1}}, T_{L_{2}}\right\}}^{\infty} \max \left\{t_{i m}: i \in \Lambda_{2}\right\}\right)\|(u, v)-(w, z)\|_{1}
\end{gathered}
$$

which yields that

$$
\|(u, v)-(w, z)\|_{1} \geq \frac{\left|L_{1}-L_{2}\right|}{1+\max \left\{\theta_{L_{1}}, \theta_{L_{2}}\right\}}>0,
$$

that is, $(u, v) \neq(w, z)$. This completes the proof.

Theorem 2.4. Assume that there exist constants $M, N, \bar{p}, \underline{p} \in \mathbb{R}^{+} \backslash\{0\}, n_{1} \in \mathbb{N}_{n_{0}}$ and four nonnegative sequences $\left\{r_{1 n}\right\}_{n \in \mathbb{N}_{n_{0}}},\left\{r_{2 n}\right\}_{n \in \mathbb{N}_{n_{0}}},\left\{t_{1 n}\right\}_{n \in \mathbb{N}_{n_{0}}}$ and $\left\{t_{2 n}\right\}_{n \in \mathbb{N}_{n_{0}}}$ satisfying (2.1), (2.2), (2.24), and

$$
0<N(\underline{p}-1)<M(\bar{p}-1), \quad-\underline{p} \leq p_{\text {in }} \leq-\bar{p}, \quad \forall n \geq n_{1}, i \in \Lambda_{2}
$$

Then,

(a) for each $L \in(N(\underline{p}-1), M(\bar{p}-1))$, there exist $\theta_{L} \in(0,1)$ and $T_{L} \geq \max \left\{\tau_{1}, \tau_{2}\right\}+n_{1}+|\alpha|$ such that for any $\left(x^{0}, y^{0}\right)=\left(\left\{x_{n}^{0}\right\}_{n \in \mathbb{Z}_{\alpha}},\left\{y_{n}^{0}\right\}_{n \in \mathbb{Z}_{\alpha}}\right) \in A(N, M)$, the Mann iterative 
sequence with errors $\left\{\left(x^{\mu}, y^{\mu}\right)\right\}_{\mu \in \mathbb{N}_{0}}=\left\{\left(\left\{x_{n}^{\mu}\right\}_{n \in \mathbb{Z}_{\alpha^{\prime}}},\left\{y_{n}^{\mu}\right\}_{n \in \mathbb{Z}_{\alpha}}\right)\right\}_{\mu \in \mathbb{N}_{0}}$ generated by the schemes

$$
\begin{aligned}
& \left\{\begin{array}{l}
\left(1-\beta_{\mu}-\gamma_{\mu}\right) x_{n}^{\mu} \\
+\beta_{\mu}\left\{\frac{-L}{p_{1 n+\tau_{1}}}-\frac{x_{n+\tau_{1}}^{\mu}}{p_{1 n+\tau_{1}}}+\frac{1}{p_{1 n+\tau_{1}}}\right.
\end{array}\right. \\
& x_{n}^{\mu+1}=\left\{\begin{array}{cc}
\left.\times \sum_{m=n+\tau_{1}}^{\infty}\left[f_{1}\left(m, x_{a_{1 m}}^{\mu}, \ldots, x_{a_{h m}}^{\mu}, y_{b_{1 m^{\prime}}}^{\mu}, \ldots, y_{b_{k m}}^{\mu}\right)-q_{1 m}\right]\right\} & \\
+\gamma_{\mu} \delta_{1 n^{\prime}}^{\mu} & \mu \geq 0, n \geq T_{L}, \\
\left(1-\beta_{\mu}-\gamma_{\mu}\right) x_{T_{L}}^{\mu} &
\end{array}\right. \\
& +\beta_{\mu}\left\{\frac{-L}{p_{1 T_{L}+\tau_{1}}}-\frac{x_{T_{L}+\tau_{1}}^{\mu}}{p_{1 T_{L}+\tau_{1}}}+\frac{1}{p_{1 T_{L}+\tau_{1}}}\right. \\
& \left.\times \sum_{m=T_{L}+\tau_{1}}^{\infty}\left[f_{1}\left(m, x_{a_{1 m}}^{\mu}, \ldots, x_{a_{h m}}^{\mu}, y_{b_{1 m}}^{\mu}, \ldots, y_{b_{k m}}^{\mu}\right)-q_{1 m}\right]\right\} \\
& +\gamma_{\mu} \delta_{1 T_{L},}^{\mu} \quad \mu \geq 0, \alpha \leq n<T_{L},
\end{aligned}
$$

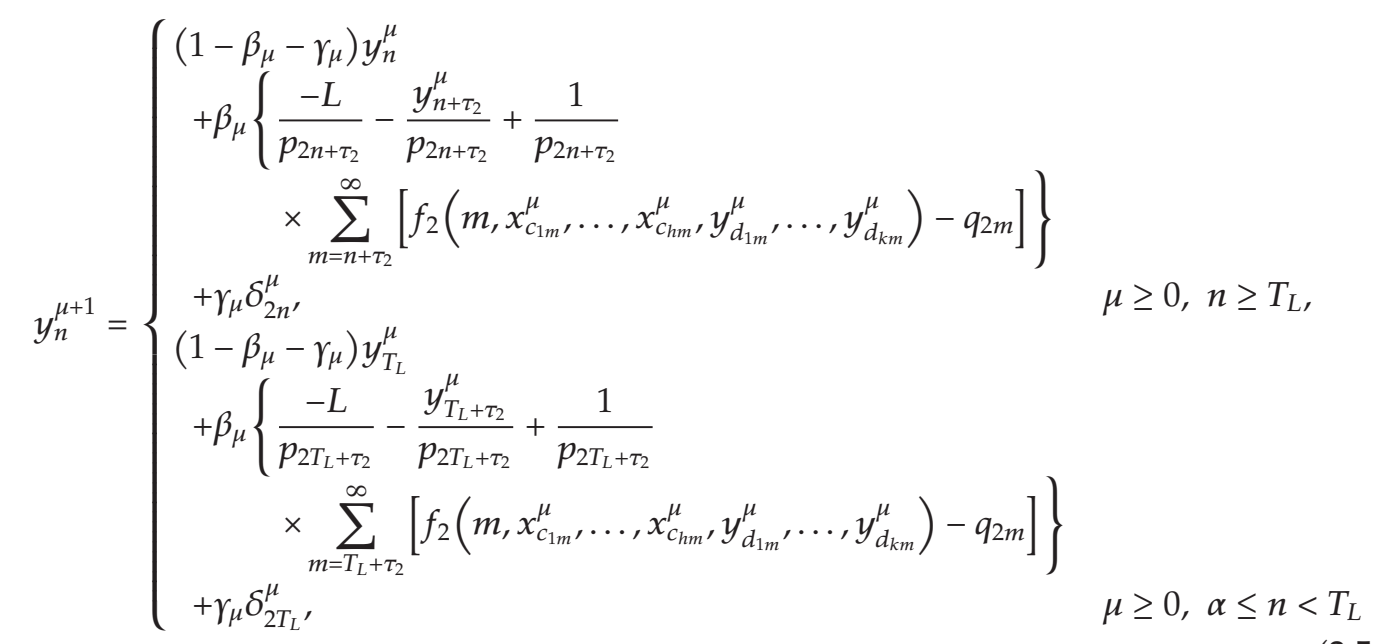

converges to a bounded positive solution $(x, y) \in A(N, M)$ of System (1.14) and has the error estimate (2.7), where $\left\{\beta_{\mu}\right\}_{\mu \in \mathbb{N}_{0}},\left\{\gamma_{\mu}\right\}_{\mu \in \mathbb{N}_{0}} \subset[0,1]$ and $\left\{\left(\delta_{1}^{\mu}, \delta_{2}^{\mu}\right)\right\}_{\mu \in \mathbb{N}_{0}}=$ $\left\{\left(\left\{\delta_{1 n}^{\mu}\right\}_{n \in \mathbb{Z}_{\alpha}}\left\{\delta_{2 n}^{\mu}\right\}_{n \in \mathbb{Z}_{\alpha}}\right)\right\}_{\mu \in \mathbb{N}_{0}} \subset A(N, M)$ are arbitrary sequences with (2.8) and (2.9),

(b) System (1.14) has uncountably many bounded positive solutions in $A(N, M)$.

Proof. (a) Let $L \in(N(p-1), M(\bar{p}-1))$. Now we construct a contraction mapping $S_{L}$ : $A(N, M) \rightarrow A(N, M)$ and prove that its fixed point is a bounded positive solution of 
the system (1.14). Observe that (2.24) and (2.52) guarantee that there exist $\theta_{L} \in(0,1)$ and $T_{L} \geq \max \left\{\tau_{1}, \tau_{2}\right\}+n_{1}+|\alpha|$ satisfying

$$
\begin{gathered}
\theta_{L}=\frac{1}{\bar{p}}\left(1+\sum_{m=T_{L}}^{\infty} \max \left\{t_{i m}: i \in \Lambda_{2}\right\}\right), \\
\sum_{m=T_{L}}^{\infty} \max \left\{r_{i m}+\left|q_{i m}\right|: i \in \Lambda_{2}\right\}<\min \left\{M(\bar{p}-1)-L, \bar{p}\left(\frac{L+N}{\underline{p}}-N\right)\right\} .
\end{gathered}
$$

Define three mappings $S_{L}: A(N, M) \rightarrow l_{\alpha}^{\infty} \times l_{\alpha}^{\infty}, S_{1 L}, S_{2 L}: A(N, M) \rightarrow l_{\alpha}^{\infty}$ by (2.14),

$$
\begin{gathered}
S_{1 L}\left(x_{n}, y_{n}\right)= \begin{cases}\frac{-L}{p_{1 n+\tau_{1}}}-\frac{x_{n+\tau_{1}}}{p_{1 n+\tau_{1}}} & \\
+\frac{1}{p_{1 n+\tau_{1}}} \sum_{m=n+\tau_{1}}^{\infty}\left[f_{1}\left(m, x_{a_{1 m}}, \ldots, x_{a_{h m}}, y_{b_{1 m}}, \ldots, y_{b_{k m}}\right)-q_{1 m}\right], & n \geq T_{L}, \\
S_{1 L}\left(x_{T_{L}}, y_{T_{L}}\right), & \alpha \leq n<T_{L},\end{cases} \\
S_{2 L}\left(x_{n}, y_{n}\right)= \begin{cases}\frac{-L}{p_{2 n+\tau_{2}}}-\frac{y_{n+\tau_{2}}}{p_{2 n+\tau_{2}}} & \\
+\frac{1}{p_{2 n+\tau_{2}}} \sum_{m=n+\tau_{2}}^{\infty}\left[f_{2}\left(m, x_{c_{1 m}}, \ldots, x_{c_{h m}}, y_{d_{1 m}}, \ldots, y_{d_{k m}}\right)-q_{2 m}\right], & n \geq T_{L}, \\
S_{2 L}\left(x_{T_{L}}, y_{T_{L}}\right), & \alpha \leq n<T_{L},\end{cases}
\end{gathered}
$$

for all $(x, y)=\left(\left\{x_{n}\right\}_{n \in \mathbb{Z}_{\alpha}},\left\{y_{n}\right\}_{n \in \mathbb{Z}_{\alpha}}\right) \in A(N, M)$. 
It follows from (2.1), (2.2), (2.14), and (2.55)-(2.58) that for any $(x, y)=\left(\left\{x_{n}\right\}_{n \in \mathbb{Z}_{\alpha^{\prime}}}\right.$ $\left.\left\{y_{n}\right\}_{n \in \mathbb{Z}_{\alpha}}\right)$ and $(u, v)=\left(\left\{u_{n}\right\}_{n \in \mathbb{Z}_{\alpha}}\left\{v_{n}\right\}_{n \in \mathbb{Z}_{\alpha}}\right) \in A(N, M)$

$$
\begin{aligned}
& \left\|S_{L}(x, y)-S_{L}(u, v)\right\|_{1} \\
& =\max \left\{\left\|S_{i L}(x, y)-S_{i L}(u, v)\right\|: i \in \Lambda_{2}\right\} \\
& =\max \left\{\sup _{n \geq T_{L}}\left|S_{i L}\left(x_{n}, y_{n}\right)-S_{i L}\left(u_{n}, v_{n}\right)\right|: i \in \Lambda_{2}\right\} \\
& \leq \max \left\{\operatorname { s u p } _ { n \geq T _ { L } } \left\{\frac { 1 } { | p _ { 1 n + \tau _ { 1 } } | } \left(\left|x_{n+\tau_{1}}-u_{n+\tau_{1}}\right|\right.\right.\right. \\
& +\sum_{m=n+\tau_{1}}^{\infty} \mid f_{1}\left(m, x_{a_{1 m}}, \ldots, x_{a_{h m}}, y_{b_{1 m}}, \ldots, y_{b_{k m}}\right) \\
& \left.\left.-f_{1}\left(m, u_{a_{1 m}}, \ldots, u_{a_{h m}}, v_{b_{1 m}}, \ldots, v_{b_{k m}}\right) \mid\right)\right\} \\
& \sup _{n \geq T_{L}}\left\{\frac { 1 } { | p _ { 2 n + \tau _ { 2 } } | } \left(\left|y_{n+\tau_{2}}-v_{n+\tau_{2}}\right|\right.\right. \\
& +\sum_{m=n+\tau_{2}}^{\infty} \mid f_{2}\left(m, x_{c_{1 m}}, \ldots, x_{c_{h m}}, y_{d_{1 m}}, \ldots, y_{d_{k m}}\right) \\
& \left.\left.\left.-f_{2}\left(m, u_{c_{1 m}}, \ldots, u_{c_{h m}}, v_{d_{1 m}}, \ldots, v_{d_{k m}}\right) \mid\right)\right\}\right\} \\
& \leq \frac{1}{\bar{p}} \max \left\{\|x-u\|+\sum_{m=T_{L}}^{\infty} t_{1 m} \max \left\{\left|x_{a_{i m}}-u_{a_{i m}}\right|,\left|y_{b_{j m}}-v_{b_{j m}}\right|: i \in \Lambda_{h}, j \in \Lambda_{k}\right\},\right. \\
& \left.\|y-v\|+\sum_{m=T_{L}}^{\infty} t_{2 m} \max \left\{\left|x_{c_{i m}}-u_{c_{i m}}\right|,\left|y_{d_{j m}}-v_{d_{j m}}\right|: i \in \Lambda_{h}, j \in \Lambda_{k}\right\}\right\} \\
& \leq \frac{1}{\bar{p}} \max \left\{\|x-u\|+\sum_{m=T_{L}}^{\infty} t_{1 m} \max \{\|x-u\|,\|y-v\|\},\right. \\
& \left.\|y-v\|+\sum_{m=T_{L}}^{\infty} t_{2 m} \max \{\|x-u\|,\|y-v\|\}\right\} \\
& \leq \frac{1}{\bar{p}}\left(1+\sum_{m=T_{L}}^{\infty} \max \left\{t_{i m}: i \in \Lambda_{2}\right\}\right)\|(x, y)-(u, v)\|_{1} \\
& =\theta_{L}\|(x, y)-(u, v)\|_{1},
\end{aligned}
$$




$$
\begin{aligned}
& S_{1 L}(\left.x_{n}, y_{n}\right) \\
&=\frac{-L}{p_{1 n+\tau_{1}}}-\frac{x_{n+\tau_{1}}}{p_{1 n+\tau_{1}}}+\frac{1}{p_{1 n+\tau_{1}}} \sum_{m=n+\tau_{1}}^{\infty}\left[f_{1}\left(m, x_{a_{1 m}}, \ldots, x_{a_{h m}}, y_{b_{1 m}}, \ldots, y_{b_{k m}}\right)-q_{1 m}\right] \\
& \leq \frac{L}{\bar{p}}+\frac{M}{\bar{p}}+\frac{1}{\bar{p}} \sum_{m=T_{L}+\tau_{1}}^{\infty}\left(r_{1 m}+\left|q_{1 m}\right|\right) \\
& \leq \frac{L}{\bar{p}}+\frac{M}{\bar{p}}+\frac{1}{\bar{p}} \min \left\{M(\bar{p}-1)-L, \bar{p}\left(\frac{L+N}{\underline{p}}-N\right)\right\} \\
& \leq M, \quad \forall n \geq T_{L}, \\
& S_{1 L}\left(x_{n}, y_{n}\right) \frac{-L}{p_{1 n+\tau_{1}}}-\frac{x_{n+\tau_{1}}}{p_{1 n+\tau_{1}}}+\frac{1}{p_{1 n+\tau_{1}}} \sum_{m=n+\tau_{1}}^{\infty}\left[f_{1}\left(m, x_{a_{1 m}}, \ldots, x_{a_{h m}}, y_{b_{1 m}}, \ldots, y_{b_{k m}}\right)-q_{1 m}\right] \\
& \quad \geq \frac{L}{\underline{p}}+\frac{N}{\underline{p}}-\frac{1}{\bar{p}} \sum_{m=T_{L}+\tau_{1}}^{\infty}\left(r_{1 m}+\left|q_{1 m}\right|\right) \\
& \geq \frac{L}{\underline{p}}+\frac{N}{\underline{p}}-\frac{1}{\bar{p}} \min \left\{M(\bar{p}-1)-L, \bar{p}\left(\frac{L+N}{\underline{p}}-N\right)\right\} \\
& \geq \\
& \quad N, \quad \forall n \geq T_{L}, \\
& N \leq S_{2 L}\left(x_{n}, y_{n}\right) \leq M, \quad \forall n \geq T_{L},
\end{aligned}
$$

which yield (2.16) and (2.17), which together with $\theta_{L} \in(0,1)$ ensure that $S_{L}$ is a contraction in $A(N, M)$. By the Banach fixed point theorem, we deduce that $S_{L}$ has a unique fixed point $(x, y)=\left(\left\{x_{n}\right\}_{n \in \mathbb{Z}_{\alpha}},\left\{y_{n}\right\}_{n \in \mathbb{Z}_{\alpha}}\right) \in A(N, M)$, that is,

$$
\begin{array}{ll}
x_{n}=\frac{-L}{p_{1 n+\tau_{1}}}-\frac{x_{n+\tau_{1}}}{p_{1 n+\tau_{1}}}+\frac{1}{p_{1 n+\tau_{1}}} \sum_{m=n+\tau_{1}}^{\infty}\left[f_{1}\left(m, x_{a_{1 m}}, \ldots, x_{a_{h m}}, y_{b_{1 m}}, \ldots, y_{b_{k m}}\right)-q_{1 m}\right], \quad n \geq T_{L}, \\
y_{n}=\frac{-L}{p_{2 n+\tau_{2}}}-\frac{y_{n+\tau_{2}}}{p_{2 n+\tau_{2}}}+\frac{1}{p_{2 n+\tau_{2}}} \sum_{m=n+\tau_{2}}^{\infty}\left[f_{2}\left(m, x_{c_{1 m}}, \ldots, x_{c_{h m}}, y_{d_{1 m}}, \ldots, y_{d_{k m}}\right)-q_{2 m}\right], \quad n \geq T_{L},
\end{array}
$$

which yield that

$$
\begin{array}{ll}
x_{n}+p_{1 n} x_{n-\tau_{1}}=-L+\sum_{m=n}^{\infty}\left[f_{1}\left(m, x_{a_{1 m}}, \ldots, x_{a_{h m}}, y_{b_{1 m}}, \ldots, y_{b_{k m}}\right)-q_{1 m}\right], & n \geq T_{L}+\tau_{1}, \\
y_{n}+p_{2 n} y_{n-\tau_{2}}=-L+\sum_{m=n}^{\infty}\left[f_{2}\left(m, x_{c_{1 m}}, \ldots, x_{c_{h m}}, y_{d_{1 m}}, \ldots, y_{d_{k m}}\right)-q_{2 m}\right], & n \geq T_{L}+\tau_{2},
\end{array}
$$


Abstract and Applied Analysis

$$
\begin{array}{ll}
\Delta\left(x_{n}+p_{1 n} x_{n-\tau_{1}}\right)=-f_{1}\left(n, x_{a_{1 n}}, \ldots, x_{a_{h n}}, y_{b_{1 n}}, \ldots, y_{b_{k n}}\right)+q_{1 n}, & \forall n \geq T_{L}+\tau_{1}, \\
\Delta\left(y_{n}+p_{2 n} y_{n-\tau_{2}}\right)=-f_{2}\left(n, x_{c_{1 n}}, \ldots, x_{c_{h n}}, y_{d_{1 n}}, \ldots, y_{d_{k n}}\right)+q_{2 n}, & \forall n \geq T_{L}+\tau_{2},
\end{array}
$$

that is, $(x, y)$ is a bounded positive solution of the system (1.14) in $A(N, M)$.

In light of (2.14), (2.16), (2.53)-(2.55), (2.57), and (2.58), we get that

$$
\begin{aligned}
& \left\|\left(x^{\mu+1}, y^{\mu+1}\right)-(x, y)\right\|_{1} \\
& =\max \left\{\sup _{n \geq T_{L}}\left|x_{n}^{\mu+1}-x_{n}\right|, \sup _{n \geq T_{L}}\left|y_{n}^{\mu+1}-y_{n}\right|\right\} \\
& \leq \max \left\{\operatorname { s u p } _ { n \geq T _ { L } } \left[\left(1-\beta_{\mu}-\gamma_{\mu}\right)\left|x_{n}^{\mu}-x_{n}\right|+\beta_{\mu}\right.\right. \\
& \times\left|\frac{-L}{p_{1 n+\tau_{1}}}-\frac{x_{n+\tau_{1}}^{\mu}}{p_{1 n+\tau_{1}}}+\frac{1}{p_{1 n+\tau_{1}}} \sum_{m=n}^{\infty}\left[f_{1}\left(m, x_{a_{1 m}}^{\mu}, \ldots, x_{a_{h m}}^{\mu}, y_{b_{1 m}}^{\mu}, \ldots, y_{b_{k m}}^{\mu}\right)-q_{1 m}\right]-x_{n}\right| \\
& \left.+\gamma_{\mu}\left|\delta_{1 n}^{\mu}-x_{n}\right|\right] \\
& \sup _{n \geq T_{L}}\left[\left(1-\beta_{\mu}-\gamma_{\mu}\right)\left|y_{n}^{\mu}-y_{n}\right|+\beta_{\mu}\right. \\
& \times\left|\frac{-L}{p_{2 n+\tau_{2}}}-\frac{y_{n+\tau_{2}}^{\mu}}{p_{2 n+\tau_{2}}}+\frac{1}{p_{2 n+\tau_{2}}} \sum_{m=n}^{\infty}\left[f_{2}\left(m, x_{c_{1 m}}^{\mu}, \ldots, x_{c_{h m}}^{\mu}, y_{d_{1 m}}^{\mu}, \ldots, y_{d_{k m}}^{\mu}\right)-q_{2 m}\right]-y_{n}\right| \\
& \left.\left.+\gamma_{\mu}\left|\delta_{2 n}^{\mu}-y_{n}\right|\right]\right\} \\
& \leq \max \left\{\sup _{n \geq T_{L}}\left[\left(1-\beta_{\mu}-\gamma_{\mu}\right)\left\|x^{\mu}-x\right\|+\beta_{\mu}\left|S_{1 L}\left(x_{n}^{\mu}, y_{n}^{\mu}\right)-S_{1 L}\left(x_{n}, y_{n}\right)\right|+2 M \gamma_{\mu}\right]\right. \text {, } \\
& \left.\sup _{n \geq T_{L}}\left[\left(1-\beta_{\mu}-\gamma_{\mu}\right)\left\|y^{\mu}-y\right\|+\beta_{\mu}\left|S_{2 L}\left(x_{n}^{\mu}, y_{n}^{\mu}\right)-S_{2 L}\left(x_{n}, y_{n}\right)\right|+2 M \gamma_{\mu}\right]\right\} \\
& \leq\left(1-\beta_{\mu}\left(1-\theta_{L}\right)\right)\left\|\left(x^{\mu}, y^{\mu}\right)-(x, y)\right\|_{1}+2 M \gamma_{\mu}, \quad \forall \mu \geq 0 \text {, }
\end{aligned}
$$

which implies (2.7). Thus Lemma 1.1 and (2.7)-(2.9) imply that $\lim _{\mu \rightarrow \infty}\left\|\left(x^{\mu}, y^{\mu}\right)-(x, y)\right\|_{1}=$ 0 .

(b) Let $L_{1}, L_{2} \in(N, M)$ and $L_{1} \neq L_{2}$. Similar to the proof of (a), we conclude that for each $i \in \Lambda_{2}$, there exist a constant $\theta_{L_{i}} \in(0,1)$, a positive integer $T_{L_{i}} \geq \max \left\{\tau_{1}, \tau_{2}\right\}+n_{1}+|\alpha|$, and mappings $S_{L_{i}}, S_{1 L_{i}}, S_{2 L_{i}}$ satisfying (2.14) and (2.55)-(2.58), where $L, \theta_{L}$, and $T_{L}$ are replaced by $L_{i}, \theta_{L_{i}}$, and $T_{L_{i}}$, respectively, and the contraction mappings $S_{L_{1}}$ and $S_{L_{2}}$ have the unique fixed points $(u, v)=\left(\left\{u_{n}\right\}_{n \in \mathbb{Z}_{\alpha}},\left\{v_{n}\right\}_{n \in \mathbb{Z}_{\alpha}}\right),(w, z)=\left(\left\{w_{n}\right\}_{n \in \mathbb{Z}_{\alpha}},\left\{z_{n}\right\}_{n \in \mathbb{Z}_{\alpha}}\right) \in A(N, M)$, which 
are bounded positive solutions of System (1.14) in $A(N, M)$, respectively. In order to show that System (1.14) possesses uncountably many bounded positive solutions in $A(N, M)$, we prove only that $(u, v) \neq(w, z)$. By virtue of $(2.14)$ and (2.55)-(2.58), we get that

$$
\begin{aligned}
& \|(u, v)-(w, z)\|_{1} \\
& =\max \{\|u-w\|,\|v-z\|\} \\
& \geq \max \left\{\sup _{n \geq \max \left\{T_{L_{1}}, T_{L_{2}}\right\}}\left|u_{n}-w_{n}\right|, \sup _{n \geq \max \left\{T_{L_{1}}, T_{L_{2}}\right\}}\left|v_{n}-z_{n}\right|\right\} \\
& \geq \max \left\{\operatorname { s u p } _ { n \geq \operatorname { m a x } \{ T _ { L _ { 1 } } , T _ { L _ { 2 } } \} } \left\{\frac{\left|L_{1}-L_{2}\right|}{\left|p_{1 n+\tau_{1}}\right|}-\frac{\left|u_{n+\tau_{1}}-w_{n+\tau_{1}}\right|}{\left|p_{1 n+\tau_{1}}\right|}\right.\right. \\
& -\frac{1}{\left|p_{1 n+\tau_{1}}\right|} \sum_{m=n+\tau_{1}}^{\infty} \mid f_{1}\left(m, u_{a_{1 m}}, \ldots, u_{a_{h m}}, v_{b_{1 m}}, \ldots, v_{b_{k m}}\right) \\
& \left.-f_{1}\left(m, w_{a_{1 m}}, \ldots, w_{a_{h m}}, z_{b_{1 m}}, \ldots, z_{b_{k m}}\right) \mid\right\} \\
& \sup _{n \geq \max \left\{T_{L_{1}}, T_{L_{2}}\right\}}\left\{\frac{\left|L_{1}-L_{2}\right|}{\left|p_{2 n+\tau_{2}}\right|}-\frac{\left|u_{n+\tau_{2}}-w_{n+\tau_{2}}\right|}{\left|p_{2 n+\tau_{2}}\right|}-\frac{1}{\left|p_{2 n+\tau_{2}}\right|}\right. \\
& \times \sum_{m=n+\tau_{2}}^{\infty} \mid f_{2}\left(m, u_{c_{1 m}}, \ldots, u_{c_{h m}}, v_{d_{1 m}}, \ldots, v_{d_{k m}}\right) \\
& \left.\left.-f_{2}\left(m, w_{c_{1 m}}, \ldots, w_{c_{h m}}, z_{d_{1 m}}, \ldots, z_{d_{k m}}\right) \mid\right\}\right\} \\
& \geq \max \left\{\frac{\left|L_{1}-L_{2}\right|}{\underline{p}}-\frac{\|u-w\|}{\bar{p}}\right. \\
& -\frac{1}{\bar{p}_{m=\max }} \sum_{\left.T_{L_{1}}, T_{L_{2}}\right\}}^{\infty} t_{1 m} \max \left\{\left|u_{a_{i m}}-w_{a_{i m}}\right|,\left|v_{b_{j m}}-z_{b_{j m}}\right|: i \in \Lambda_{h}, j \in \Lambda_{K}\right\}, \\
& \frac{\left|L_{1}-L_{2}\right|}{\underline{p}}-\frac{\|v-z\|}{\bar{p}} \\
& \left.-\frac{1}{\overline{\bar{p}}} \sum_{m=\max \left\{T_{L_{1}, T_{L_{2}}}\right\}}^{\infty} t_{2 m} \max \left\{\left|u_{c_{i m}}-w_{c_{i m}}\right|,\left|v_{d_{j m}}-z_{d_{j m}}\right|: i \in \Lambda_{h}, j \in \Lambda_{K}\right\}\right\} \\
& \geq \max \left\{\frac{\left|L_{1}-L_{2}\right|}{\underline{p}}-\frac{\|u-w\|}{\bar{p}}-\frac{1}{\bar{p}} \sum_{m=\max \left\{T_{L_{1}, T_{L_{2}}}\right\}}^{\infty} t_{1 m} \max \{\|u-w\|,\|v-z\|\},\right.
\end{aligned}
$$


Abstract and Applied Analysis

$$
\begin{gathered}
\frac{\left|L_{1}-L_{2}\right|}{\underline{p}}-\frac{\|v-z\|}{\bar{p}}-\frac{1}{\bar{p}} \sum_{m=\max \left\{T_{L_{1}}, T_{L_{2}}\right\}}^{\infty} t_{2 m} \max \{\|u-w\|,\|v-z\|\} \\
\frac{\left|L_{1}-L_{2}\right|}{\underline{p}}-\frac{1}{\bar{p}}\left(1+\max \left\{\theta_{L_{1}}, \theta_{L_{2}}\right\}\right)\|(u, v)-(w, z)\|_{1},
\end{gathered}
$$

which yields that

$$
\|(u, v)-(w, z)\|_{1} \geq \frac{\bar{p}\left|L_{1}-L_{2}\right|}{\underline{p}\left(\bar{p}+\max \left\{\theta_{L_{1}}, \theta_{L_{2}}\right\}\right)}>0,
$$

that is, $(u, v) \neq(w, z)$. This completes the proof.

Theorem 2.5. Assume that there exist constants $M, N, \bar{p}, p \in \mathbb{R}^{+} \backslash\{0\}, n_{1} \in \mathbb{N}_{n_{0}}$ and four nonnegative sequences $\left\{r_{1 n}\right\}_{n \in \mathbb{N}_{n_{0}}},\left\{r_{2 n}\right\}_{n \in \mathbb{N}_{n_{0}}},\left\{t_{1 n}\right\}_{n \in \mathbb{N}_{n_{0}}}$ and $\left\{t_{2 n}\right\}_{n \in \mathbb{N}_{n_{0}}}$ satisfying (2.1), (2.2), (2.24), and

$$
\frac{N\left(\bar{p}^{2}-\underline{p}\right)}{\bar{p}}<\frac{M\left(\underline{p}^{2}-\bar{p}\right)}{\underline{p}}, \quad 1<\underline{p} \leq p_{\text {in }} \leq \bar{p}<\underline{p}^{2}, \quad \forall n \geq n_{1}, \quad i \in \Lambda_{2} .
$$

Then,

(a) for each $L \in(\bar{p}(M / p+N), p(N / \bar{p}+M))$, there exist $\theta_{L} \in(0,1)$ and $T_{L} \geq \max \left\{\tau_{1}, \tau_{2}\right\}+$ $n_{1}+|\alpha|$ such that for any $\left(x^{0}, y^{0}\right)=\left(\left\{x_{n}^{0}\right\}_{n \in \mathbb{Z}_{\alpha}},\left\{y_{n}^{0}\right\}_{n \in \mathbb{Z}_{\alpha}}\right) \in A(N, M)$, the Mann iterative sequence with errors $\left\{\left(x^{\mu}, y^{\mu}\right)\right\}_{\mu \in \mathbb{N}_{0}}=\left\{\left(\left\{x_{n}^{\mu}\right\}_{n \in \mathbb{Z}_{\alpha}},\left\{y_{n}^{\mu}\right\}_{n \in \mathbb{Z}_{\alpha}}\right)\right\}_{\mu \in \mathbb{N}_{0}}$ generated by the schemes

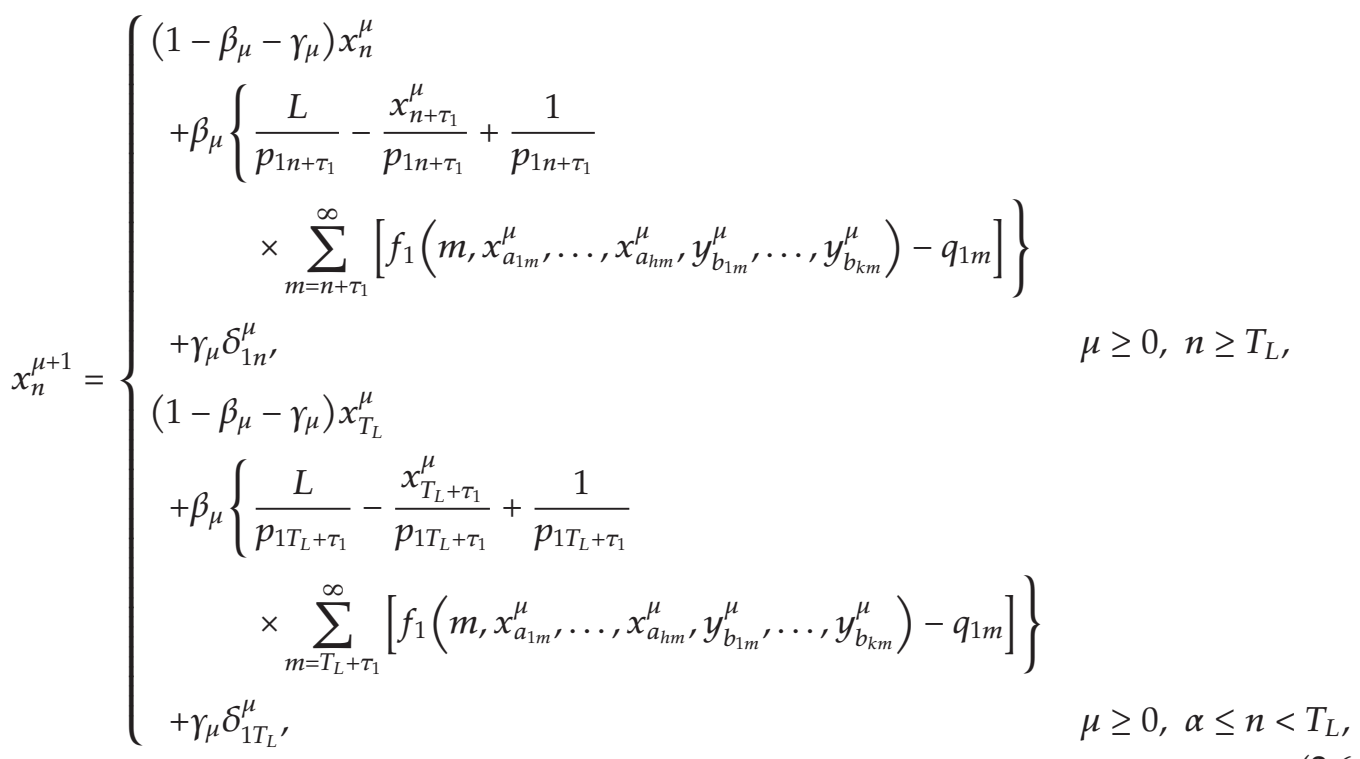




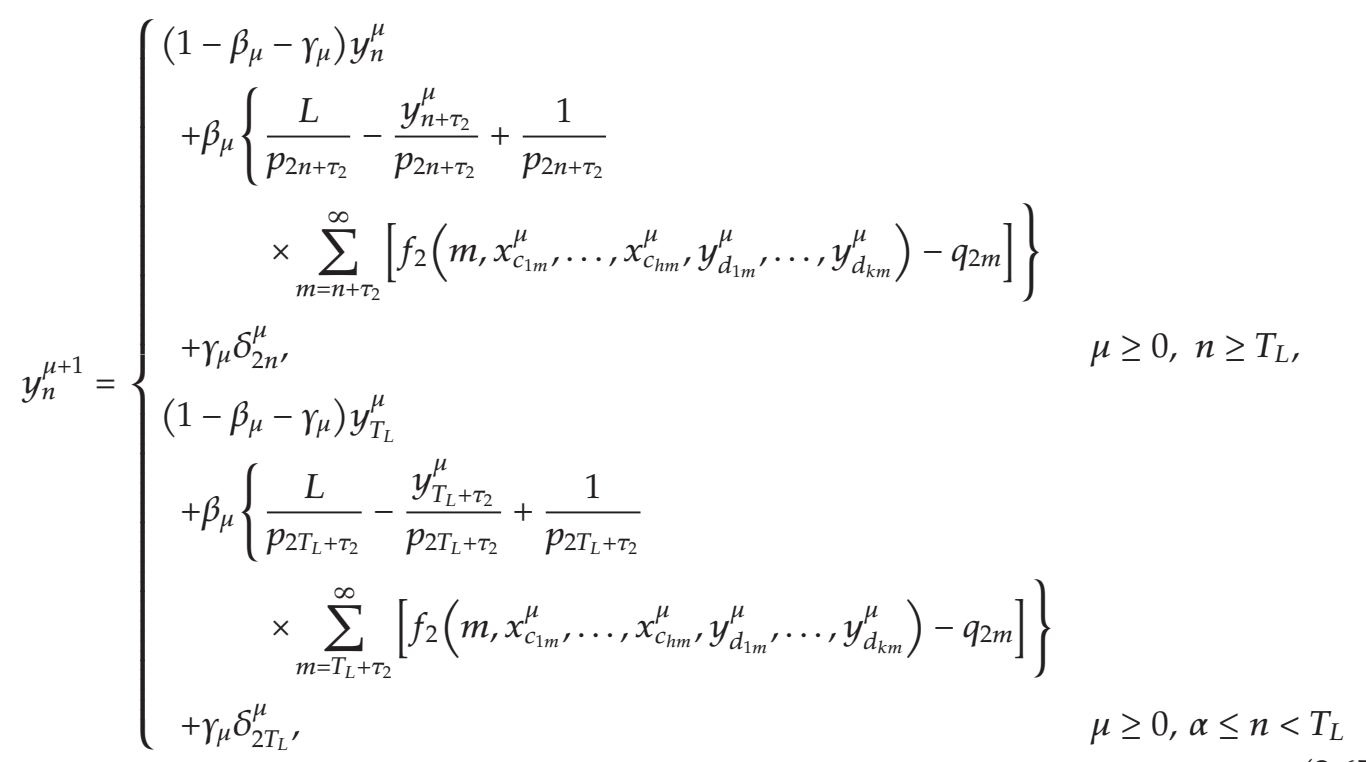

converges to a bounded positive solution $(x, y) \in A(N, M)$ of System (1.14) and has the error estimate (2.7), where $\left\{\beta_{\mu}\right\}_{\mu \in \mathbb{N}_{0}},\left\{\gamma_{\mu}\right\}_{\mu \in \mathbb{N}_{0}} \subset[0,1]$, and $\left\{\left(\delta_{1}^{\mu}, \delta_{2}^{\mu}\right)\right\}_{\mu \in \mathbb{N}_{0}}=\left\{\left(\left\{\delta_{1 n}^{\mu}\right\}_{n \in \mathbb{Z}_{\alpha^{\prime}}}\right.\right.$ $\left.\left.\left\{\delta_{2 n}^{\mu}\right\}_{n \in \mathbb{Z}_{\alpha}}\right)\right\}_{\mu \in \mathbb{N}_{0}} \subset A(N, M)$ are arbitrary sequences with (2.8) and (2.9),

(b) System (1.14) has uncountably many bounded positive solutions in $A(N, M)$.

Proof. (a) Let $L \in(\bar{p}(M / p+N), p(N / \bar{p}+M))$. Now we construct a contraction mapping $S_{L}: A(N, M) \rightarrow A(N, \bar{M})$ and prove that its fixed point is a bounded positive solution of System (1.14). Observe that (2.24) and (2.65) imply that there exist $\theta_{L} \in(0,1)$ and $T_{L} \geq$ $\max \left\{\tau_{1}, \tau_{2}\right\}+n_{1}+|\alpha|$ satisfying

$$
\begin{gathered}
\theta_{L}=\frac{1}{\underline{p}}\left(1+\sum_{m=T_{L}}^{\infty} \max \left\{t_{i m}: i \in \Lambda_{2}\right\}\right), \\
\sum_{m=T_{L}}^{\infty} \max \left\{r_{i m}+\left|q_{i m}\right|: i \in \Lambda_{2}\right\}<\min \left\{\frac{N \underline{p}}{\bar{p}}+M \underline{p}-L, \frac{L \underline{p}}{\bar{p}}-M-N \underline{p}\right\} .
\end{gathered}
$$

Define three mappings $S_{L}: A(N, M) \rightarrow l_{\alpha}^{\infty} \times l_{\alpha}^{\infty}, S_{1 L}, S_{2 L}: A(N, M) \rightarrow l_{\alpha}^{\infty}$ by (2.14),

$$
S_{1 L}\left(x_{n}, y_{n}\right)= \begin{cases}\frac{L}{p_{1 n+\tau_{1}}}-\frac{x_{n+\tau_{1}}}{p_{1 n+\tau_{1}}} & \\ +\frac{1}{p_{1 n+\tau_{1}}} \sum_{m=n+\tau_{1}}^{\infty}\left[f_{1}\left(m, x_{a_{1 m}}, \ldots, x_{a_{h m}}, y_{b_{1 m}}, \ldots, y_{b_{k m}}\right)-q_{1 m}\right], & n \geq T_{L} \\ S_{1 L}\left(x_{T_{L}}, y_{T_{L}}\right), & \alpha \leq n<T_{L}\end{cases}
$$




$$
S_{2 L}\left(x_{n}, y_{n}\right)= \begin{cases}\frac{L}{p_{2 n+\tau_{2}}}-\frac{y_{n+\tau_{2}}}{p_{2 n+\tau_{2}}} & \\ +\frac{1}{p_{2 n+\tau_{2}}} \sum_{m=n+\tau_{2}}^{\infty}\left[f_{2}\left(m, x_{c_{1 m}}, \ldots, x_{c_{h m}}, y_{d_{1 m}}, \ldots, y_{d_{k m}}\right)-q_{2 m}\right], & n \geq T_{L}, \\ S_{2 L}\left(x_{T_{L}}, y_{T_{L}}\right), & \alpha \leq n<T_{L},\end{cases}
$$

for all $(x, y)=\left(\left\{x_{n}\right\}_{n \in \mathbb{Z}_{\alpha}},\left\{y_{n}\right\}_{n \in \mathbb{Z}_{\alpha}}\right) \in A(N, M)$.

Using (2.1), (2.2), (2.14), (2.65), and (2.68)-(2.71), we deduce that for any $(x, y)=$ $\left(\left\{x_{n}\right\}_{n \in \mathbb{Z}_{\alpha}}\left\{y_{n}\right\}_{n \in \mathbb{Z}_{\alpha}}\right)$ and $(u, v)=\left(\left\{u_{n}\right\}_{n \in \mathbb{Z}_{\alpha}},\left\{v_{n}\right\}_{n \in \mathbb{Z}_{\alpha}}\right) \in A(N, M)$,

$$
\begin{aligned}
& \left\|S_{L}(x, y)-S_{L}(u, v)\right\|_{1} \\
& =\max \left\{\left\|S_{i L}(x, y)-S_{i L}(u, v)\right\|: i \in \Lambda_{2}\right\} \\
& =\max \left\{\sup _{n \geq T_{L}}\left|S_{i L}\left(x_{n}, y_{n}\right)-S_{i L}\left(u_{n}, v_{n}\right)\right|: i \in \Lambda_{2}\right\} \\
& \leq \max \left\{\operatorname { s u p } _ { n \geq T _ { L } } \left\{\frac { 1 } { | p _ { 1 n + \tau _ { 1 } } | } \left(\left|x_{n+\tau_{1}}-u_{n+\tau_{1}}\right|+\sum_{m=n+\tau_{1}}^{\infty} \mid f_{1}\left(m, x_{a_{1 m}}, \ldots, x_{a_{h m}}, y_{b_{1 m}}, \ldots, y_{b_{k m}}\right)\right.\right.\right. \\
& \left.\left.-f_{1}\left(m, u_{a_{1 m}}, \ldots, u_{a_{h m}}, v_{b_{1 m}}, \ldots, v_{b_{k m}}\right) \mid\right)\right\},
\end{aligned}
$$

$$
\begin{aligned}
\sup _{n \geq T_{L}}\left\{\frac { 1 } { | p _ { 2 n + \tau _ { 2 } } | } \left(\left|y_{n+\tau_{2}}-v_{n+\tau_{2}}\right|\right.\right. & \\
\quad+\sum_{m=n+\tau_{2}}^{\infty} \mid & f_{2}\left(m, x_{c_{1 m}}, \ldots, x_{c_{h m}}, y_{d_{1 m}}, \ldots, y_{d_{k m}}\right) \\
& \left.\left.\left.\quad-f_{2}\left(m, u_{c_{1 m}}, \ldots, u_{c_{h m}}, v_{d_{1 m}}, \ldots, v_{d_{k m}}\right) \mid\right)\right\}\right\}
\end{aligned}
$$$$
\leq \frac{1}{\underline{p}} \max \left\{\|x-u\|+\sum_{m=T_{L}}^{\infty} t_{1 m} \max \left\{\left|x_{a_{i m}}-u_{a_{i m}}\right|,\left|y_{b_{j m}}-v_{b_{j m}}\right|: i \in \Lambda_{h}, j \in \Lambda_{k}\right\},\right.
$$$$
\left.\|y-v\|+\sum_{m=T_{L}}^{\infty} t_{2 m} \max \left\{\left|x_{c_{i m}}-u_{c_{i m}}\right|,\left|y_{d_{j m}}-v_{d_{j m}}\right|: i \in \Lambda_{h}, j \in \Lambda_{k}\right\}\right\}
$$$$
\leq \frac{1}{\underline{p}} \max \left\{\|x-u\|+\sum_{m=T_{L}}^{\infty} t_{1 m} \max \{\|x-u\|,\|y-v\|\},\|y-v\|+\sum_{m=T_{L}}^{\infty} t_{2 m} \max \{\|x-u\|,\|y-v\|\}\right\}
$$$$
\leq \frac{1}{\underline{p}}\left(1+\sum_{m=T_{L}}^{\infty} \max \left\{t_{1 m}, t_{2 m}\right\}\right)\|(x, y)-(u, v)\|_{1}
$$

$=\theta_{L}\|(x, y)-(u, v)\|_{1}$ 


$$
\begin{aligned}
& S_{1 L}\left(x_{n}, y_{n}\right) \\
& =\frac{L}{p_{1 n+\tau_{1}}}-\frac{x_{n+\tau_{1}}}{p_{1 n+\tau_{1}}}+\frac{1}{p_{1 n+\tau_{1}}} \sum_{m=n+\tau_{1}}^{\infty}\left[f_{1}\left(m, x_{a_{1 m}}, \ldots, x_{a_{h m}}, y_{b_{1 m}}, \ldots, y_{b_{k m}}\right)-q_{1 m}\right] \\
& \leq \frac{L}{\underline{p}}-\frac{N}{\bar{p}}+\frac{1}{\underline{p}_{m}} \sum_{m=T_{L}+\tau_{1}}^{\infty}\left(r_{1 m}+\left|q_{1 m}\right|\right) \\
& \leq \frac{L}{\underline{p}}-\frac{N}{\bar{p}}+\frac{1}{p} \min \left\{\frac{N \underline{p}}{\bar{p}}+M \underline{p}-L, \frac{L \underline{p}}{\bar{p}}-M-N \underline{p}\right\} \\
& \leq M, \quad \forall n \geq T_{L}, \\
& S_{1 L}\left(x_{n}, y_{n}\right) \\
& =\frac{L}{p_{1 n+\tau_{1}}}-\frac{x_{n+\tau_{1}}}{p_{1 n+\tau_{1}}}+\frac{1}{p_{1 n+\tau_{1}}} \sum_{m=n+\tau_{1}}^{\infty}\left[f_{1}\left(m, x_{a_{1 m}}, \ldots, x_{a_{h m}}, y_{b_{1 m}}, \ldots, y_{b_{k m}}\right)-q_{1 m}\right] \\
& \geq \frac{L}{\bar{p}}-\frac{M}{\underline{p}}-\frac{1}{\underline{p}_{m=T_{L}+\tau_{1}}} \sum_{1 m}^{\infty}\left(r_{1 m}+\left|q_{1 m}\right|\right) \\
& \geq \frac{L}{\bar{p}}-\frac{M}{\underline{p}}-\frac{1}{p} \min \left\{\frac{N \underline{p}}{\bar{p}}+M \underline{p}-L, \frac{L}{\bar{p}}-M-N \underline{p}\right\} \\
& \geq N, \quad \forall n \geq T_{L}, \\
& N \leq S_{2 L}\left(x_{n}, y_{n}\right) \leq M, \quad \forall n \geq T_{L},
\end{aligned}
$$

which yield (2.16) and (2.17), and which guarantee that $S_{L}$ is a self-mapping from $A(N, M)$ into itself and is a contraction by $\theta_{L} \in(0,1)$. Thus the Banach fixed point theorem means that $S_{L}$ has a unique fixed point $(x, y)=\left(\left\{x_{n}\right\}_{n \in \mathbb{Z}_{\alpha}},\left\{y_{n}\right\}_{n \in \mathbb{Z}_{\alpha}}\right) \in A(N, M)$, that is,

$$
\begin{aligned}
& x_{n}=\frac{L}{p_{1 n+\tau_{1}}}-\frac{x_{n+\tau_{1}}}{p_{1 n+\tau_{1}}}+\frac{1}{p_{1 n+\tau_{1}}} \sum_{m=n+\tau_{1}}^{\infty}\left[f_{1}\left(m, x_{a_{1 m}}, \ldots, x_{a_{h m}}, y_{b_{1 m}}, \ldots, y_{b_{k m}}\right)-q_{1 m}\right], \quad n \geq T_{L}, \\
& y_{n}=\frac{L}{p_{2 n+\tau_{2}}}-\frac{y_{n+\tau_{2}}}{p_{2 n+\tau_{2}}}+\frac{1}{p_{2 n+\tau_{2}}} \sum_{m=n+\tau_{2}}^{\infty}\left[f_{2}\left(m, x_{c_{1 m}}, \ldots, x_{c_{h m}}, y_{d_{1 m}}, \ldots, y_{d_{k m}}\right)-q_{2 m}\right], \quad n \geq T_{L},
\end{aligned}
$$


which yield that

$$
\begin{gathered}
x_{n}+p_{1 n} x_{n-\tau_{1}}=L+\sum_{m=n}^{\infty}\left[f_{1}\left(m, x_{a_{1 m}}, \ldots, x_{a_{h m}}, y_{b_{1 m}}, \ldots, y_{b_{k m}}\right)-q_{1 m}\right], \quad n \geq T_{L}+\tau_{1}, \\
y_{n}+p_{2 n} y_{n-\tau_{2}}=L+\sum_{m=n}^{\infty}\left[f_{2}\left(m, x_{c_{1 m}}, \ldots, x_{c_{h m}}, y_{d_{1 m}}, \ldots, y_{d_{k m}}\right)-q_{2 m}\right], \quad n \geq T_{L}+\tau_{2}, \\
\Delta\left(x_{n}+p_{1 n} x_{n-\tau_{1}}\right)=-f_{1}\left(n, x_{a_{1 n}}, \ldots, x_{a_{h n}}, y_{b_{1 n}}, \ldots, y_{b_{k n}}\right)+q_{1 n}, \quad \forall n \geq T_{L}+\tau_{1}, \\
\Delta\left(y_{n}+p_{2 n} y_{n-\tau_{2}}\right)=-f_{2}\left(n, x_{c_{1 n}}, \ldots, x_{c_{h n}}, y_{d_{1 n}}, \ldots, y_{d_{k n}}\right)+q_{2 n}, \quad \forall n \geq T_{L}+\tau_{2},
\end{gathered}
$$

that is, $(x, y)$ is a bounded positive solution of the system (1.14) in $A(N, M)$.

In view of (2.14), (2.16), (2.65)-(2.67), (2.70), and (2.71), we gain that

$$
\begin{aligned}
& \left\|\left(x^{\mu+1}, y^{\mu+1}\right)-(x, y)\right\|_{1} \\
& =\max \left\{\sup _{n \geq T_{L}}\left|x_{n}^{\mu+1}-x_{n}\right|, \sup _{n \geq T_{L}}\left|y_{n}^{\mu+1}-y_{n}\right|\right\} \\
& \leq \max \left\{\operatorname { s u p } _ { n \geq T _ { L } } \left[\left(1-\beta_{\mu}-\gamma_{\mu}\right)\left|x_{n}^{\mu}-x_{n}\right|+\beta_{\mu}\right.\right. \\
& \times\left|\frac{L}{p_{1 n+\tau_{1}}}-\frac{x_{n+\tau_{1}}^{\mu}}{p_{1 n+\tau_{1}}}+\frac{1}{p_{1 n+\tau_{1}}} \sum_{m=n}^{\infty}\left[f_{1}\left(m, x_{a_{1 m}}^{\mu}, \ldots, x_{a_{h m}}^{\mu}, y_{b_{1 m}}^{\mu}, \ldots, y_{b_{k m}}^{\mu}\right)-q_{1 m}\right]-x_{n}\right| \\
& \left.+\gamma_{\mu}\left|\delta_{1 n}^{\mu}-x_{n}\right|\right] \\
& \sup _{n \geq T_{L}}\left[\left(1-\beta_{\mu}-\gamma_{\mu}\right)\left|y_{n}^{\mu}-y_{n}\right|+\beta_{\mu}\right. \\
& \times\left|\frac{L}{p_{2 n+\tau_{2}}}-\frac{y_{n+\tau_{2}}^{\mu}}{p_{2 n+\tau_{2}}}+\frac{1}{p_{2 n+\tau_{2}}} \sum_{m=n}^{\infty}\left[f_{2}\left(m, x_{c_{1 m}}^{\mu}, \ldots, x_{c_{h m}}^{\mu}, y_{d_{1 m}}^{\mu}, \ldots, y_{d_{k m}}^{\mu}\right)-q_{2 m}\right]-y_{n}\right| \\
& \left.\left.+\gamma_{\mu}\left|\delta_{2 n}^{\mu}-y_{n}\right|\right]\right\}
\end{aligned}
$$




$$
\begin{aligned}
& \leq \max \left\{\sup _{n \geq T_{L}}\left[\left(1-\beta_{\mu}-\gamma_{\mu}\right)\left\|x^{\mu}-x\right\|+\beta_{\mu}\left|S_{1 L}\left(x_{n}^{\mu}, y_{n}^{\mu}\right)-S_{1 L}\left(x_{n}, y_{n}\right)\right|+2 M \gamma_{\mu}\right],\right. \\
& \left.\sup _{n \geq T_{L}}\left[\left(1-\beta_{\mu}-\gamma_{\mu}\right)\left\|y^{\mu}-y\right\|+\beta_{\mu}\left|S_{2 L}\left(x_{n}^{\mu}, y_{n}^{\mu}\right)-S_{2 L}\left(x_{n}, y_{n}\right)\right|+2 M \gamma_{\mu}\right]\right\} \\
& \leq\left(1-\beta_{\mu}\left(1-\theta_{L}\right)\right)\left\|\left(x^{\mu}, y^{\mu}\right)-(x, y)\right\|_{1}+2 M \gamma_{\mu}, \quad \forall \mu \geq 0,
\end{aligned}
$$

which implies (2.7). Thus, Lemma 1.1 and (2.7)-(2.9) imply that $\lim _{\mu \rightarrow \infty}\left\|\left(x^{\mu}, y^{\mu}\right)-(x, y)\right\|_{1}=$ 0 .

(b) Let $L_{1}, L_{2} \in(N, M)$ and $L_{1} \neq L_{2}$. Similar to the proof of (a), we conclude that for each $i \in \Lambda_{2}$, there exist a constant $\theta_{L_{i}} \in(0,1)$, a positive integer $T_{L_{i}} \geq \max \left\{\tau_{1}, \tau_{2}\right\}+n_{1}+|\alpha|$, and mappings $S_{L_{i}}, S_{1 L_{i}}, S_{2 L_{i}}$ satisfying (2.14) and (2.68)-(2.71), where $L, \theta_{L}$, and $T_{L}$ are replaced by $L_{i}, \theta_{L_{i}}$, and $T_{L_{i}}$, respectively, and the contraction mappings $S_{L_{1}}$ and $S_{L_{2}}$ have the unique fixed points $(u, v)=\left(\left\{u_{n}\right\}_{n \in \mathbb{Z}_{\alpha}},\left\{v_{n}\right\}_{n \in \mathbb{Z}_{\alpha}}\right),(w, z)=\left(\left\{x_{n}\right\}_{n \in \mathbb{Z}_{\alpha}},\left\{y_{n}\right\}_{n \in \mathbb{Z}_{\alpha}}\right) \in A(N, M)$, which are bounded positive solutions of System (1.14) in $A(N, M)$, respectively. In order to show that System (1.14) possesses uncountably many bounded positive solutions in $A(N, M)$, we prove only that $(u, v) \neq(w, z)$. In light of (2.14) and (2.68)-(2.71), we have

$$
\begin{aligned}
& \|(u, v)-(w, z)\|_{1} \\
& =\max \{\|u-w\|,\|v-z\|\} \\
& \geq \max \left\{\sup _{n \geq \max \left\{T_{L_{1}}, T_{L_{2}}\right\}}\left|u_{n}-w_{n}\right|, \sup _{n \geq \max \left\{T_{L_{1}}, T_{L_{2}}\right\}}\left|v_{n}-z_{n}\right|\right\} \\
& \geq \max \left\{\operatorname { s u p } _ { n \geq \operatorname { m a x } \{ T _ { L _ { 1 } } , T _ { L _ { 2 } } \} } \left\{\frac{\left|L_{1}-L_{2}\right|}{\left|p_{1 n+\tau_{1}}\right|}-\frac{\left|u_{n+\tau_{1}}-w_{n+\tau_{1}}\right|}{\left|p_{1 n+\tau_{1}}\right|}\right.\right. \\
& -\frac{1}{\left|p_{1 n+\tau_{1}}\right|} \sum_{m=n+\tau_{1}}^{\infty} \mid f_{1}\left(m, u_{a_{1 m}}, \ldots, u_{a_{h m}}, v_{b_{1 m}}, \ldots, v_{b_{k m}}\right) \\
& \left.-f_{1}\left(m, w_{a_{1 m}}, \ldots, w_{a_{h m}}, z_{b_{1 m}}, \ldots, z_{b_{k m}}\right) \mid\right\} \\
& \sup _{n \geq \max \left\{T_{L_{1}}, T_{L_{2}}\right\}}\left\{\frac{\left|L_{1}-L_{2}\right|}{\left|p_{2 n+\tau_{2}}\right|}-\frac{\left|u_{n+\tau_{2}}-w_{n+\tau_{2}}\right|}{\left|p_{2 n+\tau_{2}}\right|}-\frac{1}{\left|p_{2 n+\tau_{2}}\right|}\right. \\
& \times \sum_{m=n+\tau_{2}}^{\infty} \mid f_{2}\left(m, u_{c_{1 m}}, \ldots, u_{c_{h m}}, v_{d_{1 m}}, \ldots, v_{d_{k m}}\right) \\
& \left.\left.-f_{2}\left(m, w_{c_{1 m}}, \ldots, w_{c_{h m}}, z_{d_{1 m}}, \ldots, z_{d_{k m}}\right) \mid\right\}\right\}
\end{aligned}
$$


Abstract and Applied Analysis

$$
\begin{aligned}
& \geq \max \left\{\frac{\left|L_{1}-L_{2}\right|}{\bar{p}}-\frac{\|u-w\|}{\underline{p}}\right. \\
& -\frac{1}{\underline{p}_{m}} \sum_{m=\max \left\{T_{L_{1}}, T_{L_{2}}\right\}}^{\infty} t_{1 m} \max \left\{\left|u_{a_{l m}}-w_{a_{l m}}\right|,\left|v_{b_{j m}}-z_{b_{j m}}\right|: l \in \Lambda_{h}, j \in \Lambda_{K}\right\}, \\
& \frac{\left|L_{1}-L_{2}\right|}{\bar{p}}-\frac{\|v-z\|}{\underline{p}} \\
& \left.-\frac{1}{\underline{p}_{m}} \sum_{m=\max \left\{T_{L_{1}, T_{L_{2}}}\right\}}^{\infty} t_{2 m} \max \left\{\left|u_{c_{l m}}-w_{c_{l m}}\right|,\left|v_{d_{j m}}-z_{d_{j m}}\right|: l \in \Lambda_{h}, j \in \Lambda_{K}\right\}\right\} \\
& \geq \max \left\{\frac{\left|L_{1}-L_{2}\right|}{\bar{p}}-\frac{\|u-w\|}{\underline{p}}-\frac{1}{p} \sum_{m=\max \left\{T_{L_{1}}, T_{L_{2}}\right\}}^{\infty} t_{1 m} \max \{\|u-w\|,\|v-z\|\},\right. \\
& \left.\frac{\left|L_{1}-L_{2}\right|}{\bar{p}}-\frac{\|v-z\|}{\underline{p}}-\frac{1}{\underline{p}} \sum_{m=\max \left\{T_{L_{1}, T_{L_{2}}}\right\}}^{\infty} t_{2 m} \max \{\|u-w\|,\|v-z\|\}\right\} \\
& \geq \frac{\left|L_{1}-L_{2}\right|}{\bar{p}}-\frac{1}{\underline{p}}\left(1+\max \left\{\theta_{L_{1}}, \theta_{L_{2}}\right\}\right)\|(u, v)-(w, z)\|_{1},
\end{aligned}
$$

which yields that

$$
\|(u, v)-(w, z)\|_{1} \geq \frac{\underline{p}\left|L_{1}-L_{2}\right|}{\bar{p}\left(\underline{p}+\max \left\{\theta_{L_{1}}, \theta_{L_{2}}\right\}\right)}>0,
$$

that is, $(u, v) \neq(w, z)$. This completes the proof.

Remark 2.6. Let $\left(a_{1}, b_{1}\right)$ and $\left(a_{2}, b_{2}\right)$ be two arbitrary intervals in $\mathbb{R}$. It is easy to see that

$$
\left(a_{1}, b_{1}\right) \cap\left(a_{2}, b_{2}\right) \neq \emptyset \Longleftrightarrow \max \left\{a_{1}, a_{2}\right\}<\min \left\{b_{1}, b_{2}\right\}
$$

From Remark 2.6 and Theorems 2.1-2.5, we can obtain the following. 
Theorem 2.7. Assume that there exist constants $M, N \in \mathbb{R}^{+} \backslash\{0\}, n_{1} \in \mathbb{N}_{n_{0}}$ and four nonnegative sequences $\left\{r_{1 n}\right\}_{n \in \mathbb{N}_{n_{0}}},\left\{r_{2 n}\right\}_{n \in \mathbb{N}_{n_{0}}},\left\{t_{1 n}\right\}_{n \in \mathbb{N}_{n_{0}}}$, and $\left\{t_{2 n}\right\}_{n \in \mathbb{N}_{n_{0}}}$ satisfying (2.1), (2.2),

$$
\begin{gathered}
\sum_{n=n_{0}}^{\infty} n \max \left\{r_{1 n}, t_{1 n},\left|q_{1 n}\right|\right\}<+\infty, \\
\sum_{n=n_{0}}^{\infty} \max \left\{r_{2 n}, t_{2 n},\left|q_{2 n}\right|\right\}<+\infty, \\
N<M, \quad p_{1 n}=-1, \quad \forall n \geq n_{1}, \\
p_{2 n}=1, \quad \forall n \geq n_{1} .
\end{gathered}
$$

Then,

(a) for each $L \in(N, M)$, there exist $\theta_{L} \in(0,1)$ and $T_{L} \geq \max \left\{\tau_{1}, \tau_{2}\right\}+n_{1}+|\alpha|$ such that for any $\left(x^{0}, y^{0}\right)=\left(\left\{x_{n}^{0}\right\}_{n \in \mathbb{Z}_{\alpha}},\left\{y_{n}^{0}\right\}_{n \in \mathbb{Z}_{\alpha}}\right) \in A(N, M)$, the Mann iterative sequence with errors $\left\{\left(x^{\mu}, y^{\mu}\right)\right\}_{\mu \in \mathbb{N}_{0}}=\left\{\left(\left\{x_{n}^{\mu}\right\}_{n \in \mathbb{Z}_{\alpha}},\left\{y_{n}^{\mu}\right\}_{n \in \mathbb{Z}_{\alpha}}\right)\right\}_{\mu \in \mathbb{N}_{0}}$ generated by Schemes (2.5) and (2.27) converges to a bounded positive solution $(x, y) \in A(N, M)$ of System (1.14) and has the error estimate (2.7), where $\left\{\beta_{\mu}\right\}_{\mu \in \mathbb{N}_{0}},\left\{\gamma_{\mu}\right\}_{\mu \in \mathbb{N}_{0}} \subset[0,1]$ and $\left\{\left(\delta_{1}^{\mu}, \delta_{2}^{\mu}\right)\right\}_{\mu \in \mathbb{N}_{0}}=$ $\left\{\left(\left\{\delta_{1 n}^{\mu}\right\}_{n \in \mathbb{Z}_{\alpha}}\left\{\delta_{2 n}^{\mu}\right\}_{n \in \mathbb{Z}_{\alpha}}\right)\right\}_{\mu \in \mathbb{N}_{0}} \subset A(N, M)$ are arbitrary sequences with (2.8) and (2.9),

(b) System (1.14) has uncountably many bounded positive solutions in $A(N, M)$.

Theorem 2.8. Assume that there exist constants $M, N \in \mathbb{R}^{+} \backslash\{0\}, \bar{p}, p \in \mathbb{R}^{+}, n_{1} \in \mathbb{N}_{n_{0}}$, and four nonnegative sequences $\left\{r_{1 n}\right\}_{n \in \mathbb{N}_{n_{0}}},\left\{r_{2 n}\right\}_{n \in \mathbb{N}_{n_{0}}},\left\{t_{1 n}\right\}_{n \in \mathbb{N}_{n_{0}}}$ and $\left\{t_{2 n}\right\}_{n \in \mathbb{N}_{n_{0}}}$ satisfying (2.1), (2.2), (2.79)-(2.81),

$$
N<(1-\underline{p}-\bar{p}) M, \quad \underline{p}+\bar{p}<1, \quad-\underline{p} \leq p_{2 n} \leq \bar{p}, \quad \forall n \geq n_{1} .
$$

Then,

(a) for each $L \in(N, M) \cap(N+\bar{p} M, M(1-p))$, there exist $\theta_{L} \in(0,1)$ and $T_{L} \geq \max \left\{\tau_{1}, \tau_{2}\right\}+$ $n_{1}+|\alpha|$ such that for any $\left(x^{0}, y^{0}\right)=\left(\left\{x_{n}^{0}\right\}_{n \in \mathbb{Z}_{\alpha}},\left\{y_{n}^{0}\right\}_{n \in \mathbb{Z}_{\alpha}}\right) \in A(N, M)$, the Mann iterative sequence with errors $\left\{\left(x^{\mu}, y^{\mu}\right)\right\}_{\mu \in \mathbb{N}_{0}}=\left\{\left(\left\{x_{n}^{\mu}\right\}_{n \in \mathbb{Z}_{\alpha}},\left\{y_{n}^{\mu}\right\}_{n \in \mathbb{Z}_{\alpha}}\right)\right\}_{\mu \in \mathbb{N}_{0}}$ generated by Schemes (2.5) and (2.41) converges to a bounded positive solution $(x, y) \in A(N, M)$ of System (1.14) and has the error estimate (2.7), where $\left\{\beta_{\mu}\right\}_{\mu \in \mathbb{N}_{0}},\left\{\gamma_{\mu}\right\}_{\mu \in \mathbb{N}_{0}} \subset[0,1]$ and $\left\{\left(\delta_{1}^{\mu}, \delta_{2}^{\mu}\right)\right\}_{\mu \in \mathbb{N}_{0}}=\left\{\left(\left\{\delta_{1 n}^{\mu}\right\}_{n \in \mathbb{Z}_{\alpha^{\prime}}},\left\{\delta_{2 n}^{\mu}\right\}_{n \in \mathbb{Z}_{\alpha}}\right)\right\}_{\mu \in \mathbb{N}_{0}} \subset A(N, M)$ are arbitrary sequences with (2.8) and (2.9),

(b) System (1.14) has uncountably many bounded positive solutions in $A(N, M)$.

Theorem 2.9. Assume that there exist constants $M, N, \bar{p}, p \in \mathbb{R}^{+} \backslash\{0\}, n_{1} \in \mathbb{N}_{n_{0}}$ and four nonnegative sequences $\left\{r_{1 n}\right\}_{n \in \mathbb{N}_{n_{0}}},\left\{r_{2 n}\right\}_{n \in \mathbb{N}_{n_{0}}},\left\{t_{1 n}\right\}_{n \in \mathbb{N}_{n_{0}}}$ and $\left\{t_{2 n}\right\}_{n \in \mathbb{N}_{n_{0}}}$ satisfying (2.1), (2.2), (2.79)-(2.81),

$$
\begin{gathered}
0<N(\underline{p}-1)<M(\bar{p}-1), \quad-\underline{p} \leq p_{2 n} \leq-\bar{p}, \quad \forall n \geq n_{1}, \\
\max \{N, N(\underline{p}-1)\}<\min \{M, M(\bar{p}-1)\} .
\end{gathered}
$$


Then,

(a) for each $L \in(N, M) \cap(N(p-1), M(\bar{p}-1))$, there exist $\theta_{L} \in(0,1)$ and $T_{L} \geq \max \left\{\tau_{1}, \tau_{2}\right\}+$ $n_{1}+|\alpha|$ such that for any $\left(x^{0}, y^{0}\right)=\left(\left\{x_{n}^{0}\right\}_{n \in \mathbb{Z}_{\alpha}},\left\{y_{n}^{0}\right\}_{n \in \mathbb{Z}_{\alpha}}\right) \in A(N, M)$, the Mann iterative sequence with errors $\left\{\left(x^{\mu}, y^{\mu}\right)\right\}_{\mu \in \mathbb{N}_{0}}=\left\{\left(\left\{x_{n}^{\mu}\right\}_{n \in \mathbb{Z}_{\alpha}},\left\{y_{n}^{\mu}\right\}_{n \in \mathbb{Z}_{\alpha}}\right)\right\}_{\mu \in \mathbb{N}_{0}}$ generated by Schemes (2.5) and (2.54) converges to a bounded positive solution $(x, y) \in A(N, M)$ of System (1.14) and has the error estimate (2.7), where $\left\{\beta_{\mu}\right\}_{\mu \in \mathbb{N}_{0}},\left\{\gamma_{\mu}\right\}_{\mu \in \mathbb{N}_{0}} \subset[0,1]$ and $\left.\left\{\left(\delta_{1}^{\mu}, \delta_{2}^{\mu}\right)\right\}_{\mu \in \mathbb{N}_{0}}=\left\{\left(\left\{\delta_{1 n}^{\mu}\right\}_{n \in \mathbb{Z}_{\alpha^{\prime}}}, \delta_{2 n}^{\mu}\right\}_{n \in \mathbb{Z}_{\alpha}}\right)\right\}_{\mu \in \mathbb{N}_{0}} \subset A(N, M)$ are arbitrary sequences with (2.8) and (2.9),

(b) System (1.14) has uncountably many bounded positive solutions in $A(N, M)$.

Theorem 2.10. Assume that there exist constants $M, N, M_{0}, N_{0}, \bar{p}_{0}, p \in \mathbb{R}^{+} \backslash\{0\}, n_{1} \in \mathbb{N}_{n_{0}}$ and four nonnegative sequences $\left\{r_{1 n}\right\}_{n \in \mathbb{N}_{n_{0}}},\left\{r_{2 n}\right\}_{n \in \mathbb{N}_{n_{0}}},\left\{t_{1 n}\right\}_{n \in \mathbb{N}_{n_{0}}}$ and $\left\{t_{2 n}\right\}_{n \in \mathbb{N}_{n_{0}}}$ satisfying (2.1), (2.2) with $i=1,(2.79)-(2.81)$,

$$
\begin{gathered}
\left|f_{2}\left(n, u_{1}, \ldots, u_{h}, v_{1}, \ldots, v_{k}\right)\right| \leq r_{2 n}, \quad \forall\left(n, u_{l}, v_{j}\right) \in \mathbb{N}_{n_{1}} \times\left[N_{0}, M_{0}\right]^{2}, l \in \Lambda_{h}, j \in \Lambda_{k}, \\
\left|f_{2}\left(n, u_{1}, \ldots, u_{h}, v_{1}, \ldots, v_{k}\right)-f_{2}\left(n, w_{1}, \ldots, w_{h}, z_{1}, \ldots, z_{k}\right)\right| \\
\leq t_{2 n} \max \left\{\left|u_{l}-w_{l}\right|,\left|v_{j}-z_{j}\right|: l \in \Lambda_{h}, j \in \Lambda_{k}\right\} \\
\forall\left(n, u_{l}, w_{l}, v_{j}, z_{j}\right) \in \mathbb{N}_{n_{1}} \times\left[N_{0}, M_{0}\right]^{4}, l \in \Lambda_{h}, j \in \Lambda_{k}, \\
\frac{N_{0}\left(\bar{p}_{0}^{2}-\underline{p}_{0}\right)}{\bar{p}_{0}}<\frac{M_{0}\left(\underline{p}_{0}^{2}-\bar{p}_{0}\right)}{\underline{p}_{0}}, \quad 1<\underline{p}_{0} \leq p_{2 n} \leq \bar{p}_{0}<\underline{p}_{0^{\prime}}^{2} \quad \forall n \geq n_{1} \\
\max \left\{N, \bar{p}_{0}\left(\frac{M_{0}}{\underline{p}_{0}}+N_{0}\right)\right\}<\min \left\{M, \underline{p}_{0}\left(\frac{N_{0}}{\bar{p}_{0}}+M_{0}\right)\right\}
\end{gathered}
$$

Then,

(a) for each $L \in(N, M) \cap\left(\bar{p}_{0}\left(M_{0} / \underline{p}_{0}+N_{0}\right), \underline{p}_{0}\left(N_{0} / \bar{p}_{0}+M_{0}\right)\right)$, there exist $\theta_{L} \in$ $(0,1)$ and $T_{L} \geq \max \left\{\tau_{1}, \tau_{2}\right\}+n_{1}+|\alpha|$ such that for any $\left(x^{0}, y^{0}\right)=\left(\left\{x_{n}^{0}\right\}_{n \in \mathbb{Z}_{\alpha^{\prime}}}\right.$ $\left.\left\{y_{n}^{0}\right\}_{n \in \mathbb{Z}_{\alpha}}\right) \in A\left(N, M, N_{0}, M_{0}\right)$, the Mann iterative sequence with errors $\left\{\left(x^{\mu}, y^{\mu}\right)\right\}_{\mu \in \mathbb{N}_{0}}=$ $\left\{\left(\left\{x_{n}^{\mu}\right\}_{n \in \mathbb{Z}_{\alpha}}\left\{y_{n}^{\mu}\right\}_{n \in \mathbb{Z}_{\alpha}}\right)\right\}_{\mu \in \mathbb{N}_{0}}$ generated by Schemes (2.5) and (2.67) converges to a bounded positive solution $(x, y) \in A\left(N, M, N_{0}, M_{0}\right)$ of System (1.14) and has the error estimate (2.7), where $\left\{\beta_{\mu}\right\}_{\mu \in \mathbb{N}_{0}},\left\{\gamma_{\mu}\right\}_{\mu \in \mathbb{N}_{0}} \subset[0,1]$, and $\left\{\left(\delta_{1}^{\mu}, \delta_{2}^{\mu}\right)\right\}_{\mu \in \mathbb{N}_{0}}=\left\{\left(\left\{\delta_{1 n}^{\mu}\right\}_{n \in \mathbb{Z}_{\alpha^{\prime}}}\right.\right.$ $\left.\left.\left\{\delta_{2 n}^{\mu}\right\}_{n \in \mathbb{Z}_{\alpha}}\right)\right\}_{\mu \in \mathbb{N}_{0}} \subset A\left(N, M, N_{0}, M_{0}\right)$ are arbitrary sequences with (2.8) and (2.9),

(b) the system (1.14) has uncountably many bounded positive solutions in $A\left(N, M, N_{0}, M_{0}\right)$.

Theorem 2.11. Assume that there exist constants $M, N \in \mathbb{R}^{+} \backslash\{0\}, \bar{p}, p \in \mathbb{R}^{+}, n_{1} \in \mathbb{N}_{n_{0}}$ and four nonnegative sequences $\left\{r_{1 n}\right\}_{n \in \mathbb{N}_{n_{0}}},\left\{r_{2 n}\right\}_{n \in \mathbb{N}_{n_{0}}},\left\{t_{1 n}\right\}_{n \in \mathbb{N}_{n_{0}}}$ and $\left\{t_{2 n}\right\}_{n \in \mathbb{N}_{n_{0}}}$ satisfying (2.1), (2.2), (2.24), (2.83) and

$$
N<M, \quad p_{1 n}=1, \quad \forall n \geq n_{1} .
$$


Then,

(a) for each $L \in(N, M) \cap(N+\bar{p} M, M(1-p))$, there exist $\theta_{L} \in(0,1)$ and $T_{L} \geq \max \left\{\tau_{1}, \tau_{2}\right\}+$ $n_{1}+|\alpha|$ such that for any $\left(x^{0}, y^{0}\right)=\left(\left\{x_{n}^{0}\right\}_{n \in \mathbb{Z}_{\alpha}},\left\{y_{n}^{0}\right\}_{n \in \mathbb{Z}_{\alpha}}\right) \in A(N, M)$, the Mann iterative sequence with errors $\left\{\left(x^{\mu}, y^{\mu}\right)\right\}_{\mu \in \mathbb{N}_{0}}=\left\{\left(\left\{x_{n}^{\mu}\right\}_{n \in \mathbb{Z}_{\alpha}},\left\{y_{n}^{\mu}\right\}_{n \in \mathbb{Z}_{\alpha}}\right)\right\}_{\mu \in \mathbb{N}_{0}}$ generated by Schemes (2.26) and (2.41) converges to a bounded positive solution $(x, y) \in A(N, M)$ of System (1.14) and has the error estimate (2.7), where $\left\{\beta_{\mu}\right\}_{\mu \in \mathbb{N}_{0}},\left\{\gamma_{\mu}\right\}_{\mu \in \mathbb{N}_{0}} \subset[0,1]$, and $\left\{\left(\delta_{1}^{\mu}, \delta_{2}^{\mu}\right)\right\}_{\mu \in \mathbb{N}_{0}}=\left\{\left(\left\{\delta_{1 n}^{\mu}\right\}_{n \in \mathbb{Z}_{\alpha}},\left\{\delta_{2 n}^{\mu}\right\}_{n \in \mathbb{Z}_{\alpha}}\right)\right\}_{\mu \in \mathbb{N}_{0}} \subset A(N, M)$ are arbitrary sequences with (2.8) and (2.9),

(b) System (1.14) has uncountably many bounded positive solutions in $A(N, M)$.

Theorem 2.12. Assume that there exist constants $M, N, \bar{p}, p \in \mathbb{R}^{+} \backslash\{0\}, n_{1} \in \mathbb{N}_{n_{0}}$ and four nonnegative sequences $\left\{r_{1 n}\right\}_{n \in \mathbb{N}_{n_{0}}},\left\{r_{2 n}\right\}_{n \in \mathbb{N}_{n_{0}}},\left\{t_{1 n}\right\}_{n \in \mathbb{N}_{n_{0}}}$ and $\left\{t_{2 n}\right\}_{n \in \mathbb{N}_{n_{0}}}$ satisfying (2.1), (2.2), (2.24), (2.84), (2.85), and (2.90). Then,

(a) for each $L \in(N, M) \cap(N(p-1), M(\bar{p}-1))$, there exist $\theta_{L} \in(0,1)$ and $T_{L} \geq \max \left\{\tau_{1}, \tau_{2}\right\}+$ $n_{1}+|\alpha|$ such that for any $\left(x^{0}, y^{0}\right)=\left(\left\{x_{n}^{0}\right\}_{n \in \mathbb{Z}_{\alpha}},\left\{y_{n}^{0}\right\}_{n \in \mathbb{Z}_{\alpha}}\right) \in A(N, M)$, the Mann iterative sequence with errors $\left\{\left(x^{\mu}, y^{\mu}\right)\right\}_{\mu \in \mathbb{N}_{0}}=\left\{\left(\left\{x_{n}^{\mu}\right\}_{n \in \mathbb{Z}_{\alpha}},\left\{y_{n}^{\mu}\right\}_{n \in \mathbb{Z}_{\alpha}}\right)\right\}_{\mu \in \mathbb{N}_{0}}$ generated by Schemes (2.26) and (2.54) converges to a bounded positive solution $(x, y) \in A(N, M)$ of System (1.14) and has the error estimate (2.7), where $\left\{\beta_{\mu}\right\}_{\mu \in \mathbb{N}_{0}},\left\{\gamma_{\mu}\right\}_{\mu \in \mathbb{N}_{0}} \subset[0,1]$, and $\left\{\left(\delta_{1}^{\mu}, \delta_{2}^{\mu}\right)\right\}_{\mu \in \mathbb{N}_{0}}=\left\{\left(\left\{\delta_{1 n}^{\mu}\right\}_{n \in \mathbb{Z}_{\alpha}},\left\{\delta_{2 n}^{\mu}\right\}_{n \in \mathbb{Z}_{\alpha}}\right)\right\}_{\mu \in \mathbb{N}_{0}} \subset A(N, M)$ are arbitrary sequences with (2.8) and (2.9),

(b) System (1.14) has uncountably many bounded positive solutions in $A(N, M)$.

Theorem 2.13. Assume that there exist $M, N, M_{0}, N_{0}, \bar{p}_{0}, p_{0} \in \mathbb{R}^{+} \backslash\{0\}, n_{1} \in \mathbb{N}_{n_{0}}$ and four nonnegative sequences $\left\{r_{1 n}\right\}_{n \in \mathbb{N}_{n_{0}}},\left\{r_{2 n}\right\}_{n \in \mathbb{N}_{n_{0}}},\left\{t_{1 n}\right\}_{n \in \mathbb{N}_{n_{0}}}$, and $\left\{t_{2 n}\right\}_{n \in \mathbb{N}_{n_{0}}}$ satisfying (2.1), (2.2) with $i=1,(2.24)$, and (2.86)-(2.90). Then,

(a) for each $L \in(N, M) \cap\left(\bar{p}_{0}\left(M_{0} / \underline{p}_{0}+N_{0}\right), \underline{p}_{0}\left(N_{0} / \bar{p}_{0}+M_{0}\right)\right)$, there exist $\theta_{L} \in$ $(0,1)$ and $T_{L} \geq \max \left\{\tau_{1}, \tau_{2}\right\}+n_{1}+|\alpha|$ such that for any $\left(x^{0}, y^{0}\right)=\left(\left\{x_{n}^{0}\right\}_{n \in \mathbb{Z}_{\alpha^{\prime}}}\right.$ $\left.\left\{y_{n}^{0}\right\}_{n \in \mathbb{Z}_{\alpha}}\right) \in A\left(N, M, N_{0}, M_{0}\right)$, the Mann iterative sequence with errors $\left\{\left(x^{\mu}, y^{\mu}\right)\right\}_{\mu \in \mathbb{N}_{0}}=$ $\left\{\left(\left\{x_{n}^{\mu}\right\}_{n \in \mathbb{Z}_{\alpha}},\left\{y_{n}^{\mu}\right\}_{n \in \mathbb{Z}_{\alpha}}\right)\right\}_{\mu \in \mathbb{N}_{0}}$ generated by Schemes (2.26) and (2.67) converges to a bounded positive solution $(x, y) \in A\left(N, M, N_{0}, M_{0}\right)$ of System (1.14) and has the error estimate (2.7), where $\left\{\beta_{\mu}\right\}_{\mu \in \mathbb{N}_{0}},\left\{\gamma_{\mu}\right\}_{\mu \in \mathbb{N}_{0}} \subset[0,1]$, and $\left\{\left(\delta_{1}^{\mu}, \delta_{2}^{\mu}\right)\right\}_{\mu \in \mathbb{N}_{0}}=\left\{\left(\left\{\delta_{1 n}^{\mu}\right\}_{n \in \mathbb{Z}_{\alpha^{\prime}}}\right.\right.$ $\left.\left.\left\{\delta_{2 n}^{\mu}\right\}_{n \in \mathbb{Z}_{\alpha}}\right)\right\}_{\mu \in \mathbb{N}_{0}} \subset A\left(N, M, N_{0}, M_{0}\right)$ are arbitrary sequences with (2.8) and (2.9),

(b) System (1.14) has uncountably many bounded positive solutions in $A\left(N, M, N_{0}, M_{0}\right)$.

Theorem 2.14. Assume that there exist constants $M, N, \bar{p}, \underline{p} \in \mathbb{R}^{+} \backslash\{0\}, \bar{p}_{1}, \underline{p}_{1} \in \mathbb{R}^{+}, n_{1} \in \mathbb{N}_{n_{0}}$ and four nonnegative sequences $\left\{r_{1 n}\right\}_{n \in \mathbb{N}_{n_{0}}},\left\{r_{2 n}\right\}_{n \in \mathbb{N}_{n_{0}}},\left\{t_{1 n}\right\}_{n \in \mathbb{N}_{n_{0}}}$, and $\left\{t_{2 n}\right\}_{n \in \mathbb{N}_{n_{0}}}$ satisfying (2.1), (2.2), (2.24), (2.84),

$$
\begin{gathered}
N<\left(1-\underline{p}_{1}-\bar{p}_{1}\right) M, \quad \underline{p}_{1}+\bar{p}_{1}<1, \quad-\underline{p}_{1} \leq p_{1 n} \leq \bar{p}_{1}, \quad \forall n \geq n_{1}, \\
\max \left\{N+M \bar{p}_{1}, N(\underline{p}-1)\right\}<\min \left\{M\left(1-\underline{p}_{1}\right), M(\bar{p}-1)\right\} .
\end{gathered}
$$


Then,

(a) for each $L \in\left(N+M \bar{p}_{1}, M\left(1-\underline{p}_{1}\right)\right) \cap(N(\underline{p}-1), M(\bar{p}-1))$, there exist $\theta_{L} \in(0,1)$ and $T_{L} \geq \max \left\{\tau_{1}, \tau_{2}\right\}+n_{1}+|\alpha|$ such that for any $\left(x^{0}, y^{0}\right)=\left(\left\{x_{n}^{0}\right\}_{n \in \mathbb{Z}_{\alpha}},\left\{y_{n}^{0}\right\}_{n \in \mathbb{Z}_{\alpha}}\right) \in A(N, M)$, the Mann iterative sequence with errors $\left\{\left(x^{\mu}, y^{\mu}\right)\right\}_{\mu \in \mathbb{N}_{0}}=\left\{\left(\left\{x_{n}^{\mu}\right\}_{n \in \mathbb{Z}_{\alpha}},\left\{y_{n}^{\mu}\right\}_{n \in \mathbb{Z}_{\alpha}}\right)\right\}_{\mu \in \mathbb{N}_{0}}$ generated by Schemes (2.41) and (2.54) converges to a bounded positive solution $(x, y) \in$ $A(N, M)$ of System (1.14) and has the error estimate (2.7), where $\left\{\beta_{\mu}\right\}_{\mu \in \mathbb{N}_{0}},\left\{\gamma_{\mu}\right\}_{\mu \in \mathbb{N}_{0}} \subset$ $[0,1]$, and $\left\{\left(\delta_{1}^{\mu}, \delta_{2}^{\mu}\right)\right\}_{\mu \in \mathbb{N}_{0}}=\left\{\left(\left\{\delta_{1 n}^{\mu}\right\}_{n \in \mathbb{Z}_{\alpha}},\left\{\delta_{2 n}^{\mu}\right\}_{n \in \mathbb{Z}_{\alpha}}\right)\right\}_{\mu \in \mathbb{N}_{0}} \subset A(N, M)$ are arbitrary sequences with (2.8) and (2.9),

(b) System (1.14) has uncountably many bounded positive solutions in $A(N, M)$.

Theorem 2.15. Assume that there exist constants $M, N, M_{0}, N_{0}, \bar{p}_{0}, p_{0}, \bar{p}_{1}, p_{1} \in \mathbb{R}^{+} \backslash\{0\}, n_{1} \in \mathbb{N}_{n_{0}}$ and four nonnegative sequences $\left\{r_{1 n}\right\}_{n \in \mathbb{N}_{n_{0}}},\left\{r_{2 n}\right\}_{n \in \mathbb{N}_{n_{0}}},\left\{t_{1 n}\right\}_{n \in \mathbb{N}_{n_{0}}}$, and $\left\{t_{2 n}\right\}_{n \in \mathbb{N}_{n_{0}}}$ satisfying (2.1), (2.2) with $i=1,(2.24),(2.86)-(2.88),(2.91)$, and

$$
\max \left\{N+M \bar{p}_{1}, \bar{p}_{0}\left(\frac{M_{0}}{\underline{p}_{0}}+N_{0}\right)\right\}<\min \left\{M\left(1-\underline{p}_{1}\right), \underline{p}_{0}\left(\frac{N_{0}}{\bar{p}_{0}}+M_{0}\right)\right\} .
$$

Then,

(a) for each $L \in\left(N+M \bar{p}_{1}, M\left(1-\underline{p}_{1}\right)\right) \cap\left(\bar{p}_{0}\left(M_{0} / \underline{p}_{0}+N_{0}\right), \underline{p}_{0}\left(N_{0} / \bar{p}_{0}+M_{0}\right)\right)$, there exist $\theta_{L} \in(0,1)$ and $T_{L} \geq \max \left\{\tau_{1}, \tau_{2}\right\}+n_{1}+|\alpha|$ such that for any $\left(x^{0}, y^{0}\right)=\left(\left\{x_{n}^{0}\right\}_{n \in \mathbb{Z}_{\alpha}}\right.$ $\left.\left\{y_{n}^{0}\right\}_{n \in \mathbb{Z}_{\alpha}}\right) \in A\left(N, M, N_{0}, M_{0}\right)$, the Mann iterative sequence with errors $\left\{\left(x^{\mu}, y^{\mu}\right)\right\}_{\mu \in \mathbb{N}_{0}}=$ $\left\{\left(\left\{x_{n}^{\mu}\right\}_{n \in \mathbb{Z}_{\alpha}},\left\{y_{n}^{\mu}\right\}_{n \in \mathbb{Z}_{\alpha}}\right)\right\}_{\mu \in \mathbb{N}_{0}}$ generated by Schemes (2.41) and (2.67) converges to a bounded positive solution $(x, y) \in A\left(N, M, N_{0}, M_{0}\right)$ of System (1.14) and has the error estimate (2.7), where $\left\{\beta_{\mu}\right\}_{\mu \in \mathbb{N}_{0}},\left\{\gamma_{\mu}\right\}_{\mu \in \mathbb{N}_{0}} \subset[0,1]$, and $\left\{\left(\delta_{1}^{\mu}, \delta_{2}^{\mu}\right)\right\}_{\mu \in \mathbb{N}_{0}}=\left\{\left(\left\{\delta_{1 n}^{\mu}\right\}_{n \in \mathbb{Z}_{\alpha^{\prime}}}\right.\right.$ $\left.\left.\left\{\delta_{2 n}^{\mu}\right\}_{n \in \mathbb{Z}_{\alpha}}\right)\right\}_{\mu \in \mathbb{N}_{0}} \subset A\left(N, M, N_{0}, M_{0}\right)$ are arbitrary sequences with (2.8) and (2.9)

(b) System (1.14) has uncountably many bounded positive solutions in $A\left(N, M, N_{0}, M_{0}\right)$.

Theorem 2.16. Assume that there exist constants $M, N, M_{0}, N_{0}, \bar{p}_{0}, \underline{p}_{0}, \bar{p}_{1}, \underline{p}_{1} \in \mathbb{R}^{+} \backslash\{0\}, n_{1} \in \mathbb{N}_{n_{0}}$ and four nonnegative sequences $\left\{r_{1 n}\right\}_{n \in \mathbb{N}_{n_{0}}},\left\{r_{2 n}\right\}_{n \in \mathbb{N}_{n_{0}}},\left\{t_{1 n}\right\}_{n \in \mathbb{N}_{n_{0}}}$, and $\left\{t_{2 n}\right\}_{n \in \mathbb{N}_{n_{0}}}$ satisfying (2.1), (2.2) with $i=1,(2.24)$, (2.86)-(2.88),

$$
\begin{gathered}
0<N\left(\underline{p}_{1}-1\right)<M\left(\bar{p}_{1}-1\right), \quad-\underline{p}_{1} \leq p_{1 n} \leq-\bar{p}_{1}, \quad \forall n \geq n_{1}, \\
\max \left\{N\left(\underline{p}_{1}-1\right), \bar{p}_{0}\left(\frac{M_{0}}{\underline{p}_{0}}+N_{0}\right)\right\}<\min \left\{M\left(\bar{p}_{1}-1\right), \underline{p}_{0}\left(\frac{N_{0}}{\bar{p}_{0}}+M_{0}\right)\right\} .
\end{gathered}
$$

Then,

(a) for each $L \in\left(N\left(\underline{p}_{1}-1\right), M\left(\bar{p}_{1}-1\right)\right) \cap\left(\bar{p}_{0}\left(M_{0} / \underline{p}_{0}+N_{0}\right), \underline{p}_{0}\left(N_{0} / \bar{p}_{0}+M_{0}\right)\right)$, there exist $\theta_{L} \in(0,1)$ and $T_{L} \geq \max \left\{\tau_{1}, \tau_{2}\right\}+n_{1}+|\alpha|$ such that for any $\left(x^{0}, y^{0}\right)=\left(\left\{x_{n}^{0}\right\}_{n \in \mathbb{Z}_{\alpha^{\prime}}}\right.$ $\left.\left\{y_{n}^{0}\right\}_{n \in \mathbb{Z}_{\alpha}}\right) \in A\left(N, M, N_{0}, M_{0}\right)$, the Mann iterative sequence with errors $\left\{\left(x^{\mu}, y^{\mu}\right)\right\}_{\mu \in \mathbb{N}_{0}}=$ $\left\{\left(\left\{x_{n}^{\mu}\right\}_{n \in \mathbb{Z}_{\alpha}},\left\{y_{n}^{\mu}\right\}_{n \in \mathbb{Z}_{\alpha}}\right)\right\}_{\mu \in \mathbb{N}_{0}}$ generated by Schemes (2.54) and (2.67) converges to a bounded positive solution $(x, y) \in A\left(N, M, N_{0}, M_{0}\right)$ of System (1.14) and has 
the error estimate (2.7), where $\left\{\beta_{\mu}\right\}_{\mu \in \mathbb{N}_{0}},\left\{\gamma_{\mu}\right\}_{\mu \in \mathbb{N}_{0}} \subset[0,1]$, and $\left\{\left(\delta_{1}^{\mu}, \delta_{2}^{\mu}\right)\right\}_{\mu \in \mathbb{N}_{0}}=$ $\left\{\left(\left\{\delta_{1 n}^{\mu}\right\}_{n \in \mathbb{Z}_{\alpha^{\prime}}}\left\{\delta_{2 n}^{\mu}\right\}_{n \in \mathbb{Z}_{\alpha}}\right)\right\}_{\mu \in \mathbb{N}_{0}} \subset A\left(N, M, N_{0}, M_{0}\right)$ are arbitrary sequences with (2.8) and $(2.9)$,

(b) System (1.14) has uncountably many bounded positive solutions in $A\left(N, M, N_{0}, M_{0}\right)$.

Remark 2.17. Theorems 2.1-2.5 extend and improve [14, Theorem 1] and [27, Theorems 2.12.7].

\section{Examples}

In this section we construct fifteen examples to explain the results presented in Section 2.

Example 3.1. Consider the nonlinear difference system with multiple delays:

$$
\begin{gathered}
\Delta\left(x_{n}-x_{n-\tau_{1}}\right)+\frac{x_{n-3}^{3} y_{n-6}-\sqrt{n} x_{n-4} y_{n-5}^{2}}{n^{3}+n x_{n-3}^{2}+\left(n^{2}+1\right) x_{n-4}^{4} y_{n-6}^{2}}=\frac{(-1)^{n-1}}{n^{4}+\sqrt{n+1}}, \\
\Delta\left(y_{n}-y_{n-\tau_{2}}\right)+\frac{(n+1) x_{n-2}^{2} y_{n-5}+(-1)^{n} y_{n-2}}{n^{4}+(n+2) x_{n-1}^{2}}=\frac{\sqrt{n+1}}{n^{3}+(n+2) \ln n}, \quad n \geq 1,
\end{gathered}
$$

where $\tau_{1}, \tau_{2} \in \mathbb{N}$ are fixed. Let

$$
\begin{gathered}
n_{0}=n_{1}=1, \quad h=k=2, \quad M>N>0, \quad \alpha=\min \left\{1-\tau_{1}, 1-\tau_{2},-5\right\}, \quad a_{1 n}=n-3, \\
a_{2 n}=n-4, \quad b_{1 n}=n-6, \quad b_{2 n}=n-5, \quad c_{1 n}=n-2, \quad c_{2 n}=n-1, \quad d_{1 n}=n-5, \\
d_{2 n}=n-2, \quad p_{1 n}=p_{2 n}=-1, \quad q_{1 n}=\frac{(-1)^{n-1}}{n^{4}+\sqrt{n+1}}, \quad q_{2 n}=\frac{\sqrt{n+1}}{n^{3}+(n+2) \ln n}, \\
f_{1}\left(n, u_{1}, u_{2}, v_{1}, v_{2}\right)=\frac{u_{1}^{3} v_{1}-\sqrt{n} u_{2} v_{2}^{2}}{n^{3}+n u_{1}^{2}+\left(n^{2}+1\right) u_{2}^{4} v_{1}^{2}}, \quad f_{2}\left(n, u_{1}, u_{2}, v_{1}, v_{2}\right)=\frac{(n+1) u_{1}^{2} v_{2}+(-1)^{n} v_{1}}{n^{4}+(n+2) u_{2}^{2}}, \\
r_{1 n}=\frac{M^{3}(M+\sqrt{n})}{n^{3}+n N^{2}+\left(n^{2}+1\right) N^{6}}, \quad r_{2 n}=\frac{M\left(1+(n+1) M^{2}\right)}{n^{4}+(n+2) N^{2}}, \\
t_{1 n}=\frac{n M^{2}\left(4 n^{2} M+3 n^{5 / 2}+2 M^{3}+5 \sqrt{n} M^{2}+16 n M^{7}+14 \sqrt{n} M^{6}\right)}{\left(n^{3}+n N^{2}+\left(n^{2}+1\right) N^{6}\right)^{2}} \\
t_{2 n}=\frac{1}{n^{7}}\left(6 n^{4} M^{2}+n^{3}+30 n M^{4}+9 M^{2}\right), \quad \forall\left(n, u_{1}, u_{2}, v_{1}, v_{2}\right) \in \mathbb{N}_{n_{0}} \times \mathbb{R}^{4} .
\end{gathered}
$$

It is easy to see that (2.1)-(2.4) are satisfied. Thus Theorem 2.1 implies that System (3.1) possesses uncountably many bounded positive solutions in $A(N, M)$. But [14, Theorem 1] and [27, Theorem 2.1] are not valid for System (3.1). 
Example 3.2. Consider the nonlinear difference system with multiple delays:

$$
\begin{gathered}
\Delta\left(x_{n}+x_{n-\tau_{1}}\right)+\frac{n x_{n-1}^{3}-(n-1) y_{n-4}}{n^{3}+x_{n-1}^{2} y_{n-4}^{2}}=\frac{n^{2}-1}{n^{4}+2 n+1}, \\
\Delta\left(y_{n}+y_{n-\tau_{2}}\right)+\frac{n^{9} x_{n-3} y_{n-2}^{2}}{n^{12}+\left(n^{6}-1\right) x_{n-3}^{2}}=\frac{(-1)^{n} n^{2} \ln ^{3} n}{\left(n^{2}+1\right)^{2}}, \quad n \geq 1,
\end{gathered}
$$

where $\tau_{1}, \tau_{2} \in \mathbb{N}$ are fixed. Let

$$
\begin{gathered}
n_{0}=n_{1}=1, \quad h=k=1, \quad M>N>0, \quad \alpha=\min \left\{1-\tau_{1}, 1-\tau_{2},-3\right\}, \quad a_{1 n}=n-1, \\
b_{1 n}=n-4, \quad c_{1 n}=n-3, \quad d_{1 n}=n-2, \quad p_{1 n}=p_{2 n}=1, \quad q_{1 n}=\frac{n^{2}-1}{n^{4}+2 n+1}, \\
q_{2 n}=\frac{(-1)^{n} n^{2} \ln ^{3} n}{\left(n^{2}+1\right)^{2}}, \quad f_{1}(n, u, v)=\frac{n u^{3}-(n-1) v}{n^{3}+u^{2} v^{2}}, \quad f_{2}(n, u, v)=\frac{n^{9} u v^{2}}{n^{12}+\left(n^{6}-1\right) u^{2}}, \\
r_{1 n}=\frac{n M(M+1)}{n^{3}+N^{4}}, \quad r_{2 n}=\frac{n^{9} M^{3}}{n^{12}+\left(n^{6}-1\right) N^{2}}, \quad t_{1 n}=\frac{n\left(n^{3}(2 M+1)+3 M^{4}+2 M^{5}\right)}{\left(n^{3}+N^{4}\right)^{2}}, \\
t_{2 n}=\frac{3 n^{15}\left(n^{6}+1\right) M^{2}}{\left(n^{12}+\left(n^{6}-1\right) N^{2}\right)^{2}}, \quad \forall(n, u, v) \in \mathbb{N}_{n_{0}} \times \mathbb{R}^{2} .
\end{gathered}
$$

It is easy to verify that (2.1), (2.2), (2.24), and (2.25) are fulfilled. Thus Theorem 2.2 implies that System (3.3) possesses uncountably many bounded positive solutions in $A(N, M)$. But in [14, Theorem 1] and [27, Theorem 2.2] are unapplicable for System (3.3).

Example 3.3. Consider the nonlinear difference system with multiple delays:

$$
\begin{gathered}
\Delta\left(x_{n}+\frac{n^{2}}{2 n^{2}+3} x_{n-\tau_{1}}\right)+\frac{x_{n-2}-2 y_{n-6}}{n^{2}+\sin \left(x_{n-1} y_{n-6}\right)}=\frac{\sqrt{n-2} \cos \left(n^{3}-3 n^{2}+6\right)}{n^{3}+3 n+1}, \\
\Delta\left(y_{n}+\frac{(-1)^{n}}{3 n+1} y_{n-\tau_{2}}\right)+\frac{n x_{n-1}+(2 n-1) y_{n-3}^{2}}{n^{3}+x_{n-1}^{2}}=\frac{\left(n^{2}-3\right) \sin \left(n^{5}-2 n+1\right)}{n^{4}+\ln ^{3} n}, \quad n \geq 2,
\end{gathered}
$$


where $\tau_{1}, \tau_{2} \in \mathbb{N}$ are fixed. Let

$$
\begin{aligned}
& n_{0}=n_{1}=2, \quad h=k=1, \quad M>6 N>0, \\
& \alpha=\min \left\{2-\tau_{1}, 2-\tau_{2},-4\right\}, \quad a_{1 n}=n-2, \\
& b_{1 n}=n-6, \quad c_{1 n}=n-1, \quad d_{1 n}=n-3, \quad p_{1 n}=\frac{n^{2}}{2 n^{2}+3}, \\
& p_{2 n}=\frac{(-1)^{n}}{3 n+1}, \quad \bar{p}=\frac{1}{2}, \quad \underline{p}=\frac{1}{3}, \\
& q_{1 n}=\frac{\sqrt{n-2} \cos \left(n^{3}-3 n^{2}+6\right)}{n^{3}+3 n+1}, \quad q_{2 n}=\frac{\left(n^{2}-3\right) \sin \left(n^{5}-2 n+1\right)}{n^{4}+\ln ^{3} n}, \\
& f_{1}(n, u, v)=\frac{u-2 v}{n^{2}+\sin (u v)} \\
& f_{2}(n, u, v)=\frac{n u+(2 n-1) v^{2}}{n^{3}+u^{2}}, \quad r_{1 n}=\frac{3 M}{n^{2}-1}, \quad r_{2 n}=\frac{n M(1+2 M)}{n^{3}+N^{2}}, \\
& t_{1 n}=\frac{6\left(n^{2}+M^{2}\right)}{\left(n^{2}-1\right)^{2}}, \quad t_{2 n}=\frac{n\left((1+4 M) n^{3}+M^{2}+8 M^{3}\right)}{\left(n^{3}+N^{2}\right)^{2}}, \quad \forall(n, u, v) \in \mathbb{N}_{n_{0}} \times \mathbb{R}^{2}
\end{aligned}
$$

It is easy to see that (2.1), (2.2), (2.24), and (2.39) hold. Thus, Theorem 2.3 ensures that System (3.5) possesses uncountably many bounded positive solutions in $A(N, M)$. But [14, Theorem $1]$ and [27, Theorems 2.3, 2.5, and 2.6] are not valid for System (3.5).

Example 3.4. Consider the nonlinear difference system with multiple delays:

$$
\begin{gathered}
\Delta\left(x_{n}+\left(-4+\sin \left(2 n^{2}\right)\right) x_{n-\tau_{1}}\right)+\frac{n^{5} x_{n-1}^{2} y_{n-2}^{5}}{n^{10}+5 n^{8}+1}=\frac{(-1)^{n} n^{3} \ln (1+n)}{n^{9}+n^{5}+1}, \\
\Delta\left(y_{n}+\left(-4+\cos \left(5 n^{3}\right)\right) y_{n-\tau_{2}}\right)+\frac{n^{2} x_{n-2}-(n+2) y_{n-3}^{2}}{n^{8}+1+n x_{n-2}^{2}+y_{n-3}^{2}}=\frac{n^{5}-6}{(n+1)^{7}}, \quad n \geq 1,
\end{gathered}
$$


where $\tau_{1}, \tau_{2} \in \mathbb{N}$ are fixed. Let

$$
\begin{gathered}
n_{0}=n_{1}=h=k=1, \quad M>2 N>0, \quad \alpha=\min \left\{1-\tau_{1}, 1-\tau_{2},-2\right\}, \\
a_{1 n}=n-1, \quad b_{1 n}=n-2, \\
c_{1 n} n-2, \quad d_{1 n}=n-3, \quad p_{1 n}=-4+\sin \left(2 n^{2}\right), \\
p_{2 n}=-4+\cos \left(5 n^{3}\right), \quad \bar{p}=3, \quad \underline{p}=5, \\
q_{1 n}=\frac{(-1)^{n} n^{3} \ln (1+n)}{n^{9}+n^{5}+1}, \quad q_{2 n}=\frac{n^{5}-6}{(n+1)^{7}}, \quad f_{1}(n, u, v)=\frac{n^{5} u^{2} v^{5}}{n^{10}+5 n^{8}+1}, \\
f_{2}(n, u, v)=\frac{n^{2} u-(n+2) v^{2}}{n^{8}+1+n u^{2}+v^{2}}, \quad r_{1 n}=\frac{M^{7}}{n^{5}}, \\
r_{2 n}=\frac{n^{2} M+(n+2) M^{2}}{n^{8}+1+n N^{2}+N^{2}}, \quad t_{1 n}=\frac{7 M^{6}}{n^{5}}, \\
t_{2 n}=\frac{n^{2}\left(2 n^{8}+8 n^{7} M+n M^{2}+8 M^{3}+3 M^{2}\right)}{\left(n^{8}+1+n N^{2}+N^{2}\right)^{2}}, \quad \forall(n, u, v) \in \mathbb{N}_{n_{0}} \times \mathbb{R}^{2} .
\end{gathered}
$$

Clearly (2.1), (2.2), (2.24), and (2.52) hold. Thus, Theorem 2.4 means that System (3.7) possesses uncountably many bounded positive solutions in $A(N, M)$. But [14, Theorem 1] and [27, Theorem 2.4] are inapplicable for System (3.7).

Example 3.5. Consider the nonlinear difference system with multiple delays:

$$
\begin{gathered}
\Delta\left(x_{n}+\frac{8 n^{2}+1}{n^{2}+1} x_{n-\tau_{1}}\right)+\frac{n-(n-1) y_{b_{1 n}}^{3}}{n^{3}+x_{a_{1 n}}^{2}}=\frac{\sqrt{n}-(-1)^{n(n+1) / 2} \sqrt{3 n-2}}{n^{2}+\sqrt{2 n+1}}, \\
\Delta\left(y_{n}+\frac{1+24 \ln ^{3} n}{2+3 \ln ^{3} n} y_{n-\tau_{2}}\right)+\frac{2 n x_{c_{1 n}}-3 \sqrt{n+1} y_{d_{1 n}}^{3}}{n^{4}+x_{c_{1 n}}^{2} y_{d_{1 n}}^{2}}=\frac{1+n \ln \left(1+n^{2}\right)}{n^{3} \ln ^{3}\left(1+n^{2}\right)}, \quad n \geq 1,
\end{gathered}
$$

where $\tau_{1}, \tau_{2} \in \mathbb{N}$ are fixed and

$$
\begin{array}{ll}
a_{1 n}=\left\{\begin{array}{ll}
\frac{n}{2} & \text { if } n \text { is even, } \\
n-2 & \text { if } n \text { is odd, }
\end{array} \quad b_{1 n}= \begin{cases}n-1 & \text { if } n \text { is even, } \\
\frac{n-1}{2} & \text { if } n \text { is odd },\end{cases} \right. \\
c_{1 n}=\left\{\begin{array}{ll}
n-2 & \text { if } n \text { is even, } \\
n-1 & \text { if } n \text { is odd }
\end{array} \quad d_{1 n}= \begin{cases}\frac{n}{2}-1 & \text { if } n \text { is even, } \\
\frac{n+1}{2} & \text { if } n \text { is odd }\end{cases} \right.
\end{array}
$$


Let

$$
\begin{aligned}
& n_{0}=1, \quad n_{1}=10, \quad h=k=1, \quad M>\frac{399}{328} N>0, \\
& \alpha=\min \left\{1-\tau_{1}, 1-\tau_{2},-1\right\}, \quad p_{1 n}=\frac{8 n^{2}+1}{n^{2}+1}, \\
& p_{2 n}=\frac{1+24 \ln ^{3} n}{2+3 \ln ^{3} n}, \quad \bar{p}=8, \quad \underline{p}=7, \\
& q_{1 n}=\frac{\sqrt{n}-(-1)^{n(n+1) / 2} \sqrt{3 n-2}}{n^{2}+\sqrt{2 n+1}}, \quad q_{2 n}=\frac{1+n \ln \left(1+n^{2}\right)}{n^{3} \ln ^{3}\left(1+n^{2}\right)} \text {, } \\
& f_{1}(n, u, v)=\frac{n-(n-1) v^{3}}{n^{3}+u^{2}}, \quad f_{2}(n, u, v)=\frac{2 n u-3 \sqrt{n+1} v^{3}}{n^{4}+u^{2} v^{2}} \text {, } \\
& r_{1 n}=\frac{n\left(1+M^{3}\right)}{n^{3}+N^{2}}, \\
& r_{2 n}=\frac{n M\left(2+3 M^{2}\right)}{n^{4}+N^{4}}, \quad t_{1 n}=\frac{n M\left(2+3 n^{3} M+5 M^{3}\right)}{\left(n^{3}+N^{2}\right)^{2}}, \\
& t_{2 n}=\frac{n\left(2 n^{4}+6 M^{2}+9 n^{4} M^{2}+9 M^{6}\right)}{\left(n^{4}+N^{4}\right)^{2}}, \quad \forall(n, u, v) \in \mathbb{N}_{n_{0}} \times \mathbb{R}^{2} .
\end{aligned}
$$

Obviously (2.1), (2.2), (2.24), and (2.65) hold. Thus, Theorem 2.5 implies that System (3.9) possesses uncountably many bounded positive solutions in $A(N, M)$. But [14, Theorem 1] and [27, Theorem 2.7] are useless for System (3.9).

Example 3.6. Consider the nonlinear difference system with multiple delays:

$$
\begin{gathered}
\Delta\left(x_{n}-x_{n-\tau_{1}}\right)+\frac{2 n^{2} x_{a_{1 n}}^{3}+n-1}{n^{5}+n\left(n x_{a_{1 n}}-(3 n+2) y_{b_{1 n}}\right)^{2}}=\frac{(-1)^{(n-1) n(n+1) / 3} \sqrt{2 n^{3}+4}}{n^{4}+\sin ^{2}\left(2 n^{4}+1\right)}, \\
\Delta\left(y_{n}+y_{n-\tau_{2}}\right)+\frac{(-1)^{n} x_{c_{1 n}}^{2}-n y_{d_{1 n}}^{3}}{(n+1)^{2}(\sqrt{n}+1)}=\frac{(-1)^{n-1} \ln \left(n^{3}+2 n+1\right)}{n^{3 / 2}+\ln ^{3}\left(1+n^{2}\right)}, \quad n \geq 1,
\end{gathered}
$$

where $\tau_{1}, \tau_{2} \in \mathbb{N}$ are fixed and

$$
\begin{array}{ll}
a_{1 n}=\left\{\begin{array}{ll}
n-1 & \text { if } n \geq 100, \\
n-2 & \text { if } 1 \leq n<100,
\end{array} \quad b_{1 n}= \begin{cases}\frac{n}{2} & \text { if } n \text { is even, } \\
\frac{n-1}{2} & \text { if } n \text { is odd },\end{cases} \right. \\
c_{1 n}=\left\{\begin{array}{ll}
\frac{n}{2}-2 & \text { if } n \text { is even, } \\
\frac{n-1}{2} & \text { if } n \text { is odd },
\end{array} \quad d_{1 n}= \begin{cases}n-3 & \text { if } n \geq 100, \\
n & \text { if } 1 \leq n<100 .\end{cases} \right.
\end{array}
$$


Let

$$
\begin{aligned}
& n_{0}=n_{1}=h=k=1, \quad M>N>0, \quad \alpha=\min \left\{1-\tau_{1}, 1-\tau_{2},-1\right\}, \quad p_{1 n}=-1, \\
& p_{2 n}=1, \quad q_{1 n}=\frac{(-1)^{(n-1) n(n+1) / 3} \sqrt{2 n^{3}+4}}{n^{4}+\sin ^{2}\left(2 n^{4}+1\right)}, \quad q_{2 n}=\frac{(-1)^{n-1} \ln \left(n^{3}+2 n+1\right)}{n^{3 / 2}+\ln ^{3}\left(1+n^{2}\right)} \\
& f_{1}(n, u, v)=\frac{2 n^{2} u^{3}+n-1}{n^{5}+n(n u-(3 n+2) v)^{2}}, \quad f_{2}(n, u, v)=\frac{(-1)^{n} u^{2}-n v^{3}}{(n+1)^{2}(\sqrt{n}+1)}, \\
& r_{1 n}=\frac{1+2 n M^{3}}{n^{4}}, \quad r_{2 n}=\frac{M^{2}+n M^{3}}{n^{5 / 2}}, \\
& t_{1 n}=\frac{6 M\left(16+n^{3} M+8(2 n+M) M^{2}+3 n M^{3}\right)}{n^{6}}, \\
& t_{2 n}=\frac{2 M+3 n M^{2}}{n^{5 / 2}}, \quad \forall(n, u, v) \in \mathbb{N}_{n_{0}} \times \mathbb{R}^{2} .
\end{aligned}
$$

It is easy to see that (2.1), (2.2), and (2.79)-(2.82) are satisfied. Thus, Theorem 2.7 implies that System (3.12) possesses uncountably many bounded positive solutions in $A(\mathrm{~N}, M)$.

Example 3.7. Consider the nonlinear difference system with multiple delays:

$$
\begin{gathered}
\Delta\left(x_{n}-x_{n-\tau_{1}}\right)+\frac{n x_{a_{1 n}}^{3}-y_{b_{1 n}}}{(n+1)^{4}+(\sqrt{n}+1) y_{b_{1 n}}^{2}}=\frac{(-1)^{n(n+1) / 2}(1-\sqrt{3 n+1})}{n^{3}+\sqrt{3 n+2}}, \\
\Delta\left(y_{n}+\frac{n^{2}-1}{3 n^{2}+2} y_{n-\tau_{2}}\right)+\frac{\sqrt{n}(n+1) y_{d_{1 n}}^{4}}{(n+2)^{3}+n x_{c_{1 n}}^{2}}=\frac{\sqrt{2 n+1}(n+3)^{2} \ln \left(1+n^{4}\right)}{(n+1)^{4}}, \quad n \geq 1,
\end{gathered}
$$

where $\tau_{1}, \tau_{2} \in \mathbb{N}$ are fixed and

$$
\begin{array}{ll}
a_{1 n}= \begin{cases}n-4 & \text { if } n \text { is even, } \\
n-1 & \text { if } n \text { is odd, }\end{cases} & b_{1 n}= \begin{cases}n-2 & \text { if } n \text { is even, } \\
\frac{n-1}{2} & \text { if } n \text { is odd, }\end{cases} \\
c_{1 n}=\left\{\begin{array}{ll}
n-1 & \text { if } n \text { is even, } \\
\frac{n+1}{2} & \text { if } n \text { is odd, }
\end{array} \quad d_{1 n}= \begin{cases}\frac{n}{2} & \text { if } n \text { is even, } \\
n-2 & \text { if } n \text { is odd. }\end{cases} \right.
\end{array}
$$


Let

$$
\begin{gathered}
n_{0}=n_{1}=h=k=1, \quad M>6 N>0, \quad \alpha=\min \left\{1-\tau_{1}, 1-\tau_{2},-2\right\}, \\
p_{1 n}=-1, \quad p_{2 n}=\frac{n^{2}-1}{3 n^{2}+2^{\prime}}, \\
\bar{p}=\frac{1}{3}, \quad \underline{p}=\frac{1}{2}, \quad q_{1 n}=\frac{(-1)^{n(n+1) / 2}(1-\sqrt{3 n+1})}{n^{3}+\sqrt{3 n+2}}, \\
q_{2 n}=\frac{\sqrt{2 n+1}(n+3)^{2} \ln \left(1+n^{4}\right)}{(n+1)^{4}}, \\
f_{1}(n, u, v)=\frac{n u^{3}-v \quad f_{2}(n, u, v)=\frac{\sqrt{n}(n+1) v^{4}}{(n+1)^{4}+(\sqrt{n}+1) v^{2}},}{\left(n u^{2}\right.}, \\
r_{2 n}=\frac{r_{1 n}=\frac{\left(n M^{2}+1\right) M}{(n+1)^{4}},}{(n+2)^{3}+n N^{2}}, \quad t_{1 n}=\frac{(n+1)^{4}\left(3 n M^{2}+1\right)+\left(5 n M^{2}+1\right)(\sqrt{n}+1) M^{2}}{\left((n+1)^{4}+(\sqrt{n}+1) N^{2}\right)^{2}}, \\
t_{2 n}=\frac{2 \sqrt{n}(n+1) M^{3}\left(2(n+2)^{3}+3 n M^{2}\right)}{\left((n+2)^{3}+n N^{2}\right)^{2}}, \quad \forall(n, u, v) \in \mathbb{N}_{n_{0}} \times \mathbb{R}^{2} .
\end{gathered}
$$

Clearly (2.1), (2.2), (2.79)-(2.81), and (2.83) are satisfied. Thus, Theorem 2.8 guarantees that System (3.15) possesses uncountably many bounded positive solutions in $A(N, M)$.

Example 3.8. Consider the nonlinear difference system with multiple delays:

$$
\begin{gathered}
\Delta\left(x_{n}-x_{n-\tau_{1}}\right)+\frac{n^{7}-x_{a_{1 n}}^{2}}{n^{10}+2 y_{b_{1 n}}^{2}}=\frac{n^{20}-(-1)^{(n+1)(n+2) / 2}\left(3 n^{5}+1\right)^{2}}{n^{23}+(n+5)^{5} \ln ^{8}\left(n^{2}+1\right)}, \\
\Delta\left(y_{n}-\frac{6 n^{5}}{2 n^{5}+1} y_{n-\tau_{2}}\right)+\frac{n^{3} x_{c_{1 n}} y_{d_{1 n}}^{2}}{n^{6}+(n+1) x_{c_{1 n}}^{4}}=\frac{n^{8}-(-1)^{n-1}\left(n^{3}+1\right)^{2}}{(n+1)^{11}}, \quad n \geq 2,
\end{gathered}
$$

where $\tau_{1}, \tau_{2} \in \mathbb{N}$ are fixed and

$$
\begin{aligned}
& a_{1 n}=\left\{\begin{array}{ll}
\frac{n}{2}-1 & \text { if } n \text { is even, } \\
\frac{n-3}{2} & \text { if } n \text { is odd, }
\end{array} \quad b_{1 n}= \begin{cases}n-1 & \text { if } n \text { is even, } \\
n-2 & \text { if } n \text { is odd, }\end{cases} \right. \\
& c_{1 n}=\left\{\begin{array}{ll}
\frac{n}{2}+1 & \text { if } n \text { is even, } \\
\frac{n+1}{2} & \text { if } n \text { is odd, }
\end{array} \quad d_{1 n}= \begin{cases}n-5 & \text { if } n \text { is even, } \\
n-3 & \text { if } n \text { is odd. }\end{cases} \right.
\end{aligned}
$$


Let

$$
\begin{gathered}
n_{0}=n_{1}=2, \quad h=k=1, \quad M>6 N>0, \quad \alpha=\min \left\{2-\tau_{1}, 2-\tau_{2},-3\right\}, \quad p_{1 n}=-1, \\
p_{2 n}=-\frac{6 n^{5}}{2 n^{5}+1}, \quad \quad \quad \quad=2, \quad \quad \underline{p}=3, \quad q_{1 n}=\frac{n^{20}-(-1)^{(n+1)(n+2) / 2}\left(3 n^{5}+1\right)^{2}}{n^{23}+(n+5)^{5} \ln ^{8}\left(n^{2}+1\right)}, \\
q_{2 n}=\frac{n^{8}-(-1)^{n-1}\left(n^{3}+1\right)^{2}}{(n+1)^{11}}, \quad f_{1}(n, u, v)=\frac{n^{7}-u^{2}}{n^{10}+2 v^{2}}, \quad f_{2}(n, u, v)=\frac{n^{3} u v^{2}}{n^{6}+(n+1) u^{4}}, \\
r_{1 n}=\frac{n^{7}+M^{2}}{n^{10}+2 N^{2}}, \quad r_{2 n}=\frac{n^{3} M^{3}}{n^{6}+(n+1) N^{4}}, \quad t_{1 n}=\frac{2 M\left(2 n^{7}+n^{10}+4 M^{2}\right)}{\left(n^{10}+2 N^{2}\right)^{2}}, \\
t_{2 n}=\frac{3 n^{3} M^{2}\left(n^{6}+(n+1) M^{4}\right)}{\left(n^{6}+(n+1) N^{4}\right)^{2}}, \quad \forall(n, u, v) \in \mathbb{N}_{n_{0}} \times \mathbb{R}^{2} .
\end{gathered}
$$

Obviously (2.1), (2.2), (2.79)-(2.81), (2.84), and (2.85) hold. Thus, Theorem 2.9 implies that System (3.18) possesses uncountably many bounded positive solutions in $A(N, M)$.

Example 3.9. Consider the nonlinear difference system with multiple delays:

$$
\begin{gathered}
\Delta\left(x_{n}-x_{n-\tau_{1}}\right)+\frac{n x_{a_{1 n}}^{2}-y_{b_{1 n}}^{3}}{n^{3} \ln ^{2} n+1}=\frac{n^{2}-(-1)^{n-1}(3 n+1)}{n^{5}+8(n+1)^{4}+1}, \\
\Delta\left(y_{n}+\frac{8 n^{2}}{2 n^{2}+3} y_{n-\tau_{2}}\right)+\frac{n^{2}}{n^{4}+\left|x_{c_{1 n}} y_{d_{1 n}}\right|}=\frac{(-1)^{n-1} \sin \left(n^{5}+1\right)}{n^{2}+1}, \quad n \geq 1,
\end{gathered}
$$

where $\tau_{1}, \tau_{2} \in \mathbb{N}$ are fixed and

$$
\begin{array}{ll}
a_{1 n}= \begin{cases}n-2 & \text { if } n \text { is even, } \\
\frac{n-3}{2} & \text { if } n \text { is odd, }\end{cases} & b_{1 n}= \begin{cases}\frac{n}{2} & \text { if } n \text { is even, } \\
\frac{n+1}{2} & \text { if } n \text { is odd, }\end{cases} \\
c_{1 n}=\left\{\begin{array}{ll}
n-1 & \text { if } n \text { is even, } \\
\frac{n-1}{2} & \text { if } n \text { is odd, }
\end{array} \quad d_{1 n}= \begin{cases}n-4 & \text { if } n \text { is even, } \\
n-2 & \text { if } n \text { is odd. }\end{cases} \right.
\end{array}
$$


Let

$$
\begin{gathered}
n_{0}=1, \quad n_{1}=3, \quad h=k=1, \quad M=3000, \\
N=2000, \quad M_{0}=1200, \quad N_{0}=40, \\
\alpha=\min \left\{1-\tau_{1}, 1-\tau_{2},-2\right\}, \quad p_{1 n}=-1, \quad p_{2 n}=\frac{8 n^{2}}{2 n^{2}+3}, \quad \bar{p}_{0}=4, \quad \underline{p}_{0}=3, \\
q_{1 n}=\frac{n^{2}-(-1)^{n-1}(3 n+1)}{n^{5}+8(n+1)^{4}+1}, \quad q_{2 n}=\frac{(-1)^{n-1} \sin \left(n^{5}+1\right)}{n^{2}+1}, \quad f_{1}(n, u, v)=\frac{n u^{2}-v^{3}}{n^{3} \ln ^{2} n+1}, \\
f_{2}(n, u, v)=\frac{n^{2}}{n^{4}+|u v|}, \quad r_{1 n}=\frac{M^{2}(n+M)}{n^{3} \ln ^{2} n+1}, \quad r_{2 n}=\frac{n^{2}}{n^{4}+N_{0}^{2}}, \\
t_{1 n}=\frac{M(2 n+3 M)}{n^{3} \ln ^{2} n+1}, \quad t_{2 n}=\frac{2 n^{2} M_{0}}{\left(n^{4}+N_{0}^{2}\right)^{2}}, \quad \forall(n, u, v) \in \mathbb{N}_{n_{0}} \times \mathbb{R}^{2} .
\end{gathered}
$$

It is easy to verify that (2.1), (2.2) with $i=1,(2.79)-(2.81)$, and (2.86)-(2.89) are satisfied. Thus, Theorem 2.10 reveals that System (3.21) possesses uncountably many bounded positive solutions in $A\left(N, M, N_{0}, M_{0}\right)$.

Example 3.10. Consider the nonlinear difference system with multiple delays:

$$
\begin{gathered}
\Delta\left(x_{n}+x_{n-\tau_{1}}\right)+\frac{1-n^{5} x_{a_{1 n}}^{2}}{n^{7}+(n+3) \ln \left(1+y_{b_{1 n}}^{2}\right)}=\frac{n^{4}-4 n^{3}+n-1}{n^{6}+(n-1)^{4}}, \\
\Delta\left(y_{n}-\frac{2 n^{2}}{5 n^{2}+6} y_{n-\tau_{2}}\right)+\frac{n^{2} y_{d_{1 n}}}{n^{4}+\sin ^{2}\left(x_{c_{1 n}}^{2}+n y_{d_{1 n}}^{2}\right)}=\frac{(-1)^{n} \sqrt{n-1}}{n^{3}+2 n^{2}+1}, \quad n \geq 2,
\end{gathered}
$$

where $\tau_{1}, \tau_{2} \in \mathbb{N}$ are fixed and

$$
\begin{array}{ll}
a_{1 n}= \begin{cases}n-3 & \text { if } n \text { is even, } \\
\frac{n-1}{2} & \text { if } n \text { is odd, }\end{cases} & b_{1 n}= \begin{cases}\frac{n}{2}+1 & \text { if } n \text { is even, } \\
\frac{n-1}{2} & \text { if } n \text { is odd, }\end{cases} \\
c_{1 n}=\left\{\begin{array}{ll}
n-1 & \text { if } n \text { is even, } \\
\frac{n+1}{2} & \text { if } n \text { is odd, }
\end{array} \quad d_{1 n}= \begin{cases}n-2 & \text { if } n \text { is even, } \\
\frac{n+1}{2} & \text { if } n \text { is odd. }\end{cases} \right.
\end{array}
$$


Let

$$
\begin{gathered}
n_{0}=n_{1}=2, \quad h=k=1, \quad M>\frac{5 N}{3}>0, \quad \alpha=\min \left\{1-\tau_{1}, 1-\tau_{2},-1\right\}, \quad p_{1 n}=1, \\
p_{2 n}=-\frac{2 n^{2}}{5 n^{2}+6}, \quad \bar{p}=0, \quad \quad \underline{p}=\frac{2}{5}, \quad q_{1 n}=\frac{n^{4}-4 n^{3}+n-1}{n^{6}+(n-1)^{4}}, \quad q_{2 n}=\frac{(-1)^{n} \sqrt{n-1}}{n^{3}+2 n^{2}+1}, \\
f_{1}(n, u, v)=\frac{1-n^{5} u^{2}}{n^{7}+(n+3) \ln \left(1+v^{2}\right)}, \quad f_{2}(n, u, v)=\frac{n^{2} v}{n^{4}+\sin ^{2}\left(u^{2}+n v^{2}\right)}, \\
r_{1 n}=\frac{1+n^{5} M^{2}}{n^{7}+(n+3) \ln \left(1+N^{2}\right)}, \quad r_{2 n}=\frac{M}{n^{2}}, \\
\frac{1}{\left(n^{7}+(n+3) \ln \left(1+N^{2}\right)\right)^{2}}\left[\frac{2 \mathrm{M}(n+3)}{1+N^{2}}+n^{12}+n^{5}(n+3)\left(\frac{2 M^{2}}{1+N^{2}}+\ln \left(1+M^{2}\right)\right)\right] \\
t_{2 n}=\frac{n^{4}+4 n M^{2}+4 M^{2}+1}{n^{6}}, \quad \forall(n, u, v) \in \mathbb{N}_{n_{0}} \times \mathbb{R}^{2} .
\end{gathered}
$$

Obviously (2.1), (2.2), (2.24), (2.83), and (2.90) hold. Thus, Theorem 2.11 gives that System (3.24) possesses uncountably many bounded positive solutions in $A(N, M)$.

Example 3.11. Consider the nonlinear difference system with multiple delays:

$$
\begin{gathered}
\Delta\left(x_{n}+x_{n-\tau_{1}}\right)+\frac{n^{2}-x_{a_{1 n}}^{2} y_{b_{1 n}}}{n^{4}+(n+1) y_{b_{1 n}}^{4}}=\frac{n^{6}-9 n^{5}+(-1)^{n} n^{4}-n^{3}+n-1}{n^{8}+3 n^{7}+n^{5}+4 n^{4}+n^{2}+1}, \\
\Delta\left(y_{n}-\frac{3 n^{3}}{n^{3}+1} y_{n-\tau_{2}}\right)+\frac{n y_{d_{1 n}}^{2}}{n^{3}+x_{c_{1 n}}^{2}}=\frac{(-1)^{n(n+1) / 2}(2 n-5) \ln ^{2}\left(1+n^{2}\right)}{n^{3}+3 n^{2}+5 n+1}, \quad n \geq 1,
\end{gathered}
$$

where $\tau_{1}, \tau_{2} \in \mathbb{N}$ are fixed and

$$
\begin{array}{ll}
a_{1 n}= \begin{cases}n-2 & \text { if } n \text { is even, } \\
\frac{n+1}{2} & \text { if } n \text { is odd, }\end{cases} & b_{1 n}= \begin{cases}\frac{n}{2}-1 & \text { if } n \text { is even, } \\
\frac{n+1}{2} & \text { if } n \text { is odd, }\end{cases} \\
c_{1 n}=\left\{\begin{array}{ll}
n-3 & \text { if } n \text { is even, } \\
\frac{n-3}{2} & \text { if } n \text { is odd, }
\end{array} \quad d_{1 n}= \begin{cases}n-4 & \text { if } n \text { is even, } \\
\frac{n-1}{2} & \text { if } n \text { is odd. }\end{cases} \right.
\end{array}
$$


Let

$$
\begin{gathered}
n_{0}=n_{1}=h=k=1, \quad M>4 N>0, \quad \alpha=\min \left\{1-\tau_{1}, 1-\tau_{2},-2\right\}, \quad p_{1 n}=1, \\
p_{2 n}=-\frac{3 n^{3}}{n^{3}+1}, \quad \bar{p}=\frac{3}{2}, \quad \underline{p}=3, \quad q_{1 n}=\frac{n^{6}-9 n^{5}+(-1)^{n} n^{4}-n^{3}+n-1}{n^{8}+3 n^{7}+n^{5}+4 n^{4}+n^{2}+1}, \\
q_{2 n}=\frac{(-1)^{n(n+1) / 2}(2 n-5) \ln ^{2}\left(1+n^{2}\right)}{n^{3}+3 n^{2}+5 n+1}, \quad f_{1}(n, u, v)=\frac{n^{2}-u^{2} v}{n^{4}+(n+1) v^{4}}, \\
f_{2}(n, u, v)=\frac{n v^{2}}{n^{3}+u^{2}}, \quad r_{1 n}=\frac{n^{2}+M^{3}}{n^{4}+(n+1) N^{4}}, \quad r_{2 n}=\frac{n M^{2}}{n^{3}+N^{2}}, \\
t_{1 n}=\frac{n M^{2}\left(8 n^{2} M+3 n^{3}+10 M^{4}\right)}{\left(n^{4}+(n+1) N^{4}\right)^{2}}, \quad t_{2 n}=\frac{2 M\left(n^{3}+2 M^{2}\right)}{\left(n^{3}+N^{2}\right)^{2}}, \quad \forall(n, u, v) \in \mathbb{N}_{n_{0}} \times \mathbb{R}^{2} .
\end{gathered}
$$

Clearly (2.1), (2.2), (2.24), (2.84), (2.85), and (2.90) hold. Thus, Theorem 2.12 ensures that System (3.27) possesses uncountably many bounded positive solutions in $A(N, M)$.

Example 3.12. Consider the nonlinear difference system with multiple delays:

$$
\begin{gathered}
\Delta\left(x_{n}+x_{n-\tau_{1}}\right)+\frac{n^{25}-\left(n^{15}+1\right) y_{n-1}^{2}}{n^{30}+\left(n^{18}+1\right) x_{n-2}^{2}}=\frac{n^{38}-19 n^{25}+30 n^{18}-3 n^{9}+2 n-1}{n^{40}+8 n^{30}+n^{15}+6 n^{2}+1}, \\
\Delta\left(y_{n}+\frac{16 n^{3}+1}{4 n^{3}+131} y_{n-\tau_{2}}\right)+\frac{x_{n-4}^{3} y_{n-3}^{2}}{n \ln ^{10} n+1}=\frac{n^{34}-6 n^{32}-7 n^{15}+(-1)^{n} n^{7}-1}{n^{36}+6 n^{30}+5 n^{12}+6 n^{6}+n^{3}+1}, \quad n \geq 2,
\end{gathered}
$$

where $\tau_{1}, \tau_{2} \in \mathbb{N}$ are fixed. Let

$$
\begin{gathered}
n_{0}=2, \quad n_{1}=4, \quad h=k=1, \quad M=800 N_{0}, \\
N=500 N_{0}, \quad M_{0}=300 N_{0}>0, \\
\alpha=\min \left\{2-\tau_{1}, 2-\tau_{2},-2\right\}, \quad a_{1 n}=n-2, \quad b_{1 n}=n-1, \\
c_{1 n}=n-4, \quad d_{1 n}=n-3,
\end{gathered}
$$


Abstract and Applied Analysis

$$
\begin{gathered}
p_{1 n}=1, \quad p_{2 n}=\frac{16 n^{3}+1}{4 n^{3}+131}, \quad \bar{p}_{0}=4, \quad \underline{p}_{0}=3, \\
q_{1 n}=\frac{n^{38}-19 n^{25}+30 n^{18}-3 n^{9}+2 n-1}{n^{40}+8 n^{30}+n^{15}+6 n^{2}+1}, \\
q_{2 n}=\frac{n^{34}-6 n^{32}-7 n^{15}+(-1)^{n} n^{7}-1}{n^{36}+6 n^{30}+5 n^{12}+6 n^{6}+n^{3}+1}, \quad f_{1}(n, u, v)=\frac{n^{25}-\left(n^{15}+1\right) v^{2}}{n^{30}+\left(n^{18}+1\right) u^{2}} \\
f_{2}(n, u, v)=\frac{u^{3} v^{2}}{n \ln ^{10} n+1}, \quad r_{1 n}=\frac{n^{25}+\left(n^{15}+1\right) M^{2}}{n^{30}+\left(n^{18}+1\right) N^{2}}, \quad r_{2 n}=\frac{M_{0}^{5}}{n \ln ^{10} n+1}, \\
t_{1 n}=\frac{4 n^{33} M\left(n^{10}+n^{12}+4 M^{2}\right)}{\left(n^{30}+\left(n^{18}+1\right) N^{2}\right)^{2}}, \quad t_{2 n}=\frac{5 M_{0}^{4}}{\left(n \ln ^{10} n+1\right)^{2}}, \quad \forall(n, u, v) \in \mathbb{N}_{n_{0}} \times \mathbb{R}^{2} .
\end{gathered}
$$

It is easy to verify that (2.1), (2.2) with $i=1,(2.24)$, and (2.86)-(2.90) hold. Thus, Theorem 2.13 guarantees that System (3.30) possesses uncountably many bounded positive solutions in $A\left(N, M, N_{0}, M_{0}\right)$.

Example 3.13. Consider the nonlinear difference system with multiple delays:

$$
\begin{gathered}
\Delta\left(x_{n}+\frac{3(-1)^{n} n^{10}+2 n+1}{9 n^{10}+4 n^{8}+1} x_{n-\tau_{1}}\right)+\frac{x_{n-3}-n y_{n-1}}{n^{4}+x_{n-3}^{2}}=\frac{n^{4}-\sqrt{2 n+5} \ln ^{5} n}{n^{6}+6 n^{2}+1}, \\
\Delta\left(y_{n}-\frac{\left(4+(-1)^{n}\right) n^{4}+5}{n^{4}+1} y_{n-\tau_{2}}\right)+\frac{n^{3} x_{n-2} y_{n-4}}{n^{5}+(n+1) y_{n-4}^{2}}=\frac{n^{7}-(-1)^{n} n^{4}-1}{n^{9}+3 n^{6}+5}, \quad n \geq 2,
\end{gathered}
$$

where $\tau_{1}, \tau_{2} \in \mathbb{N}$ are fixed. Let

$$
\begin{gathered}
n_{0}=2, \quad n_{1}=4, \quad h=k=1, \quad M>10 N>0, \quad \alpha=\min \left\{2-\tau_{1}, 2-\tau_{2},-2\right\}, \\
a_{1 n}=n-3, \quad b_{1 n}=n-1, \quad c_{1 n}=n-2, \quad d_{1 n}=n-4, \quad p_{1 n}=\frac{3(-1)^{n} n^{10}+2 n+1}{9 n^{10}+4 n^{8}+1}, \\
p_{2 n}=-\frac{\left(4+(-1)^{n}\right) n^{4}+5}{n^{4}+1}, \quad \bar{p}_{1}=\underline{p}_{1}=\frac{1}{3}, \quad \bar{p}=3, \quad \underline{p}=5, \quad q_{1 n}=\frac{n^{4}-\sqrt{2 n+5} \ln ^{5} n}{n^{6}+6 n^{2}+1}, \\
q_{2 n}=\frac{n^{7}-(-1)^{n} n^{4}-1}{n^{9}+3 n^{6}+5}, \quad f_{1}(n, u, v)=\frac{u-n v}{n^{4}+u^{2}}, \quad f_{2}(n, u, v)=\frac{n^{3} u v}{n^{5}+(n+1) v^{2}}, \\
r_{1 n}=\frac{M(n+1)}{n^{4}+N^{2}}, \quad r_{2 n}=\frac{n^{3} M^{2}}{n^{5}+(n+1) N^{2}}, \quad t_{1 n}=\frac{n^{4}+M^{2}+n^{5}+3 n M^{2}}{\left(n^{4}+N^{2}\right)^{2}}, \\
t_{2 n}=\frac{2 n^{3} M\left(n^{5}+(n+1) M^{2}\right)}{\left(n^{5}+(n+1) N^{2}\right)^{2}}, \quad \forall(n, u, v) \in \mathbb{N}_{n_{0}} \times \mathbb{R}^{2} .
\end{gathered}
$$


Obviously (2.1), (2.2), (2.24), (2.84), (2.91), and (2.92) hold. Thus, Theorem 2.14 means that System (3.32) possesses uncountably many bounded positive solutions in $A(N, M)$.

Example 3.14. Consider the nonlinear difference system with multiple delays:

$$
\begin{gathered}
\Delta\left(x_{n}+\frac{(-1)^{n} n+1}{4 n+100} x_{n-\tau_{1}}\right)+\frac{x_{n-2}-n y_{n-1}}{n^{3}+x_{n-2}^{2} y_{n-1}^{2}}=\frac{n^{5} \ln ^{9} n-(-1)^{n}}{n^{8}+5 n^{7}+1}, \\
\Delta\left(y_{n}+\frac{64 n^{8}+1}{8 n^{8}+3 n^{4}+7} y_{n-\tau_{2}}\right)+\frac{n x_{n-5}^{2}-y_{n-3} \sin n}{n^{2} \ln ^{2} n+1}=\frac{n^{6}-(-1)^{n} n^{2}+1}{n^{8}+7 n^{7}+2}, \quad n \geq 2,
\end{gathered}
$$

where $\tau_{1}, \tau_{2} \in \mathbb{N}$ are fixed. Let

$$
\begin{gathered}
n_{0}=n_{1}=2, \quad h=k=1, \quad M=40000, \quad N=5000, \quad M_{0}=4480, \quad N_{0}=80, \\
\alpha=\min \left\{2-\tau_{1}, 2-\tau_{2},-3\right\}, \quad a_{1 n}=n-2, \quad b_{1 n}=n-1, \quad c_{1 n}=n-5, \quad d_{1 n}=n-3, \\
p_{1 n}=\frac{(-1)^{n} n+1}{4 n+100}, \quad p_{2 n}=\frac{64 n^{8}+1}{8 n^{8}+3 n^{4}+7}, \quad \bar{p}_{1}=\underline{p}_{1}=\frac{1}{4}, \quad \bar{p}_{0}=8, \quad \underline{p}_{0}=7, \\
q_{1 n}=\frac{n^{5} \ln ^{9} n-(-1)^{n}}{n^{8}+5 n^{7}+1}, \quad q_{2 n}=\frac{n^{6}-(-1)^{n} n^{2}+1}{n^{8}+7 n^{7}+2}, \quad f_{1}(n, u, v)=\frac{u-n v}{n^{3}+u^{2} v^{2}}, \\
f_{2}(n, u, v)=\frac{n u^{2}-v \sin n}{n^{2} \ln ^{2} n+1}, \quad r_{1 n}=\frac{M(n+1)}{n^{3}+N^{4}}, \quad r_{2 n}=\frac{M_{0}\left(M_{0}+1\right)}{n^{2} \ln ^{2} n+1}, \\
t_{1 n}=\frac{n^{3}+3 M^{4}+n^{4}+3 n M^{2}}{\left(n^{3}+N^{4}\right)^{2}}, \quad t_{2 n}=\frac{2 M_{0}+1}{\left(n^{2} \ln ^{2} n+1\right)^{2}}, \quad \forall(n, u, v) \in \mathbb{N}_{n_{0}} \times \mathbb{R}^{2} .
\end{gathered}
$$

It is easy to see that (2.1), (2.2) with $i=1,(2.24),(2.86)-(2.88),(2.91)$, and (2.93) hold. Thus, Theorem 2.15 implies that System (3.34) possesses uncountably many bounded positive solutions in $A\left(N, M, N_{0}, M_{0}\right)$.

Example 3.15. Consider the nonlinear difference system with multiple delays:

$$
\begin{gathered}
\Delta\left(x_{n}+\frac{-20 n+1}{4 n+9} x_{n-\tau_{1}}\right)+\frac{n x_{n-1}^{3}}{n^{3}+y_{n-2}^{2}}=\frac{\sqrt{n^{3}+3 n^{2} \sqrt{n-1}+1}}{n^{5}+3 n^{4}+1}, \\
\Delta\left(y_{n}+\frac{6 n^{2}+1}{2 n^{2}+5 n+3} y_{n-\tau_{2}}\right)+\frac{x_{n-2} y_{n-4}}{n^{2}+\cos \left(n^{2}+1\right)}=\frac{n^{4}+5 n^{2}-7}{n^{6}+4 n^{3}+1}, \quad n \geq 2,
\end{gathered}
$$


where $\tau_{1}, \tau_{2} \in \mathbb{N}$ are fixed. Let

$$
\begin{gathered}
n_{0}=2, \quad n_{1}=10, \quad h=k=1, \quad M=2 N_{0}, \quad N=N_{0}>0, \quad M_{0}=30 N_{0}, \\
\alpha=\min \left\{2-\tau_{1}, 2-\tau_{2},-2\right\}, \quad a_{1 n}=n-1, \quad b_{1 n}=n-2, \quad c_{1 n}=n-2, \quad d_{1 n}=n-4, \\
p_{1 n}=\frac{-20 n+1}{4 n+9}, \quad p_{2 n}=\frac{6 n^{2}+1}{2 n^{2}+5 n+3}, \quad \bar{p}_{1}=4, \quad \underline{p}_{1}=5, \quad \bar{p}_{0}=3, \quad \underline{p}_{0}=2, \\
q_{1 n}=\frac{\sqrt{n^{3}+3 n^{2} \sqrt{n-1}+1}}{n^{5}+3 n^{4}+1}, \quad q_{2 n}=\frac{n^{4}+5 n^{2}-7}{n^{6}+4 n^{3}+1}, \quad f_{1}(n, u, v)=\frac{n u^{3}}{n^{3}+v^{2}}, \\
f_{2}(n, u, v)=\frac{u v}{n^{2}+\cos \left(n^{2}+1\right)}, \quad r_{1 n}=\frac{n M^{3}}{n^{3}+N^{2}}, \quad r_{2 n}=\frac{M_{0}^{2}}{n^{2}-1}, \\
t_{1 n}=\frac{n M^{2}\left(3 n^{3}+5 M^{2}\right)}{\left(n^{3}+N^{2}\right)^{2}}, \quad t_{2 n}=\frac{2 M_{0}}{\left(n^{2}-1\right)^{2}}, \quad \forall(n, u, v) \in \mathbb{N}_{n_{0}} \times \mathbb{R}^{2} .
\end{gathered}
$$

Clearly (2.1), (2.2) with $i=1,(2.24),(2.86)-(2.88),(2.94)$, and (2.95) hold. Thus, Theorem 2.16 implies that System (3.36) possesses uncountably many bounded positive solutions in $A\left(N, M, N_{0}, M_{0}\right)$.

\section{Acknowledgments}

The authors thank the editor and reviewers for useful comments and suggestions. This study was supported by research funds from Dong-A University.

\section{References}

[1] R. P. Agarwal, Difference Equations and Inequalities, Marcel Dekker, New York, NY, USA, 2nd edition, 2000.

[2] R. P. Agarwal, W.-T. Li, and P. Y. H. Pang, "Asymptotic behavior of nonlinear difference systems," Applied Mathematics and Computation, vol. 140, no. 2-3, pp. 307-316, 2003.

[3] J. Baštinec, L. Berezansky, J. Diblík, and Z. Šmarda, "On the critical case in oscillation for differential equations with a single delay and with several delays," Abstract and Applied Analysis, Article ID 417869, 20 pages, 2010.

[4] J. Baštinec, L. Berezansky, J. Diblík, and Z. Šmarda, "A final result on the oscillation of solutions of the linear discrete delayed equation $\Delta x(n)=-p(n) x(n-k)$ with a positive coefficient," Abstract and Applied Analysis, Article ID 586328, 28 pages, 2011.

[5] J. Baštinec and J. Diblík, "Subdominant positive solutions of the discrete equation $\Delta u(k+n)=$ - $p(k) u(k), "$ Abstract and Applied Analysis, no. 6, pp. 461-470, 2004.

[6] J. Baštinec and J. Diblík, "Remark on positive solutions of discrete equation $\Delta u(k+n)=-p(k) u(k), "$ Nonlinear Analysis, Theory, Methods and Applications, vol. 63, no. 5-7, pp. e2145-e2151, 2005.

[7] J. Baštinec, J. Diblík, and Z. Šmarda, “Existence of positive solutions of discrete linear equations with a single delay," Journal of Difference Equations and Applications, vol. 16, no. 9, pp. 1047-1056, 2010.

[8] J. Baštinec, J. Diblík, and Z. Šmarda, "An Explicit criterion for the existence of positive solutions of the linear delayed equation $\dot{x}(t)=-c(t) x(t-\tau(t))$," Abstract and Applied Analysis, vol. 2011, Article ID 561902, 2011. 
[9] L. Berezansky, J. Diblík, and Z. Šmarda, "Positive solutions of second-order delay differential equations with a damping term," Computers and Mathematics with Applications, vol. 60, no. 5, pp. 13321342, 2010.

[10] J. Diblík and I. Hlavickova, "Asymptotic upper and lower estimates of a class of positive solutions of a discrete linear equation with a single delay," Abstract and Applied Analysis, vol. 2012, Article ID 764351, 12 pages, 2012.

[11] J. Diblík and M. Kúdelčíková, "Two classes of asymptotically different positive solutions of the equation $\dot{y}(t)=-f(t, y(t))$," Nonlinear Analysis. Theory, Methods and Applications A, vol. 70, no. 10, pp. 3702-3714, 2009.

[12] J. Diblík and M. Kúdelčíková, "Existence and asymptotic behavior of positive solutions of functional differential equations of delayed type," Abstract and Applied Analysis, Article ID 754701, 16 pages, 2011.

[13] J. Diblík and Z. Svoboda, "Positive solutions of p-type retarded functional differential equations," Nonlinear Analysis. Theory, Methods and Applications A, vol. 64, no. 8, pp. 1831-1848, 2006.

[14] J. R. Graef and E. Thandapani, "Oscillation of two-dimensional difference systems," Computers and Mathematics with Applications, vol. 38, no. 7-8, pp. 157-165, 1999.

[15] H.-F. Huo and W.-T. Li, "Oscillation of the Emden-Fowler difference systems," Journal of Mathematical Analysis and Applications, vol. 256, no. 2, pp. 478-485, 2001.

[16] H.-F. Huo and W.-T. Li, "Oscillation of certain two-dimensional nonlinear difference systems," Computers and Mathematics with Applications, vol. 45, no. 6-9, pp. 1221-1226, 2003.

[17] J. Jiang and X. Li, "Oscillation and nonoscillation of two-dimensional difference systems," Journal of Computational and Applied Mathematics, vol. 188, no. 1, pp. 77-88, 2006.

[18] J. Jiang and X. Tang, "Oscillation criteria for two-dimensional difference systems of first order linear difference equations," Computers and Mathematics with Applications, vol. 54, no. 6, pp. 808-818, 2007.

[19] J. Jiang and X. Tang, "Oscillation and asymptotic behavior of two-dimensional difference systems," Computers and Mathematics with Applications, vol. 54, no. 9-10, pp. 1240-1249, 2007.

[20] J. Cheng, "Existence of a nonoscillatory solution of a second-order linear neutral difference equation," Applied Mathematics Letters, vol. 20, no. 8, pp. 892-899, 2007.

[21] W.-T. Li, "Classification schemes for nonoscillatory solutions of two-dimensional nonlinear difference systems," Computers and Mathematics with Applications, vol. 42, no. 3-5, pp. 341-355, 2001.

[22] W.-T. Li and S.-S. Cheng, "Remarks on two recent oscillation theorems for second-order linear difference equations," Applied Mathematics Letters, vol. 16, no. 2, pp. 161-163, 2003.

[23] W.-T. Li and S. H. Saker, "Oscillation of second-order sublinear neutral delay difference equations," Applied Mathematics and Computation, vol. 146, no. 2-3, pp. 543-551, 2003.

[24] X. Li and D. Zhu, "New results for the asymptotic behavior of a nonlinear second-order difference equation," Applied Mathematics Letters, vol. 16, no. 5, pp. 627-633, 2003.

[25] H. Liang and P. Weng, "Existence and multiple solutions for a second-order difference boundary value problem via critical point theory," Journal of Mathematical Analysis and Applications, vol. 326, no. 1, pp. 511-520, 2007.

[26] Z. Liu, S. M. Kang, and J. S. Ume, "Existence of uncountably many bounded nonoscillatory solutions and their iterative approximations for second order nonlinear neutral delay difference equations," Applied Mathematics and Computation, vol. 213, no. 2, pp. 554-576, 2009.

[27] Z. Liu, Y. Xu, and S. M. Kang, "Global solvability for a second order nonlinear neutral delay difference equation," Computers and Mathematics with Applications, vol. 57, no. 4, pp. 587-595, 2009.

[28] M. Ma, H. Tang, and W. Luo, "Periodic solutions for nonlinear second-order difference equations," Applied Mathematics and Computation, vol. 184, no. 2, pp. 685-694, 2007.

[29] M. Migda and J. Migda, "Asymptotic properties of solutions of second-order neutral difference equations," Nonlinear Analysis, Theory, Methods and Applications, vol. 63, no. 5-7, pp. e789-799, 2005.

[30] X. H. Tang, "Bounded oscillation of second-order delay difference equations of unstable type," Computers and Mathematics with Applications, vol. 44, no. 8-9, pp. 1147-1156, 2002.

[31] E. Thandapani and P. M. Kumar, "Oscillation of difference systems of the neutral type," Computers and Mathematics with Applications, vol. 54, no. 4, pp. 556-566, 2007.

[32] Y. Tian and W. Ge, "Multiple positive solutions of boundary value problems for second-order discrete equations on the half-line," Journal of Difference Equations and Applications, vol. 12, no. 2, pp. 191-208, 2006.

[33] J. Wu and Y. Liu, "Two periodic solutions of neutral difference systems depending on two parameters," Journal of Computational and Applied Mathematics, vol. 206, no. 2, pp. 713-725, 2007. 
[34] Z. Zhou, J. Yu, and L. Huang, "Asymptotic behavior of delay difference systems," Computers and Mathematics with Applications, vol. 42, no. 3-5, pp. 283-290, 2001.

[35] L. S. Liu, "Ishikawa and Mann iterative process with errors for nonlinear strongly accretive mappings in Banach spaces," Journal of Mathematical Analysis and Applications, vol. 194, no. 1, pp. 114-125, 1995.

[36] Z. G. Zhang and J. S. Yu, "On existence of positive solutions for neutral differential equations," Science in China A, vol. 8, pp. 785-790, 1992 (Chinese). 


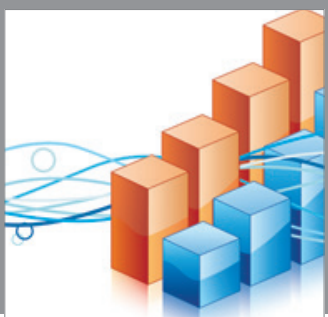

Advances in

Operations Research

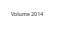

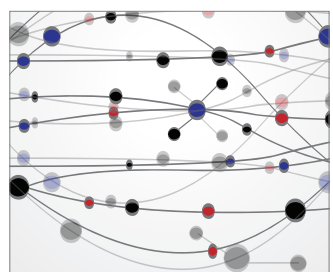

\section{The Scientific} World Journal
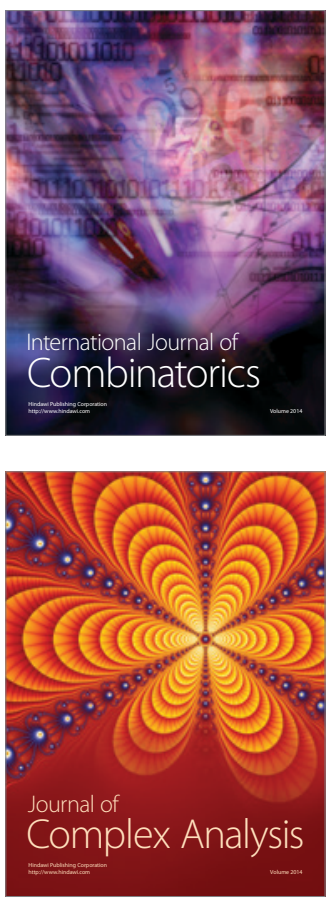

International Journal of

Mathematics and

Mathematical

Sciences
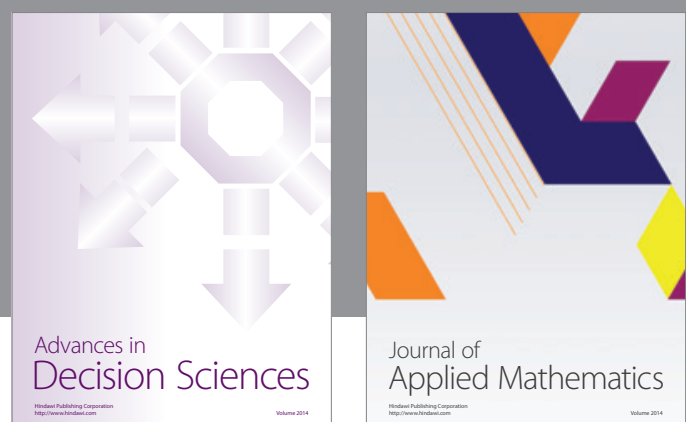

Journal of

Applied Mathematics
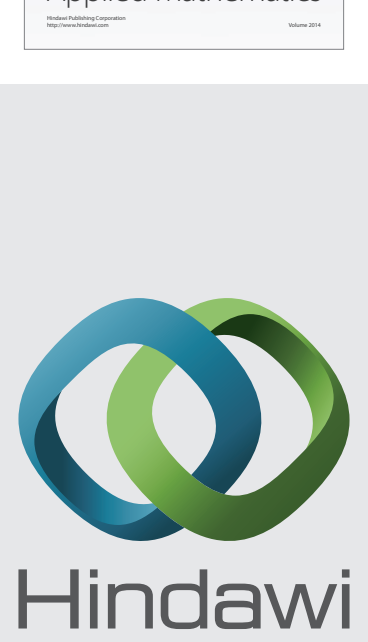

Submit your manuscripts at http://www.hindawi.com
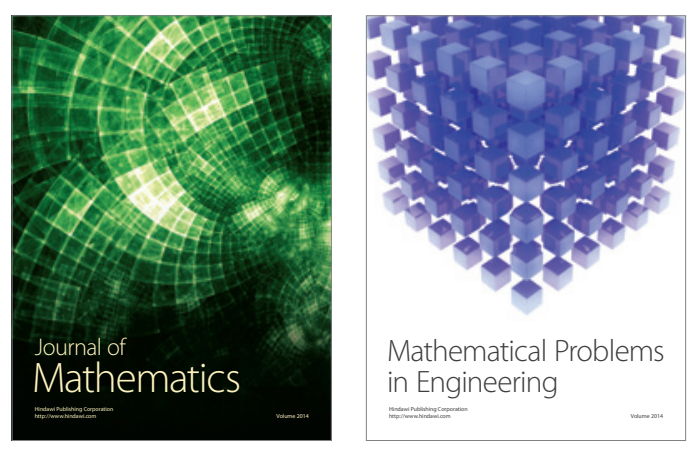

Mathematical Problems in Engineering
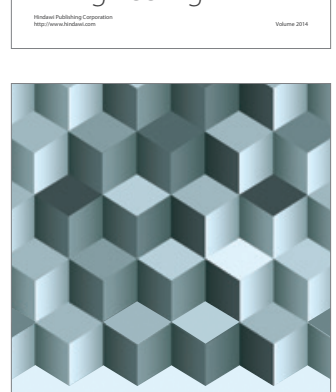

Journal of

Function Spaces
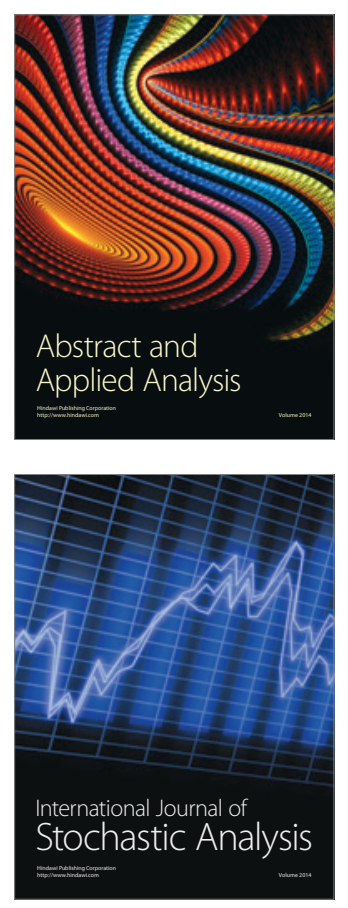

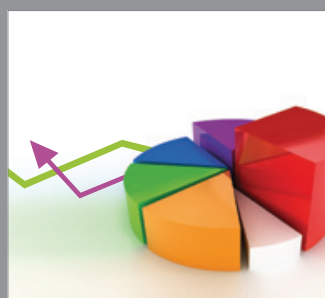

ournal of

Probability and Statistics

Promensencen
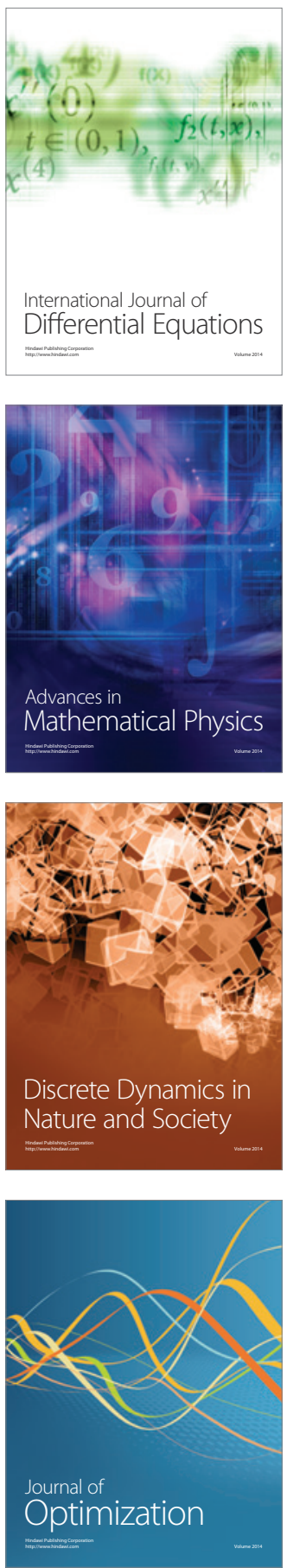